\title{
Órbitas bilhares periódicas em triângulos obtusos
}

\author{
Marisa dos Reis Cantarino
}

\author{
DISSERTAÇÃO APRESENTADA \\ $\mathrm{AO}$ \\ Instituto De Matemática e EstatísticA \\ DA \\ Universidade DE SÃo PAUlo \\ PARA \\ OBTENÇÃO DO TÍTULO \\ $\mathrm{DE}$ \\ Mestre em CIÊNCIAS
}

\author{
Programa: Matemática Aplicada \\ Orientador: Prof. Dr. Manuel Valentim de Pera Garcia
}

Durante o desenvolvimento deste trabalho o autor recebeu auxílio financeiro da CAPES

São Paulo, fevereiro de 2018 


\section{Órbitas bilhares periódicas em triângulos obtusos}

Esta versão da dissertação contém as correções e alterações sugeridas pela Comissão Julgadora durante a defesa da versão original do trabalho, realizada em 09/03/2018. Uma cópia da versão original está disponível no

Instituto de Matemática e Estatística da Universidade de São Paulo.

Comissão Julgadora:

- Prof. Dr. Manuel Valentim de Pera Garcia - IME-USP

- Prof. Dr. Ricardo dos Santos Freire Júnior - IME-USP

- Prof ${ }^{a}$. Dra . Sonia Pinto de Carvalho - ICEx-UFMG 


\section{Agradecimentos}

Meus primeiros agradecimentos são ao meu orientador, Manuel, pela atenção e amizade durante os últimos anos, e por sempre acreditar em mim. Agradeço à Sônia, pelos mesmos motivos e pelo cuidado e ajuda que sempre me concedeu durante a minha vida acadêmica. Obrigada também aos colegas de orientação Matheus, Marcelo, Jerusa e Thiago pela companhia em vários momentos.

Gostaria de agradecer à minha família, família estendida e amigos que foram mais próximos nos últimos dois anos, pelo apoio, carinho e paciência durante a realização deste trabalho: mãe, pai, Marli, Miriam, Marlete, Josi, Ana, Luisa, Rafael, Luiz, Bartira, Luciana, João e Simone. Estando perto ou longe, vocês são fonte de conforto, inspiração e coragem.

Há muitas pessoas que estiveram por perto e me ajudaram a seguir em frente, de uma forma ou de outra. Todo meu carinho a vocês, especialmente para os que compareceram na minha defesa.

Obrigada aos membros da banca pela leitura e considerações sobre este trabalho, assim como à CAPES pelo apoio financeiro sem o qual este não existiria. 


\section{Resumo}

CANTARINO, M. R. Órbitas bilhares periódicas em triângulos obtusos. 2018. 87 f. Dissertação (Mestrado) - Instituto de Matemática e Estatística, Universidade de São Paulo, São Paulo, 2018.

Uma órbita bilhar em um triângulo é uma poligonal cujos segmentos começam e terminam nos lados do triângulo e que se refletem elasticamente nestes lados. É como o movimento de uma bola numa mesa de bilhar sem atrito (logo a bola tem velocidade constante e jamais para) cujas laterais formam um triângulo. Esta órbita é periódica se ela retorna infinitas vezes ao mesmo ponto com a mesma direção.

A existência de órbitas bilhares periódicas em polígonos é uma questão aberta da matemática. Mesmo para um triângulo ainda não há resposta.

Para triângulos agudos, a resposta é bem conhecida, pois o triângulo formato pelos pés das alturas do triângulo é uma órbita periódica. Para triângulos obtusos, em geral, pouco se sabe. O objetivo desta dissertação é coletar resultados e técnicas sobre órbitas bilhares periódicas em triângulos obtusos.

Começamos introduzindo o trabalho de Vorobets, Gal'perin e Stepin, que no início dos anos 90 unificaram os casos conhecidos de triângulos que possuem órbita bilhar periódica, introduziram o conceito de estabilidade e mostraram novos resultados, como uma família infinita de órbitas estáveis. Temos também o teorema de 2000 de Halbeisen e Hungerbühler que estende as famílias de órbitas estáveis.

Mencionamos em seguida os trabalhos de Schwartz de 2006 e 2009 que utilizam auxílio computacional para mostrar que todo triângulo com ângulos menores que $100^{\circ}$ possui órbita bilhar periódica. Depois temos os resultados de 2008 de Hooper e Schwartz sobre órbitas bilhares periódicas em triângulos quase isósceles e sobre estabilidade de órbitas em triângulos de Veech.

Todos os casos abordados neste trabalho incluem uma vasta variedade de triângulos, mas a questão de existência de órbitas bilhares periódicas para todo triângulo está longe de ser totalmente contemplada.

Palavras-chave: dinâmica bilhar, triângulos, órbitas periódicas. 


\section{Abstract}

CANTARINO, M. R. Periodic billiard orbits in obtuse triangles. 2018. 87 p. Thesis (Master's Degree) - Instituto de Matemática e Estatística, Universidade de São Paulo, São Paulo, 2018.

A billiard orbit in a triangle is a polygonal with vertices at the boundary of the triangle such that its angles reflect elastically. It is similar to a moving ball on a billiard table without friction (so the ball has constant speed and never stops) whose sides form a triangle. This orbit is periodic if it returns infinitely to the same point with the same direction.

The existence of periodic billiard orbits in polygons is an open problem in mathematics. Even for a triangle there is still no answer.

For acute triangles the answer is well known since the triangle whose vertices are the base points of the three altitudes of the triangle is a periodic orbit. For obtuse triangles, in general, little is known. The aim of this thesis is to collect results and techniques on periodic billiard orbits in obtuse triangles.

We start by introducing the work of Vorobets, Gal'perin and Stepin, who unified in the early 1990s the known cases of triangles that have periodic billiard orbits, introduced the concept of stability and proved new results, such as an infinite family of stable orbits. We also have the theorem of Halbeisen and Hungerbühler of 2000 extending the families of stable orbits.

Next, we mention the works of Schwartz of 2006 and 2009 that use computational assistance to prove that every triangle whose angles are at most $100^{\circ}$ have periodic billiard orbits. Then, we have the results of 2008 by Hooper and Schwartz on periodic billiard orbits in nearly isosceles triangles and on stability of billiard orbits in Veech triangles.

All cases covered in this work include a wide variety of triangles, but the question of the existence of periodic billiard orbits for all triangles is far from being fully contemplated.

Keywords: billiard dynamics, triangles, periodic orbits. 


\section{Sumário}

$\begin{array}{ll}\text { Introdução } & 1\end{array}$

1 Bilhares em polígonos $\quad \mathbf{5}$

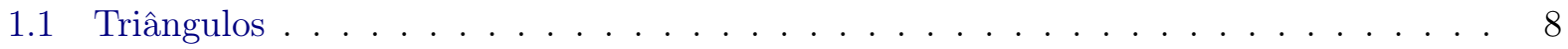

2 Bilhares em triângulos obtusos $\quad 13$

2.1 Órbitas ímpares . . . . . . . . . . . . . . . . . . . . . . . . . 19

2.2 Famílias de órbitas ímpares . . . . . . . . . . . . . . . . . . . . . . 22

2.3 Triângulos obtusos com ângulos menores que $100^{\circ} \ldots \ldots \ldots \ldots$. . . . . . . 27

3 Bilhares em triângulos obtusos quase isósceles 33

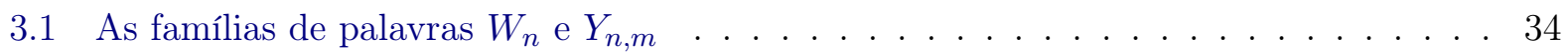

3.2 As famílias de palavras $A_{n}, B_{n}$ e $C_{n} \ldots \ldots \ldots \ldots \ldots$

3.2 .1 Órbita reticulada hexagonal . . . . . . . . . . . . . . . . . . 39

3.2 .2 Órbita reticulada quadrada . . . . . . . . . . . . . . . . . 40

3.2 .3 Funções comparadoras . . . . . . . . . . . . . . . . . . . . . . 41

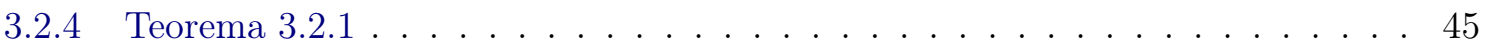

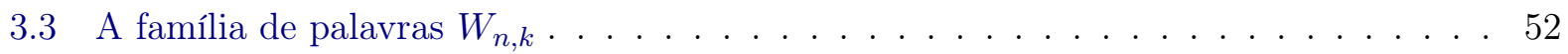

4 Condição homológica para estabilidade de órbitas bilhares em triângulos $\quad 63$

4.1 Órbitas bilhares periódicas em triângulos retângulos são instáveis . . . . . . . . . . . 65

4.2 Estabilidade de órbitas bilhares periódicas em triângulos de Veech . . . . . . . . . . 67

$\begin{array}{ll}\text { A Topologia algébrica } & \mathbf{7 1}\end{array}$

A.1 Homotopia . . . . . . . . . . . . . . . . . . . . 71

A.2 Grupo fundamental . . . . . . . . . . . . . . . . . . . . . . . . . . . . . . .

A.3 Espaços de recobrimento . . . . . . . . . . . . . . . . . . . . . . 74

A.4 Homologia . . . . . . . . . . . . . . . . . . . 75

$\begin{array}{ll}\text { Referências Bibliográficas } & 77\end{array}$

$\begin{array}{ll}\text { Índice Remissivo } & 79\end{array}$ 


\section{Introdução}

A dinâmica de bilhares no plano consiste no fluxo sem atrito de uma partícula sobre um domínio compacto, conexo e com fronteira suave por partes, com velocidade unitária e obedecendo uma reflexão elástica na fronteira deste domínio. Diferentes domínios fornecem diferentes comportamentos de tal sistema.

Mais precisamente, dado um domínio $Y \subset \mathbb{R}^{2}$ compacto, conexo e com fronteira suave por partes, que chamaremos de mesa de bilhar, o fluxo bilhar $T^{t}: \Psi \rightarrow \Psi$ é o movimento com velocidade unitária de um ponto - a "bola de bilhar" — por $Y$, onde o domínio $\Psi$ é o quociente de $Y \times S^{1}$ por uma identificação $(x, v) \sim\left(x, v^{\prime}\right)$. Quando o ponto atinge a fronteira $\partial Y$, a velocidade muda de modo que o componente tangencial se mantém e a componente normal muda de sinal. Como estamos no plano, isso é o mesmo que a lei ótica de reflexão: o ângulo de incidência é igual ao ângulo de reflexão. Chamamos esta reflexão de elástica. Assim, a identificação citada associa $(x, v)$ com $\left(x, v^{\prime}\right)$ se $v^{\prime}$ é a reflexão de $v$ no ponto $x$ da fronteira de $Y$.

Ou seja, o espaço de fase $\Psi$ é o conjunto de todos os vetores tangentes unitários suportados no interior de $Y$ ou suportados em $\partial Y$ que "apontam para dentro". De maneira geral, o fluxo bilhar $T^{t}$ age no fibrado tangente unitário de uma $n$-variedade Riemanniana. O fluxo bilhar são geodésicas no espaço de fase.

Note que esta reflexão não está definida em singularidades da fronteira de $Y$. Se a "bola" atinge o vértice de um polígono, por exemplo, a convenção é que ela para, e não vamos considerar estas órbitas na busca por órbitas periódicas. A seguir, veremos que o conjunto de pontos do espaço de fase tal que a bola atinge um vértice têm medida nula.

A transformação bilhar $\varphi$ é definida pela transformação de Poincaré restrita à seção transversal $\partial Y \times S^{1}$ do espaço de fase. Isto é, a discretização do fluxo para os instantes em que a bola passa pela fronteira de $Y$ :

$$
\begin{aligned}
\varphi: \Phi & \longrightarrow \Phi \\
(x, \theta) & \longmapsto
\end{aligned}
$$

onde $\Phi=\partial Y \times S^{1}$

Ou seja, $\Phi$ é o conjunto de todos os vetores unitários a partir de um ponto de $\partial Y$ que "apontam para dentro" e $\varphi$ é a função que leva o ponto $x$ na direção $\theta$ até chegar na fronteira de $Y$ no ponto $X(x, \theta)$, e então reflete gerando um novo ângulo $\Theta(x, \theta)$.

Se $Y$ tem a fronteira conexa, então $\Psi$ é topologicamente um cilindro preenchido com algumas identificações em sua "lateral". Se $(p, q, \alpha)$ são coordenadas de $\Psi$, com $p$ e $q$ coordenadas cartesianas em $Y$ e $\alpha \in[0,2 \pi)$ é a representação angular dos pontos de $S^{1}$, então temos em $\Psi$ a medida $\nu$ definida pela densidade $d \nu=d p d q d \alpha$. Esta medida é invariante pelo fluxo bilhar.

Sejam $(x, \theta)$ coordenadas em $\Phi$ onde $x$ é parametrizado pelo comprimento $L$ de $\partial Y$, um ponto fixado é 0 e $x \in[0, L]$ é o ponto em sentido anti horário e $\theta$ é o ângulo que o vetor de direção faz com a tangente de $\partial Y$ no ponto $x$. A restrição de $\nu$ para $\Phi$ é $\mu$ definida por $d \mu=\sin \theta d x d \theta$, que é invariante pela transformação bilhar.

Uma órbita bilhar é uma poligonal com vértices na fronteira do domínio cujos ângulos satisfazem a reflexão elástica. Se esta poligonal é fechada, dizemos que a órbita é periódica e dizemos que ela é $n$-segmentada se é composta por $n$ segmentos, com $n \in \mathbb{N}$. Como estamos num fluxo de velocidade constante não nula, órbitas constantes não existem, logo procuramos órbitas periódicas não triviais. 
O caso clássico, introduzido por Birkhoff, é um domínio estritamente convexo com fronteira suave, e neste caso é conhecida a existência de órbitas periódicas. Outro caso estudado é o dispersivo no qual a dinâmica é hiperbólica, ou o semi-dispersivo, e também sempre haverão órbitas periódicas. No caso poligonal, no entanto, ainda não se sabe se sempre existirão órbitas periódicas.

Nos casos convexo e dispersivo, para todo $n \geq 2$ existem limitantes inferiores para a quantidade de órbitas periódicas $n$-segmentadas. Veja [Gut03] para especificidades da dinâmica de bilhares para o caso convexo e dispersivo.

No entanto, para triângulos temos a seguinte:

Proposição 0.0.1. Para todo $n \in \mathbb{N}$ existe um triângulo obtuso $\triangle_{n}$ tal que toda órbita periódica (se existir) possui mais de $n$ segmentos.

Demonstração. Todo triângulo obtuso é definido, a menos de semelhança, por dois ângulos agudos $\alpha$ e $\beta$. Assim, dado $n$ podemos tomar $\alpha$ e $\beta$ tão pequenos de forma que

$$
\min \left\{\left\lceil\frac{\pi}{\alpha}\right\rceil,\left\lceil\frac{\pi}{\beta}\right\rceil\right\}>n
$$

Além disso, vamos exigir que $\alpha, \beta$ e $\pi$ sejam racionalmente independentes, isto é, se $m \alpha+n \beta+$ $l \pi=0 \operatorname{com} l, m, n \in \mathbb{Z}$, então $l=m=n=0$.

Seja $\Gamma$ uma órbita periódica no triângulo obtuso $\triangle_{n}$ com ângulos agudos $\alpha$ e $\beta$. Vamos chamar os lados $\overline{B C}, \overline{A B}$ e $\overline{A C}$ de 1,2 e 3, respectivamente. Vejamos que existe um segmento de $\Gamma$ ligando os lados 1 e 2 .

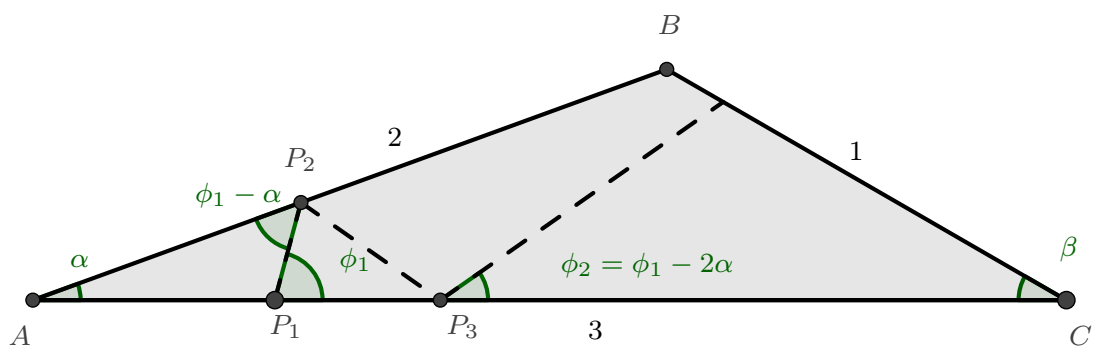

Figura 1: Reflexão quando todos os segmentos da órbita começam ou terminam no lado 3.

Supomos, por absurdo, que não existe um segmento de $\Gamma$ ligando os lados 1 e 2 . Então todo segmento parte do lado 3, reflete em um dos lados 1 ou 2 e retorna ao lado 3. Seja $\phi_{1}$ a inclinação com que a bola de bilhar parte do lado 3 . Veja que na figura 1 ela bate no lado 2 , então reflete com ângulo $\phi_{1}-\alpha$ e retorna ao lado 3 com ângulo $\phi_{2}=\phi_{1}-2 \alpha$. De modo geral, a diferença entre as inclinações $\phi$ e $\psi$ da mesma órbita partindo do lado 3 satisfazem $\phi-\psi=m \alpha+n \beta(\bmod \pi)$ com $m, n \in \mathbb{Z}$.

Como a órbita $\Gamma$ é periódica, a bola de bilhar deve retornar ao ponto inicial com inclinação $\phi$ igual à inclinação inicial $\phi_{0}$. Então $\phi-\phi_{0}+2 k \pi=0, \operatorname{logo} m \alpha+n \beta+2 k \pi=0$. Mas como $\alpha, \beta$ e $\pi$ são racionalmente independentes, então $m=n=k=0$, absurdo.

Agora vejamos que, dado que $\Gamma$ possui um segmento ligando os lados 1 e 2 , então $\Gamma$ possui mais de $n$ segmentos. O segmento entre os lados 1 e 2 tem inclinação $\varepsilon$ com relação ao lado 3 , onde $|\varepsilon|<\min \{\alpha, \beta\}$. Além disso, considerando que a partícula de move de 2 pra 1 , a cada vez que há uma reflexão entre os segmentos que formam o ângulo $\beta$, o ângulo aumenta em $\beta$, como na figura 2 , até que $k \beta+\varepsilon>\frac{\pi}{2}$, e então o ângulo diminui em $\beta$ a cada reflexão até atingir o lado 2. Seja $k(\beta)=\left\lceil\frac{\pi}{\beta}\right\rceil$, como $k(\beta) \beta \geq \pi$, então $\frac{k(\beta)}{2} \beta \geq \frac{\pi}{2}$ se $k(\beta)$ for par e $\frac{k(\beta)+1}{2} \beta>\frac{\pi}{2}$ se $k(\beta)$ for ímpar. Como mesmo acontece entre os lados 2 e 3 , e $n=\min \{k(\alpha), k(\beta)\}$, então $\Gamma$ tem mais de $n$ segmentos. 


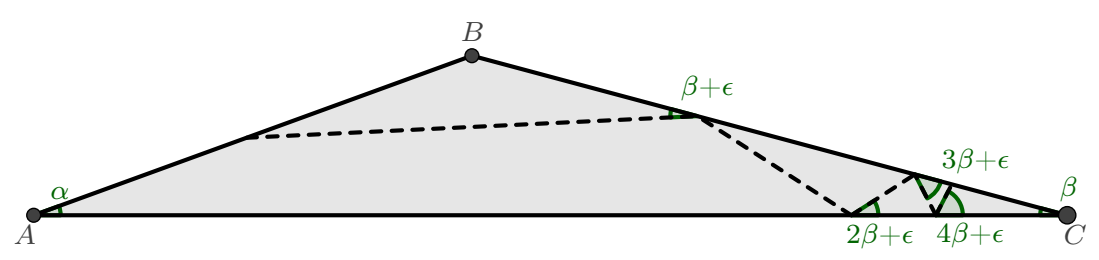

Figura 2: Reflexões entre os lados que formam o ângulo $\beta$.

Para polígonos, mesmo no caso mais simples de um triângulo não há uma abordagem unificada: sabemos que triângulos agudos, isósceles e retângulos possuem órbitas periódicas, assim como alguns casos de triângulos obtusos. Por outro lado, acabamos de ver que existem triângulos cujas menores órbitas periódicas são arbitrariamente longas. Isso reforça que a questão de existência de órbitas periódicas em polígonos é delicada, e esta questão está em aberto até os dias atuais.

Ressaltamos que ao longo desta dissertação adotaremos a convenção de que $\mathbb{N}=\{1,2,3,4, \ldots\}$, isto é, os naturais sem o 0 . Como trabalharemos com quantidade de triângulos ou o número de segmentos de uma órbita, não faz sentido incluir o 0 e deixamos este aviso para evitar a notação $\mathbb{N}^{*}$.

Este trabalho pressupõe do leitor conhecimentos que uma pessoa com graduação em matemática usualmente possui, como o básico de espaços topológicos, grupos ou geometria diferencial, além de conhecimento da linguagem e métodos de sistemas dinâmicos.

No capítulo 1 introduziremos algumas definições, linguagem e técnicas de bilhares em polígonos, assim como mostraremos alguns resultados para polígonos e alguns casos de triângulos.

No capítulo 2 trataremos de alguns resultados específicos para triângulos obtusos e citaremos um teorema (demonstrado com auxílio computacional) que diz que todo triângulo com ângulos menores que $100^{\circ}$ possui órbita bilhar periódica.

Todo o capítulo 3 é dedicado a introduzir definições e resultados auxiliares para provar que toda perturbação suficientemente pequena de um triângulo isósceles tem órbita bilhar periódica. Alguns dos teoremas deste capítulo também foram demonstrados com auxílio computacional.

O capítulo 4 aborda uma condição necessária e suficiente para a estabilidade ${ }^{1}$ de uma órbita bilhar periódica. Damos duas aplicações desta condição: a inexistência de órbitas estáveis em triângulos retângulos e a a inexistência de órbitas estáveis para triângulos obtusos isósceles com ângulos agudos de medida $\frac{\pi}{2^{k+1}}$, com $k=3,4,5, \ldots$.

Incluímos também um apêndice de topologia algébrica, que é usada fundamentalmente no capítulo 4.

Optamos, neste trabalho, por não reproduzir e apenas citar as etapas computacionais das demonstrações auxiliadas por computador. Esta escolha foi feita, dado o tempo finito para realização deste, em favor dos detalhes teóricos que foram priorizados na redação, especialmente do último capítulo e do apêndice.

Para mais detalhes sobre os assuntos abordados nesta introdução, veja [Gut03, Tab95, Tab05, MT02]. As referências principais para os capítulos 1 e 2 são [VGS92, HH00]. Os resultados do capítulo 3 são de [HS09] e do capitulo 4 são de [HS09, Hoo07].

\footnotetext{
${ }^{1}$ Uma órbita bilhar periódica em um polígono é estável se pequenas deformações do polígono não a destroem. Veja a definição no capítulo 2.
} 


\section{Capítulo 1}

\section{Bilhares em polígonos}

A reflexão elástica em um segmento é mais simples do que em uma curva $C^{\infty}$ arbitrária, pois o ângulo de incidência é igual ao ângulo de reflexão com relação ao próprio segmento, e não com relação à sua tangente no ponto, como na figura 1.1 .
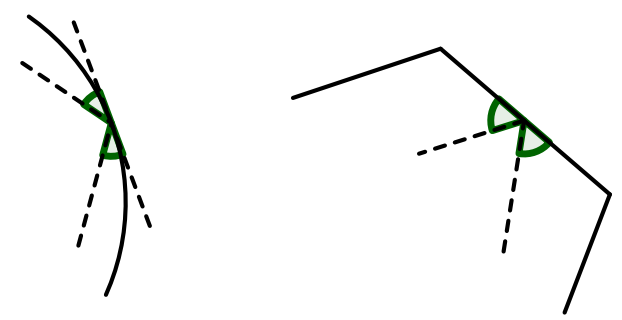

Figura 1.1: À esquerda a reflexão elástica numa fronteira $C^{\infty}$ e à direita a reflexão no lado de um polígono

Isso traz algumas especificidades para o bilhar poligonal, como veremos a seguir. A primeira delas é a técnica de desdobrar a órbita bilhar do polígono: ao invés de realizar a reflexão da trajetória no lado do polígono, mantemos a trajetória reta e refletimos o polígono com relação a este lado.

Definição 1.0.1. Dada uma órbita periódica $\Gamma$ n-segmentada em um polígono $Q$, o desdobramento ${ }^{1}$ de $Q$ com relação a $\Gamma$ é a figura formada por $N(N=n$ se $n$ é par e $N=2 n$ se $n$ é impar $)$ cópias isométricas de $Q$, cada par compartilhando um lado, configuradas do seguinte modo: seja $l_{1} \ldots l_{N}$ a sequência de lados atingidos pela órbita $\Gamma$. Fixamos $Q_{1}=Q$ e $Q_{k}$ é obtido ao refletir $Q_{k-1}$ com relação a reta que passa pelo lado $l_{k-1}$.

Note que se continuássemos as reflexões, teríamos $Q_{N+1}$ como reflexão de $Q_{N}$ com relação ao lado $l_{N}$. Como $\Gamma$ é periódica, então o próximo lado seria $l_{1}$ novamente e estaríamos de volta no ponto de partida com a mesma inclinação. Isso implica que o primeiro e o último lado do desdobramento são paralelos e a órbita periódica liga pontos correspondentes nestes lados em linha reta. Em algumas situações neste trabalho vamos considerar a continuação do desdobramento para algumas demonstrações, mas ficará claro quando isto ocorrer. Veja a figura 1.2.

Exigimos que $N$ seja par para que $Q_{N+1}$ tenha a mesma orientação que $Q$, uma vez que cada reflexão muda a orientação do polígono.

Definição 1.0.2. Dada uma órbita periódica $\Gamma$ em um polígono $Q$, consideramos $\Gamma$ como segmento do desdobramento de $Q$. $O$ feixe de órbitas paralelas associado à $\Gamma$ é composto pelos segmentos paralelos a $\Gamma$ que começam e terminam no primeiro e último lado, estão inteiramente contidos no desdobramento e não passam por nenhum vértice.

\footnotetext{
${ }^{1}$ Do inglês, unfolding.
} 


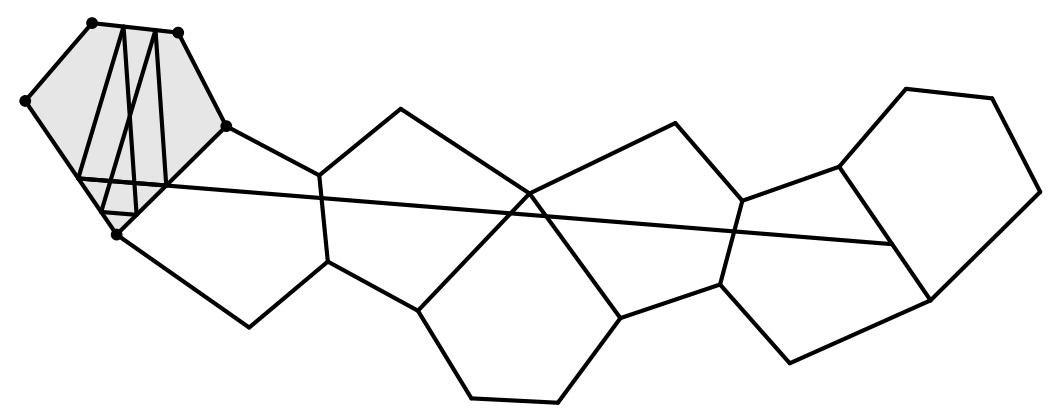

Figura 1.2: Refletimos o polígono com relação ao lado onde a partícula colidiu e sua órbita pode ser representada como um segmento. Se continuarmos a figura, obtemos uma translação do polígono original e a órbita se repete.

Toda órbita com um número par de segmentos faz parte de um feixe de órbitas paralelas, e para toda órbita com um número ímpar de segmentos, realizamos o dobro de reflexões no desdobramento para obter o feixe, como é o caso das órbitas na figura 1.3.
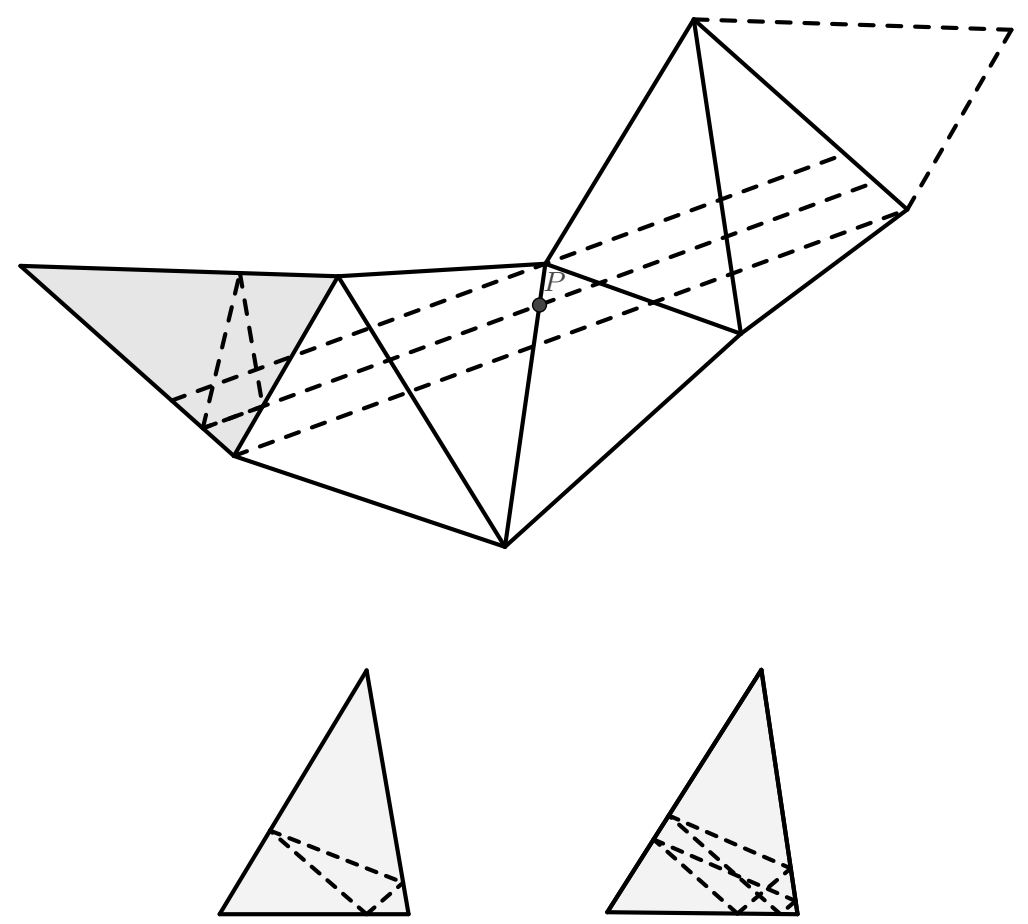

Figura 1.3: Na figura de cima, vemos o desdobramento de uma órbita ímpar e seu feixe de órbitas paralelas. $A$ órbita que passa pelo ponto $P$ é 3-segmentada e as demais órbitas do feixe são 6-segmentadas. Em baixo temos à esquerda uma órbita de 3 segmentos e à direita uma órbita de 6 segmentos paralela à primeira.

Dizemos que $Q$ é um polígono racional se todos os seus ângulos são múltiplos racionais de $\pi$. Um polígono que não é racional é um polígono irracional.

Equivalentemente, um polígono é racional se seu grupo de rotação é finito. De fato, dado um polígono $P$, traçamos retas passando pela origem paralelas a seus lados. Seu grupo de rotação $G(P)$ é o grupo das isometrias lineares do plano geradas por reflexões nestas retas. A composição de duas reflexões é uma rotação e $G(P)$ é um grupo finito se e somente se os ângulos entre estas retas são múltiplos racionais de $\pi$. Quando uma órbita bilhar reflete em um lado de $P$, sua direção muda de acordo com uma ação de $G(P)$.

Mais especificamente, se $P$ é racional e seus ângulos são $\frac{m_{i}}{n_{i}} \pi$ com $m_{i}$ e $n_{i}$ primos entre si, 
tomamos $k=m m c\left\{n_{i}\right\}$. Então $G(P)$ é gerado por reflexões em retas que passam pela origem com ângulos de $\frac{\pi}{k}$ entre si. Assim, $G(P)=D_{k}$, onde $D_{k}$ é o grupo diedral, que é o grupo de simetrias de um polígono regular de $k$ lados e tem $2 k$ elementos, $k$ rotações (uma delas é o elemento neutro) e $k$ reflexões.

O seguinte teorema, por Howard Masur em [Mas86], trata das órbitas bilhares periódicas em polígonos racionais.

Teorema 1.0.1. Todo polígono racional $Q$ possui infinitas órbitas bilhares periódicas. O conjunto formado pelas inclinações de todos os segmentos dessas órbitas é denso em $S^{1}$.

A demonstração deste teorema utiliza teoria de Teichmüller e não fornece um meio de construir tais órbitas. Em [VGS92], Vorobets, Gal'perin e Stepin demonstram um teorema que constrói uma classe de órbitas periódicas em polígonos racionais. As órbitas fornecidas por este teorema são órbitas perpendiculares, isto é, possuem segmentos perpendiculares aos lados de $Q$.

Para mostrar o teorema, antes, demonstremos o seguinte resultado.

Lema 1. Dado um polígono racional $Q$, fixamos um de seus lados horizontalmente e chamamos de lado $\overline{A B}$. Toda órbita bilhar que parte de um ponto $x \in \overline{A B}$ com ângulo $\theta_{0} \in(0, \pi)$ e não atinge um vértice de $Q$ retorna infinitas vezes para uma vizinhança arbitrariamente pequena de $x$ com $o$ mesmo ângulo $\theta_{0}$.

Demonstração. Consideramos o espaço de fase do fluxo bilhar $Q \times S^{1}$. Para cada $\theta \in S^{1}$, o fluxo no interior de $Q \times\{\theta\}$ é o movimento constante na direção $\theta$. Para os pontos $x$ de $\partial Q \times\{\theta\}$, fazemos a identificação $(x, \theta) \sim\left(x, \theta^{\prime}\right)$, onde $\theta$ e $\theta^{\prime}$ são ângulos de incidência e reflexão no lado ao qual $x$ pertence.

Então, o movimento do ponto $\left(x, \theta_{0}\right) \in Q \times S^{1}$ é o movimento constante no interior de $Q_{0}=$ $Q \times\left\{\theta_{0}\right\}$, até atingir a borda, onde o ponto "muda de andar" no espaço de fase, passando a percorrer o interior do "andar" $Q_{1}=Q \times\left\{\theta_{0}^{\prime}\right\}$ em linha reta. Como o polígono $Q$ é racional, o ângulo inicial $\theta_{0}$ muda com a ação de um grupo finito a cada reflexão, logo o ponto se move por um número finito de "andares" $Q_{0}, Q_{1}, \ldots, Q_{k}$.

Ainda, como a transformação bilhar $\varphi$ preserva a medida $\mu$ e o espaço de fase $\partial Q \times S^{1}$ tem medida finita, podemos aplicar o teorema da recorrência de Poincaré na transformação bilhar: se $U \subset \partial Q \times S^{1}$ é mensurável e tem medida positiva, para quase todo ponto $p \in U$ existem infinitos $n \in \mathbb{N}$ tais que $\varphi^{n}(p) \in U$. Em outras palavras, todo ponto retorna infinitas vezes arbitrariamente próximo do local de partida.

Tomamos então conjuntos mensuráveis da forma ${ }^{2} U_{\varepsilon}=\left\{(y, \theta): d(x, y)<\varepsilon\right.$ e $\left.d\left(\theta_{0}, \theta\right)<\varepsilon\right\}$. Seja $d_{i j}=\left|\theta_{i}-\theta_{j}\right|$, onde $\theta_{i}$ é o ângulo do ponto no conjunto $Q_{i}$. Tomamos $\varepsilon=\min _{d_{i j} \neq 0}\left\{d_{i j}\right\}$. Então quase todo ponto de $U_{\varepsilon}$ retorna infinitas vezes para $U_{\varepsilon}$. Pela escolha de $\varepsilon$, este ponto retorna a uma distância menor que $\varepsilon$ de $x$ e com ângulo exatamente $\theta_{0}$, pois nenhum $Q_{i}$ está a uma distância menor que $\varepsilon$ de $Q_{0}$.

Como podemos aplicar o teorema de recorrência para todo $U_{\varepsilon^{\prime}}$ com $0<\varepsilon^{\prime}<\varepsilon$, segue que a órbita de $\left(x, \theta_{0}\right)$ retorna infinitas vezes para um ponto próximo de $x$ com ângulo $\theta_{0}$.

Teorema 1.0.2. Todo n-gono racional $Q$ contém pelo menos $\frac{n}{2}$ feixes de órbitas periódicas perpendiculares.

Demonstração. Fixamos um lado de $Q$ horizontalmente e o chamamos de lado $\overline{A B}$. Tomamos um ponto $x \in \overline{A B}$ tal que sua órbita bilhar não atinge um vértice. Em seguida, lançamos o ponto perpendicularmente ao lado $\overline{A B}$, isto é, consideramos a órbita de $\left(x, \frac{\pi}{2}\right)$. Vejamos que esta órbita é periódica.

Aplicamos o lema $1 \operatorname{com} \theta_{0}=\frac{\pi}{2}$. Então a órbita retorna infinitas vezes próxima do ponto $\left(x, \frac{\pi}{2}\right)$. Como na reflexão em um polígono com ângulo de incidência $\frac{\pi}{2}$ o ângulo de reflexão também é $\frac{\pi}{2}$, a

\footnotetext{
${ }^{2}$ Aqui a distância em $Q$ é euclideana e a distância em $S^{1}$ é a diferença entre os ângulos em $\mathbb{R}$.
} 
órbita retorna pelo mesmo caminho que veio até atingir o ponto $\left(x, \frac{\pi}{2}\right)$ novamente. Logo, a órbita é periódica.

Para cada lado que fixamos horizontalmente, contamos um feixe (claro que podem haver mais). Como uma órbita pode ser perpendicular a, no máximo, 2 lados, então podemos estar contando o mesmo feixe duas vezes. Logo, temos pelo menos $\frac{n}{2}$ feixes de órbitas bilhares perpendiculares.

\subsection{Triângulos}

Em um triângulo agudo, o triângulo inscrito que conecta os pés das 3 alturas, chamado triângulo de Fagnano, é uma órbita bilhar periódica. De fato, dado um triângulo agudo $A B C$, sejam $P, Q$ e $R$ os pés das alturas que passam por $C, B$ e $A$, respectivamente. Queremos mostrar que os ângulos de reflexão são iguais aos ângulos de incidência, isto é, que $B \hat{R} P=C \hat{R} Q, B \hat{P} R=A \hat{P} Q$ e $A \hat{Q} P=C \hat{Q} R$. Vejamos que $B \hat{R} P=C \hat{R} Q$, os demais casos são análogos.

Os triângulos $B P O$ e $B R O$ são ambos retângulos com o lado oposto ao ângulo reto tendo o mesmo comprimento. Como um triângulo retângulo está sempre inscrito num semicírculo cujo diâmetro é seu maior lado, o quadrilátero BROP está inscrito numa circunferência. Os ângulos $P \hat{B} O$ e $P \hat{R} O$ são iguais pois $B$ e $R$ estão na circunferência e os ângulos estão suportados no mesmo arco. Do mesmo modo, vemos que $Q \hat{C} O=Q \hat{R} O$. Como o triângulo $A P C$ é retângulo, $B \hat{A} C=P \hat{A} C=\pi-Q \hat{C} O$, e como $A Q B$ é um triângulo retângulo, $B \hat{A} C=B \hat{A} Q=\pi-P \hat{B} O$. Logo, $Q \hat{C} O=P \hat{B} O$, o que implica que $P \hat{R} O=Q \hat{R} O$ e, consequentemente, $B \hat{R} P=C \hat{R} Q$.

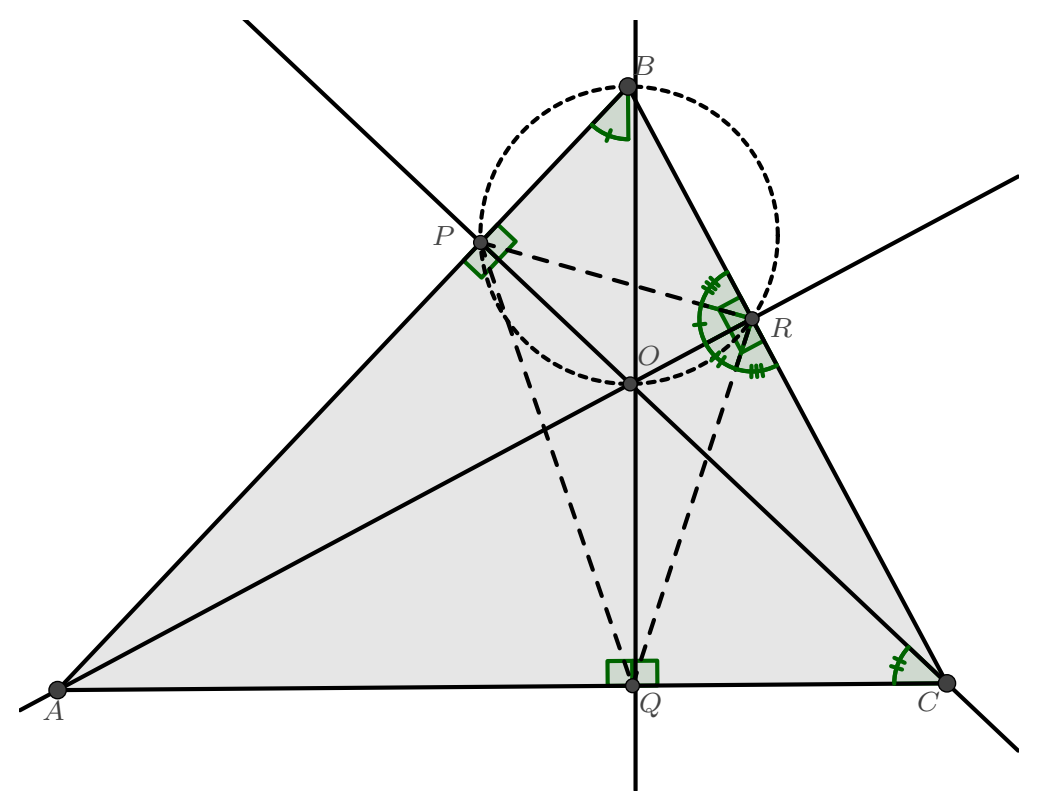

Figura 1.4: O triângulo de Fagnano de fato é uma órbita bilhar periódica.

Esta órbita é chamada de órbita bilhar de Fagnano.

Para triângulos que satisfazem uma relação especial entre os ângulos, em particular, triângulos isósceles e retângulos, temos o resultado a seguir.

Teorema 1.1.1. Dado um triângulo com ângulos agudos $\alpha$ e $\beta$ que satisfazem $k \alpha=l \beta<\frac{\pi}{2}$, com $k, l \in \mathbb{N}$. Então este triângulo possui uma órbita periódica perpendicular $2(k+l)$-segmentada.

Demonstração. Fixamos o maior lado do triângulo horizontalmente e nomeamos os vértices desta base de $A$ e $B$, da esquerda pra direita. O vértice $C$ é o que está oposto ao maior lado. Sem perda de generalidade, chamamos o ângulo do vértice $A$ de $\alpha$ e o ângulo do vértice $B$ de $\beta$. Fazemos $k-1$ reflexões do triângulo com relação ao lado $\overline{A C}$ de modo que elas tem o vértice $A$ em comum. 
Fazemos $l$ reflexões do triângulo com relação ao lado $\overline{A B}$ de modo que elas tem o vértice $B$ em comum. Veja figura 1.5.

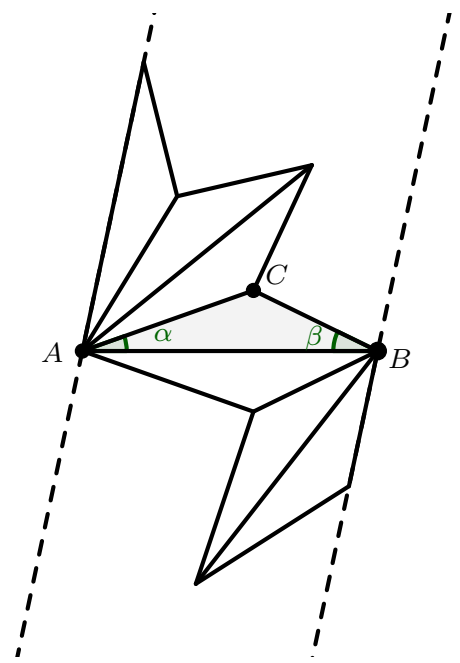

Figura 1.5: A condição $k \alpha=l \beta<\frac{\pi}{2}$ garante que dois lados do desdobramento sejam paralelos

Como $k \alpha=l \beta<\frac{\pi}{2}$ o ângulo agudo que a última aresta à esquerda deste desdobramento faz com a horizontal $(k \alpha)$ é igual ao ângulo agudo que a última aresta à direita faz com a horizontal $(l \beta)$. Logo, as retas tracejadas da figura 1.5 são paralelas. Além disso, como $k \alpha=l \beta$ é agudo, podemos traçar um seguimento perpendicular a estas retas que está contido no desdobramento e não passa por nenhum vértice. Este segmento é uma órbita periódica. Como é uma órbita perpendicular, ela parte de um dos lados extremos do desdobramento, percorre até o outro e volta pelo mesmo caminho, logo ela é $2(k+l)$-segmentada.

Corolário 1. Fazendo $k=l=1$, obtemos que triângulos isósceles possuem órbita periódica. Como um triângulo retângulo pode ser desdobrado em um triângulo isósceles, também há órbitas periódicas em um triângulo retângulo, como na figura 1.6.
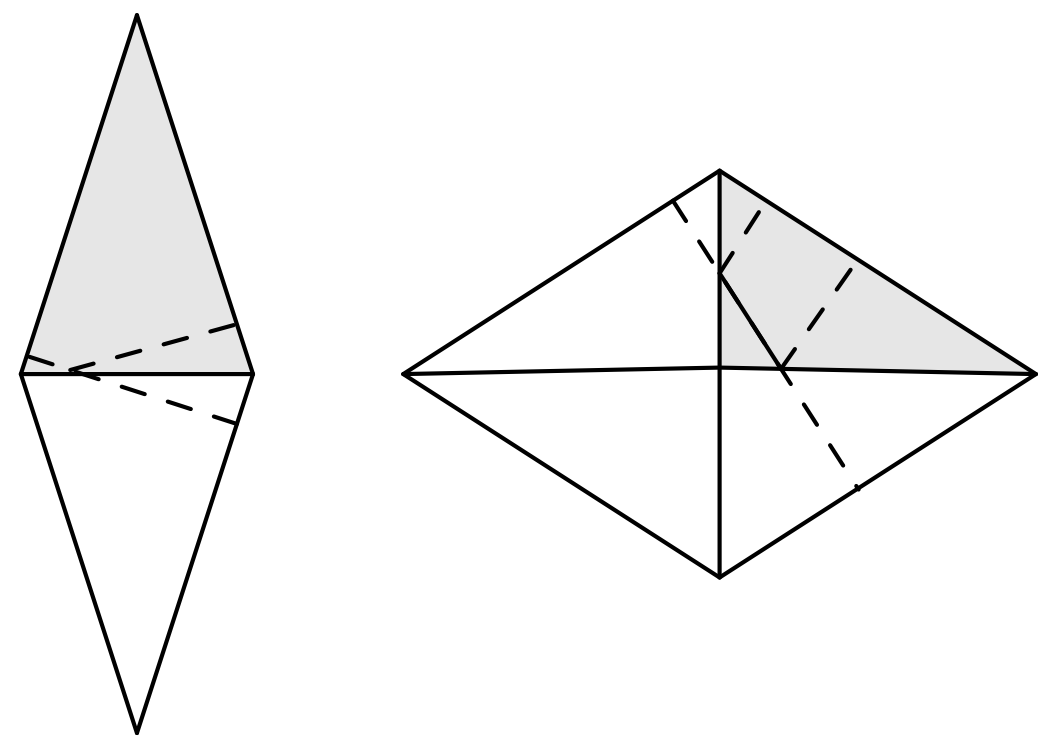

Figura 1.6: Corolário 1

Outro resultado, demonstrado em [HH00], é o 


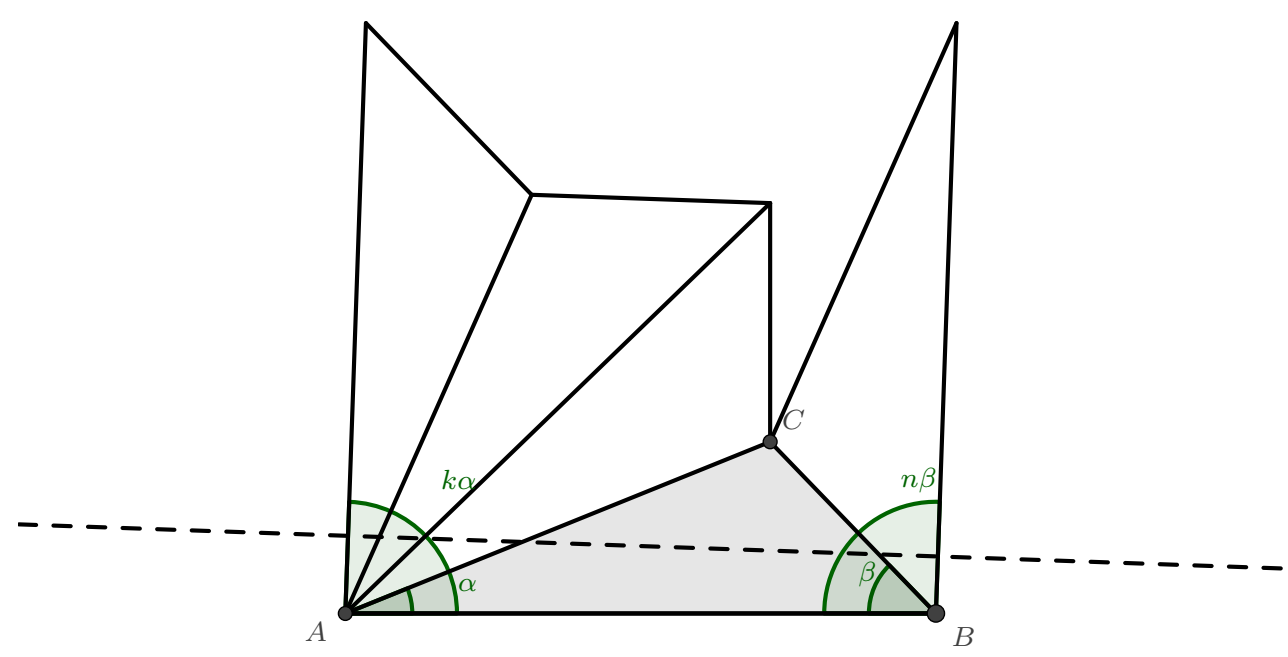

Figura 1.7: Ilustração do caso $k \alpha<\frac{\pi}{2}$.

Teorema 1.1.2. Seja $\triangle A B C$ com ângulos agudos $\alpha$ e $\beta$ que satisfazem $k \alpha+n \beta=\pi$, com $k, n \in \mathbb{N}$ $e$

$$
\frac{\pi}{2 k}\left(1-\frac{1}{n-1}\right)<\alpha<\frac{\pi}{2 k}\left(1+\frac{1}{k-1}\right)
$$

desconsiderando o lado direito ou esquerdo da desigualdade se $n=1$ ou $k=1$. Então $\triangle A B C$ contém um feixe de órbitas paralelas $2(n+k-1)$-segmentadas.

Demonstração. Fixamos $\overline{A B}$ horizontalmente e consideramos o desdobramento do triângulo de modo que temos $n-1$ reflexões a partir do lado $\overline{B C}$ em torno do ponto $B$ e $k-1$ reflexões a partir do lado $\overline{A C}$ em torno do ponto $A$. Como $k \alpha+n \beta=\pi$, então o primeiro e o último lado do desdobramento são paralelos. Logo, falta verificar que a condição

$$
\frac{\pi}{2 k}\left(1-\frac{1}{n-1}\right)<\alpha<\frac{\pi}{2 k}\left(1+\frac{1}{k-1}\right)
$$

é suficiente para que exista uma órbita periódica neste desdobramento.

Note que se $k \alpha<\frac{\pi}{2}$, a extensão do segmento definido pela órbita desdobrada (tracejado na figura 1.7) intercepta a reta $\overleftrightarrow{A B}$ num ponto mais próximo de $B$, e se $k \alpha>\frac{\pi}{2}$, esta extensão intercepta a mesma reta mais próximo de $A$. No primeiro caso, o segmento perpendicular ao primeiro e ao último lado do desdobramento tem inclinação $\frac{\pi}{2}-k \alpha$ e no segundo caso, a inclinação é $\frac{\pi}{2}-n \beta$. Para que o segmento tracejado seja um órbita periódica perpendicular, basta que ele intercepte $\overline{A C}$ e $\overline{B C}$, assim, é suficiente que

- Caso 1: $\frac{\pi}{2}-k \alpha<\beta$;

- Caso $2: \frac{\pi}{2}-n \beta<\alpha$. 


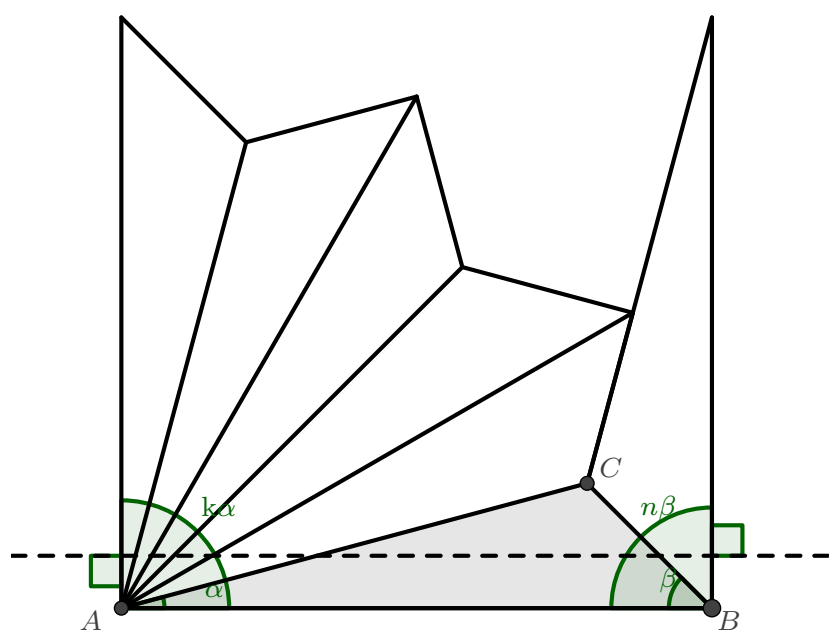

Figura 1.8: No caso $k \alpha=\frac{\pi}{2}$, a órbita periódica é paralela ao lado $\overline{A B}$.

Mas as duas desigualdades acima são equivalentes a condição 1.1. De fato

$$
\begin{aligned}
\frac{\pi}{2 k}\left(1-\frac{1}{n-1}\right) & <\alpha \Longleftrightarrow \frac{\pi}{2 k}\left(\frac{n-2}{n-1}\right)<\alpha \Longleftrightarrow(n-2) \pi<2 k \alpha(n-1) \\
& \Longleftrightarrow \frac{n \pi-2 n k \alpha}{2 n}<\frac{2 \pi-2 k \alpha}{2 n} \Longleftrightarrow \frac{\pi}{2}-k \alpha<\frac{\pi-k \alpha}{n}=\beta
\end{aligned}
$$

e, do mesmo modo,

$$
\begin{aligned}
\alpha & <\frac{\pi}{2 k}\left(1+\frac{1}{k-1}\right) \Longleftrightarrow \alpha<\frac{\pi}{2 k}\left(\frac{k}{k-1}\right) \\
& \Longleftrightarrow(k-2) \pi<2 \pi(\pi-k \alpha)(k-1) \Longleftrightarrow(k-2) \pi<2 n \beta(k-1) \Longleftrightarrow \frac{\pi}{2}-n \beta<\alpha
\end{aligned}
$$

O caso $k \alpha=\frac{\pi}{2}$ pode ser visto na figura 1.8 .

O corolário a seguir aparece independentemente em [VGS92]. Basta tomar $n=2$ e $k$ múltiplo de 2 .

Corolário 2. Um triângulo obtuso cujos ângulos agudos satisfazem ${ }^{3} k \alpha+\beta=\frac{\pi}{2}$, com $k \in \mathbb{N} e$ $2 \beta+\alpha>\frac{\pi}{2}$ possui órbita bilhar periódica perpendicular.

Corolário 3. Se o triângulo for retângulo, temos $k=n=2$ em $k \alpha+n \beta=\pi$ no teorema 1.1.2.

Este é o mesmo caso do corolário 1, que também foi demonstrado independentemente por Fred Holt em [Hol93].

\footnotetext{
${ }^{3}$ Vorobets, Gal'perin e Stepin chamam os triângulos que satisfazem a condição deste corolário de triângulos quase retângulos, pois, se $k=1$, temos um triângulo retângulo.
} 


\section{Capítulo 2}

\section{Bilhares em triângulos obtusos}

Neste capítulo, focaremos em técnicas e resultados para órbitas bilhares em triângulos obtusos. No entanto, alguns resultados valem em polígonos quaisquer e nestes casos enunciaremos os resultados de maneira mais geral.

Dado um triângulo, podemos representá-lo no quadrado aberto $\left(0, \frac{\pi}{2}\right) \times\left(0, \frac{\pi}{2}\right)$, onde o par $(\alpha, \beta)$ representa dois ângulos agudos do triângulo. Se tratando de órbitas bilhares, os tamanhos dos lados não importam, então esta representação preserva a única característica importante do triângulo, que é sua forma. Esta é determinada pelos ângulos a menos de semelhança.

É claro que esta representação não é única. Um triângulo agudo de ângulos $\frac{2}{9} \pi, \frac{1}{3} \pi$ e $\frac{4}{9} \pi$, por exemplo, pode ser representado pelos pontos $\left(\frac{2}{9} \pi, \frac{1}{3} \pi\right),\left(\frac{1}{3} \pi, \frac{2}{9} \pi\right),\left(\frac{2}{9} \pi, \frac{4}{9} \pi\right),\left(\frac{4}{9} \pi, \frac{2}{9} \pi\right)$, $\left(\frac{1}{3} \pi, \frac{4}{9} \pi\right)$ ou $\left(\frac{4}{9} \pi, \frac{1}{3} \pi\right)$. Os triângulos obtusos, por outro lado, possuem apenas dois ângulos agudos, então um triângulo obtuso de ângulos $\frac{1}{4} \pi, \frac{1}{6} \pi$ e $\frac{7}{12} \pi$, por exemplo, pode ser representado pelos pontos $\left(\frac{1}{4} \pi, \frac{1}{6} \pi\right)$ ou $\left(\frac{1}{6} \pi, \frac{1}{4} \pi\right)$.

O caso dos triângulos agudos já foi considerado no capítulo 1, então vamos restringir o espaço dos parâmetros a $\Delta=\left\{(\alpha, \beta): 0<\alpha, 0<\beta, \alpha+\beta<\frac{\pi}{2}\right\}$ na maioria dos casos considerados a partir daqui. Note que, se denotarmos o maior lado por lado 3 e, em sentido anti-horário, os demais lados por 1 e 2 , e chamarmos o ângulo entre os lados 2 e 3 de $\alpha$ e o ângulo entre os lados 1 e 3 de $\beta$, teremos entre este espaço de parâmetros e o conjunto de todos os triângulos obtusos uma bijeção, a menos de rotação, translação ou homotetia. Denotaremos por $T_{(\alpha, \beta)}$ o elemento do quociente de todos os triângulos pela relação de equivalência que associa triângulos que podem ser obtidos via transformações que preservam orientação e ângulos, ou seja, dois triângulos são equivalentes se são semelhantes com a mesma orientação. Assim distinguimos, para $\alpha \neq \beta$, o triângulo $T_{(\alpha, \beta)}$ do triângulo $T_{(\beta, \alpha)}$ : um é a reflexão do outro, portanto têm orientações opostas.

Com isso, vamos identificar a cada ponto $(\alpha, \beta) \in \Delta$ com um triângulo obtuso $T_{(\alpha, \beta)}$ e, muitas vezes, cometeremos um abuso de linguagem e diremos "o triângulo $(\alpha, \beta)$ ".

Feito isso, podemos considerar no conjunto dos triângulos obtusos todos os triângulos que sabemos possuir órbita periódica. Munimos o espaço dos parâmetros com a topologia induzida de $\mathbb{R}^{2}$ e podemos nos perguntar:

1. Dado um triângulo e uma órbita periódica nele, existe uma vizinhança deste triângulo tal que, para todo ponto desta vizinhança, a órbita "se mantém" refletindo nos mesmos lados?

2. É possível cobrir o espaço dos parâmetros por uma família de abertos definidos como vizinhanças de triângulos onde todos possuem órbita periódica?

Não se sabe a resposta da pergunta (2), mas uma resposta afirmativa resolveria a questão de existência de órbitas periódicas para todos os triângulos. Chamaremos as órbitas dos triângulos 
para os quais a pergunta (1) tem resposta afirmativa de estáveis. Este conceito pode ser definido para um polígono arbitrário.

Definição 2.0.1. Uma órbita periódica num polígono $Q$ é uma órbita estável se com "pequenas deformações" do polígono a órbita "continua existindo", refletindo-se na mesma sequência de lados.

No caso dos triângulos, com as notações introduzidas acima, podemos fazer esta definição mais precisa dizendo que uma órbita periódica $\Gamma$ num triângulo $T_{(\alpha, \beta)}$ é estável se existe $U \subseteq \Delta$ aberto tal que $(\alpha, \beta) \in U$ e para todo $T \in U$ existe uma órbita $\Gamma^{\prime}$ em $T$ refletindo-se na mesma sequência de lados que $\Gamma$. Uma órbita instável é uma órbita não estável.

Podemos codificar as órbitas em triângulos usando palavras num alfabeto de três símbolos, onde cada símbolo corresponde a um lado. Mais geralmente, podemos codificar órbitas em um $n$ gono usando palavras num alfabeto de $n$ símbolos. Com isto, obtemos uma condição necessária e suficiente para a estabilidade de uma órbita apenas com base nessas palavras e no desdobramento que elas geram.

Vamos fixar nosso alfabeto como $\{1,2,3\}$. Uma palavra finita de comprimento $k \in \mathbb{N}$ neste alfabeto é um elemento de $\{1,2,3\}^{k}$. Uma palavra infinita é um elemento de $\{1,2,3\}^{\mathbb{N}}$ e uma palavra bi-infinita é um elemento de $\{1,2,3\}^{\mathbb{Z}}$. Podemos representar estas palavras respectivamente como $\omega=\omega_{1} \ldots \omega_{k}, \omega=\omega_{1}, \omega_{2}, \ldots$ e $\omega=\ldots \omega_{-1}, \omega_{0}, \omega_{1}, \ldots$ É claro que nem todas as palavras representam uma órbita bilhar periódica em algum triângulo. Por exemplo, as palavras com símbolos consecutivos repetidos $\left(\omega_{j}=\omega_{j+1}\right.$ para algum $\left.j\right)$ não podem representar órbitas bilhares periódicas pois a bola, percorrendo segmentos de reta em um polígono, não pode partir de um lado e chegar logo em seguida a ele mesmo.

Para cada palavra finita $\omega$ de comprimento $k$, podemos definir sua continuação periódica como a repetição infinita de seus elementos na mesma ordem $\bar{\omega}=\omega_{1} \ldots \omega_{k} \omega_{1} \ldots \omega_{k} \ldots$ Do mesmo modo, a continuação periódica bi-infinita é a palavra $\hat{\omega}=\ldots \omega_{1} \ldots \omega_{k} \omega_{1} \ldots \omega_{k} \ldots$, com repetição de seus elementos de modo que $i \equiv j \bmod n$ implica $\hat{\omega}_{i}=\hat{\omega}_{j}$.

Duas palavras são equivalentes se suas continuações periódicas bi-infinitas são iguais a menos de translação dos símbolos. Por exemplo,

$$
\omega_{1} \ldots \omega_{k-2} \omega_{k-1} \omega_{k} \sim \omega_{k} \omega_{1} \ldots \omega_{k-2} \omega_{k-1} \sim \omega_{k-1} \omega_{k} \omega_{1} \ldots \omega_{k-2} .
$$

Outra maneira de pensar em palavras finitas equivalentes é dispor seus símbolos em círculo. Se uma pode ser obtida com uma rotação da outra, elas são equivalentes.

Dada uma órbita $\Gamma$, definimos $\omega(\Gamma)$ como a palavra associada a $\Gamma$, determinada pela sequência de lados que $\Gamma$ atinge. Se $\Gamma$ é aperiódica, então $\omega(\Gamma)$ é uma palavra bi-infinita. Se $\Gamma$ é periódica e $n$-segmentada, então $\omega(\Gamma)$ é uma palavra bi-infinita de período $n$ e vamos representá-la por uma palavra finita equivalente de comprimento $N$, com $N=n$ se $n$ é par e $N=2 n$ se $n$ é impar. Note que não temos uma única $\omega(\Gamma)$ para cada $\Gamma$ periódica. Para cada lado que fixamos como o início da órbita, obtemos uma palavra diferente, porém elas são sempre equivalentes.

No capítulo 1, definimos em 1.0.1 o desdobramento de uma órbita em um polígono. Agora que temos uma correspondência entre órbitas e palavras, podemos definir o desdobramento de um polígono com relação a uma palavra.

Definição 2.0.2. Dada uma palavra de comprimento $N$, com $N$ par, o desdobramento de um polígono $Q$ com relação a palavra $\omega$, denotado por $U(\omega, Q)$ é a figura formada por $N$ cópias isométricas de $Q$, cada par compartilhando um lado, de modo que $Q_{1}=Q$ e $Q_{k}$ é obtido ao refletir $Q_{k-1}$ com relação ao lado $\omega_{k-1}$.

Definição 2.0.3. Uma palavra finita $\omega$ de comprimento $n$, com $n$ par, é uma palavra estável se cada símbolo aparece o mesmo número de vezes em posição par e em posição ímpar.

Note que a estabilidade de uma palavra é invariante por equivalência de palavras. A terminologia usada na definição 2.0.3 é motivada pelo seguinte resultado. 
Teorema 2.0.1. A órbita $\Gamma$ no polígono $Q$ é estável se, e somente se, a palavra $\omega(\Gamma)$ é estável e o desdobramento $U(\omega(\Gamma), Q)$ contém um segmento ligando o primeiro e o último lado em pontos correspondentes que não intercepta nenhum vértice.

Demonstração. Seja $k \in \mathbb{N}$ o número de lados de $Q$. Nomeamos cada lado como $1,2, \ldots k$. Fixaremos também que $N=n$ se $n$ é par e $N=2 n$ se $n$ é ímpar, onde $n$ é o número de segmentos de $\Gamma$.

$(\Rightarrow)$ Consideramos o desdobramento de $Q$ com relação a $\Gamma$. Como $\Gamma$ é periódica, $Q_{N}$ é paralelo a $Q$.

Fixadas coordenadas cartesianas no plano, consideramos os ângulos que cada lado de $Q$ forma com a horizontal: $\alpha_{i}$ é o ângulo do lado $i, i=1, \ldots, k$. Então refletir o polígono com relação ao lado $i$ é refletir com relação à reta de inclinação $\alpha_{i}$ passando pela origem e fazer uma translação. A composição de duas reflexões, uma com ângulo $x$ e outra com ângulo $y$, nos dá uma rotação de ângulo $x-y$.

De fato,

$$
\left(\begin{array}{cc}
\cos x & \sin x \\
\sin x & -\cos x
\end{array}\right) \times\left(\begin{array}{cc}
\cos y & \sin y \\
\sin y & -\cos y
\end{array}\right)=\left(\begin{array}{cc}
\cos (x-y) & -\sin (x-y) \\
\sin (x-y) & \cos (x-y)
\end{array}\right) .
$$

Logo, se $\omega_{1}, \ldots, \omega_{N}$ é a sequência de lados que $\Gamma$ percorre, a rotação do polígono $Q$ para obter o polígono $Q_{N}$ tem ângulo

$$
\left(\alpha_{\omega_{1}}-\alpha_{\omega_{2}}\right)+\cdots+\left(\alpha_{\omega_{N-1}}-\alpha_{\omega_{N}}\right)=2 m \pi
$$

que é múltiplo de $2 \pi$ pois $Q_{N}$ é paralelo a $Q$.

Mas, da estabilidade de $Q$, para perturbações suficientemente pequenas $\delta_{1}, \ldots, \delta_{k}$ dos ângulos para obter um polígono $Q^{\prime}$, a igualdada acima é mantida

$$
\left(\alpha_{\omega_{1}}+\delta_{\omega_{1}}-\alpha_{\omega_{2}}-\delta_{\omega_{2}}\right)+\cdots+\left(\alpha_{\omega_{N-1}}+\delta_{\omega_{N-1}}-\alpha_{\omega_{N}}-\delta_{\omega_{N}}\right)=2 m \pi,
$$

$\operatorname{logo} \delta_{\omega_{1}}-\delta_{\omega_{2}}+\cdots+\delta_{\omega_{N-1}}-\delta_{\omega_{N}}=0$. Como isto deve valer para $\delta_{1}, \ldots, \delta_{k}$ arbitrários, então a palavra $w(\Gamma)=\omega_{1} \ldots \omega_{N}$ é estável.

$(\Leftarrow)$ Se $\omega(\Gamma)$ é estável, então o primeiro e o último lado do desdobramento $U(\omega(\Gamma), Q)$ são paralelos, uma vez que sempre temos

$$
\left(\alpha_{\omega_{1}}-\alpha_{\omega_{2}}\right)+\cdots+\left(\alpha_{\omega_{N-1}}-\alpha_{\omega_{N}}\right)=0 .
$$

Logo, se $U(\omega(\Gamma), Q)$ contém um segmento ligando o primeiro e o último lado em pontos correspondentes que não intercepta nenhum vértice, este segmento é uma órbita periódica. Além disso, com perturbações $\delta_{1}, \ldots, \delta_{k}$ dos ângulos de $Q$, a soma acima continua sendo 0 e a órbita se mantém, logo $\Gamma$ é estável.

Teorema 2.0.2. Toda órbita periódica em um polígono cujos ângulos são racionalmente independentes (se existir) é estável.

Demonstração. Lembramos que um polígono ${ }^{1}$ de $k$ lados tem ângulos $\alpha_{1} \ldots \alpha_{k}$ racionalmente independentes se $n_{1} \alpha_{1}+\cdots+n_{k} \alpha_{k}=0$ com $n_{1}, \ldots n_{k} \in \mathbb{Z}$ implica que $n_{1}=\cdots=n_{k}=0$.

Seja $\Gamma$ uma órbita bilhar periódica num polígono de ângulos racionalmente independentes $Q$ e $\omega(\Gamma)$ a palavra de tamanho par definida por esta órbita.

Fixamos coordenadas cartesianas no plano e consideramos os ângulos $\alpha_{i}$ que cada lado $i$ de $Q$ forma com a horizontal.

Como $\Gamma$ é periódica, a soma

$$
\left(\alpha_{\omega_{1}}-\alpha_{\omega_{2}}\right)+\cdots+\left(\alpha_{\omega_{N-1}}-\alpha_{\omega_{N}}\right)
$$

\footnotetext{
${ }^{1}$ Em [VGS92], os autores chamam polígonos cujos ângulos são racionalmente independentes de polígonos típicos.
} 

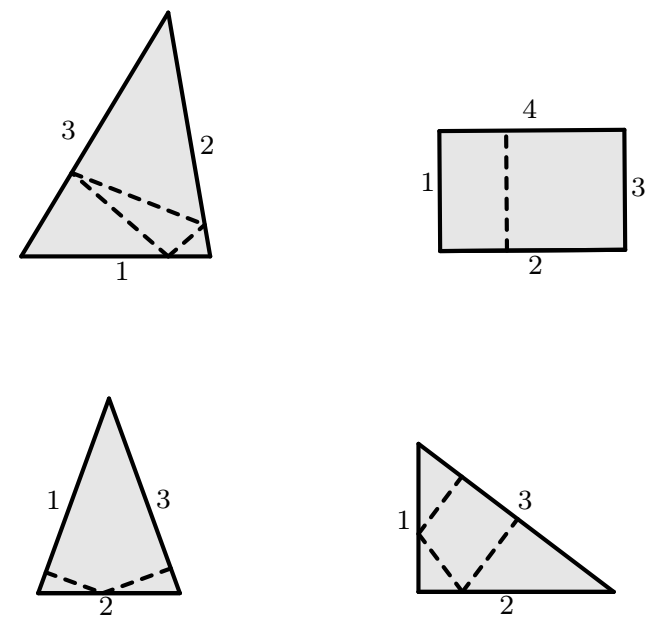

Figura 2.1: A órbita 123123 no triângulo agudo é estável; a órbita 24 no retângulo é instável; a órbita 1232 no triângulo isósceles é instável e, por fim a órbita 312321 no triângulo retângulo é instável.

é múltipla de $2 \pi$.

Supomos que a palavra $\omega(\Gamma)$ não é estável. Então temos

$$
m_{1} \alpha_{\omega_{1}}+m_{2} \alpha_{\omega_{2}}+\cdots+m_{K-1} \alpha_{\omega_{N-1}}+m_{K} \alpha_{\omega_{N}}=2 m \pi,
$$

com $m, m_{i} \in \mathbb{Z}$, e não temos $m=m_{1}=\cdots=m_{K}=0$. Logo existiria uma relação racional entre os ângulos de $Q$, o que não pode ocorrer.

Logo, $\Gamma$ é estável.

Agora, voltamos ao caso específico dos triângulos obtusos e ao espaço $\Delta$ de parâmetros que os representa.

Definição 2.0.4. Dada uma palavra $\omega$, o ladrilho de órbita de $\omega$, denotado por $O(\omega)$, é o conjunto de todos os pontos de $\Delta$ tais que $\omega$ codifica uma órbita bilhar periódica no triângulo representado por este ponto.

Se $\omega$ for estável, então $O(\omega)$ é um aberto. Se $\omega$ for instável, então para que o primeiro e o último lado do desdobramento sejam paralelos os ângulos devem satisfazer

$$
\left(\alpha_{\omega_{1}}-\alpha_{\omega_{2}}\right)+\cdots+\left(\alpha_{\omega_{N-1}}-\alpha_{\omega_{N}}\right)=2 m \pi,
$$

para algum $m$ inteiro, como vimos na demonstração do teorema 2.0.1. Mas, pela instabilidade, os termos desta soma não se cancelam em pares. Logo os ângulos $\alpha$ e $\beta$ do triângulo devem satisfazer $k \alpha+l \beta=n \pi$, com $k, l, n \in \mathbb{Z}$ e $O(\omega) \subset \Delta$ é formado por uma união finita de segmentos e tem interior vazio. É o caso da órbita 312321, que só existe em triângulos retângulos e das órbitas dos teoremas 1.1.1 e 1.1.2 (veja a figura 2.2). Por isso, vamos nos concentrar em ladrilhos de órbitas estáveis.

Note que, em vista da proposição 0.0.1, são necessários infinitos ladrilhos de órbitas estáveis para cobrir o espaço dos parâmetros, uma vez que alguns triângulos exigem órbitas periódicas arbitrariamente longas.

Vamos estudar algumas órbitas estáveis e calcular seus ladrilhos. Começaremos com um exemplo de triângulo agudo mais simples para ilustrar a ideia que será usada em famílias de triângulos obtusos no decorrer deste trabalho.

Apesar de vermos 3 exemplos de órbitas perpendiculares instáveis na figura 2.1, o triângulo agudo $\left(\frac{\pi}{3}, \frac{\pi}{4}\right)$ possui uma órbita periódica perpendicular e estável. Se codificarmos o lados $\overline{A B}, \overline{B C}$ e $\overline{A C}$ como 1, 2 e 3 respectivamente, esta órbita pode ser representada com a palavra 132132123123, que é, de fato, estável e define o feixe tracejado na figura 2.3. 

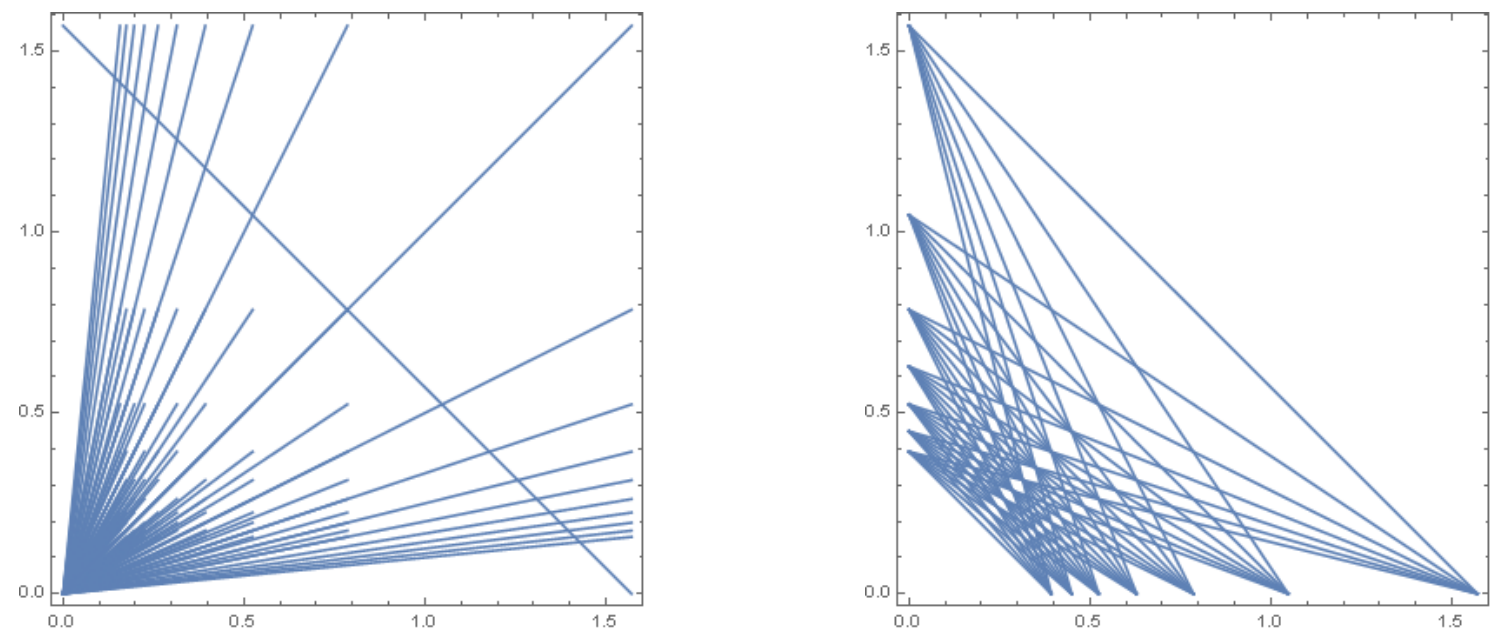

Figura 2.2: Á esquerda vemos os ladrilhos de órbita gerados pelo teorema 1.1 .1 e à esquerda os do teorema 1.1.2.

Agora calculemos a vizinhança de $\left(\frac{\pi}{3}, \frac{\pi}{4}\right)$ onde esta órbita se mantém. Como 132132123123 é estável, o último e o primeiro lado do desdobramento são paralelos. Basta identificar condições sobre $\alpha$ e $\beta$ tais que a faixa paralela ligando esses lados contenha uma faixa menor contida no desdobramento.

Fixamos o lado $\overline{A B}$ horizontalmente e o vértice $A$ na origem. Os lados $\overline{A B}, \overline{B C}$ e $\overline{A C}$ tem comprimento $c$, $a$ e $b$, respectivamente. Seja $\gamma=\pi-\alpha-\beta$ o ângulo $A \hat{C} B$.

Para que este desdobramento continue tendo uma órbita bilhar perpendicular, é necessário que os pontos $A, E$ e $O$ estejam à direita de $C, D$ e $F$. Vamos calcular a coordenada de $x$ de cada um desses pontos e depois ver as condições necessárias e suficientes para que $0<x_{C}, 0<x_{D}, 0<x_{F}$, $x_{E}<x_{C}, x_{E}<x_{D}, x_{E}<x_{F}, x_{O}<x_{C}, x_{O}<x_{D}$ e $x_{O}<x_{F}$.

$$
\begin{aligned}
& x_{C}=b \cos \alpha \\
& x_{E}=b \cos \alpha+a \cos (2 \alpha+\beta) \\
& x_{D}=b \cos \alpha+a \cos (2 \alpha+\beta)+c \cos (2 \alpha+2 \beta-\pi) \\
& x_{O}=b \cos \alpha+a \cos (2 \alpha+\beta)+c \cos (2 \alpha+2 \beta-\pi)+b \cos (\alpha+2 \beta) \\
& x_{F}=b \cos \alpha+a \cos (2 \alpha+\beta)+c \cos (2 \alpha+2 \beta-\pi)+b \cos (\alpha+2 \beta)+a \cos \beta
\end{aligned}
$$

Comecemos com os itens mais fáceis de calcular, que são os consecutivos da lista de igualdades acima:

$$
\begin{aligned}
0<x_{C} & \Leftrightarrow 0<b \cos \alpha \Leftrightarrow \alpha<\frac{\pi}{2} ; \\
x_{E}<x_{C} & \Leftrightarrow a \cos (2 \alpha+\beta)<0 \Leftrightarrow \frac{\pi}{2}<2 \alpha+\beta<\frac{3 \pi}{2} ;
\end{aligned}
$$

$$
\begin{aligned}
& x_{E}<x_{D} \Leftrightarrow 0<c \cos (2 \alpha+2 \beta-\pi) \Leftrightarrow \frac{-\pi}{2}<2 \alpha+2 \beta-\pi<\frac{\pi}{2} \Leftrightarrow \frac{\pi}{2}<\alpha+\beta<\frac{3 \pi}{4} ; \\
& x_{O}<x_{D} \Leftrightarrow b \cos (\alpha+2 \beta)<0 \Leftrightarrow \frac{\pi}{2}<\alpha+2 \beta<\frac{3 \pi}{2} ; \\
& x_{O}<x_{F} \Leftrightarrow 0<a \cos \beta \Leftrightarrow \beta<\frac{\pi}{2} .
\end{aligned}
$$

Para os demais, como os comprimentos dos lados do triângulo não importam, apenas os ângulos, 


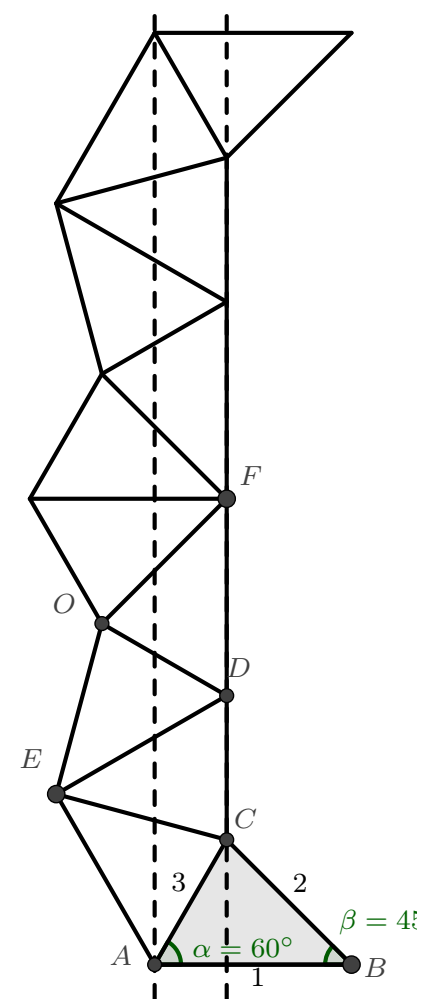

Figura 2.3: Desdobramento do triângulo $\left(\frac{\pi}{3}, \frac{\pi}{4}\right)$ com relação a uma órbita perpendicular

vamos usar a lei dos senos com triângulo inscrito numa circunferência de diâmetro 1:

$$
\frac{a}{\sin \alpha}=\frac{b}{\sin \beta}=\frac{c}{\sin \gamma}=1
$$

Então podemos substituir $a=\sin \alpha, b=\sin \beta$ e $c=\sin (\alpha+\beta)$. Isso junto com algumas identidades trigonométricas nos dão:

$$
\begin{aligned}
0<x_{D} & \Leftrightarrow 0<\sin \beta \cos \alpha+\sin \alpha \cos (2 \alpha+\beta)-\sin (\alpha+\beta) \cos (2 \alpha+2 \beta)=2 \sin \beta \sin (\alpha+\beta) \sin (2 \alpha+\beta) \\
& \Leftrightarrow 0<2 \alpha+\beta<\pi ; \\
x_{E}<x_{F} & \Leftrightarrow 0<-\sin (\alpha+\beta) \cos (2 \alpha+2 \beta)+\sin \beta \cos (\alpha+2 \beta)+\sin \alpha \cos \beta=2 \sin \alpha \sin (\alpha+\beta) \sin (\alpha+2 \beta) \\
& \Leftrightarrow 0<\alpha+2 \beta<\pi ; \\
x_{O}<x_{C} & \Leftrightarrow 0>\sin \alpha \cos (2 \alpha+\beta)-\sin (\alpha+\beta) \cos (2 \alpha+2 \beta)+\sin \beta \cos (\alpha+2 \beta)=2 \sin \alpha \sin \beta \sin (2 \alpha+2 \beta) \\
& \Leftrightarrow \frac{\pi}{2}<\alpha+\beta<\pi ; \\
0<x_{F} & \Leftrightarrow 0<\sin \beta \cos \alpha+\sin \alpha \cos (2 \alpha+\beta)-\sin (\alpha+\beta) \cos (2 \alpha+2 \beta)+\sin \beta \cos (\alpha+2 \beta)+\sin \alpha \cos \beta \\
& =-\sin (\alpha+\beta)(\cos (2 \alpha+2 \beta)+\cos (2 \alpha)+\sin (2 \beta)) \Leftrightarrow 0<\cos (2 \alpha+2 \beta)+\cos (2 \alpha)+\sin (2 \beta \\
& \Leftrightarrow 2 \cos (\alpha+\beta) \cos (\alpha-\beta)>2 \cos ^{2}(\alpha+\beta)-1 .
\end{aligned}
$$

Estas desigualdades nos dão que a órbita só existe em triângulos agudos, $2 \alpha+\beta, \alpha+2 \beta \in\left(\frac{\pi}{2}, \pi\right)$ e que $\alpha$ e $\beta$ satisfazem $2 \cos (\alpha+\beta) \cos (\alpha-\beta)>2 \cos ^{2}(\alpha+\beta)-1$. Esta região está ilustrada na figura 2.4 . 


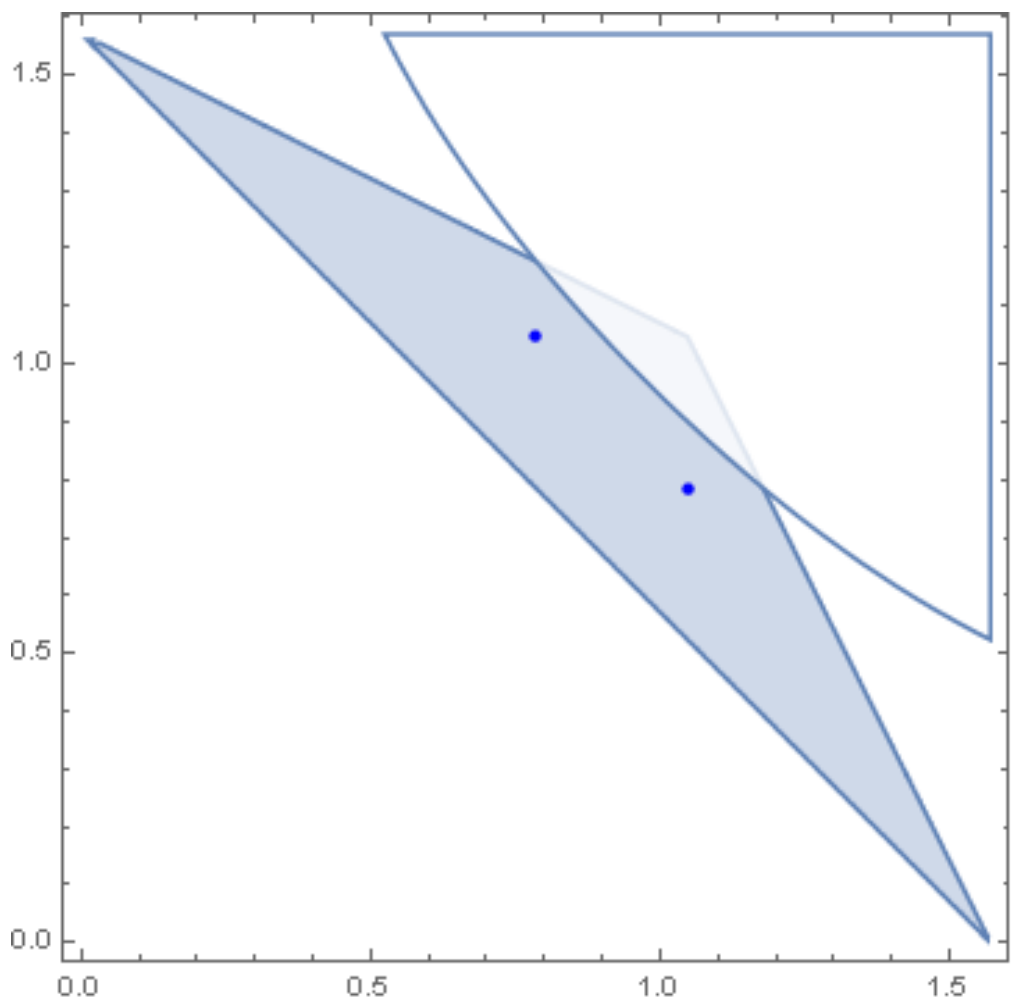

Figura 2.4: $O(132132123123)$. Os pontos destacados são $\left(\frac{\pi}{3}, \frac{\pi}{4}\right)$ e $\left(\frac{\pi}{4}, \frac{\pi}{3}\right)$.

\section{1 Órbitas ímpares}

Toda órbita bilhar periódica com um número ímpar de segmentos é estável pelo teorema 2.0.1, logo é interessante estudar órbitas ímpares e seus ladrilhos de órbita. Em [VGS92] temos uma condição suficiente para gerar uma família de órbitas ímpares.

Teorema 2.1.1. Seja $\triangle A B C$ obtuso com $\alpha$ e $\beta$ satisfazendo $k \alpha<\frac{\pi}{2}, l \beta<\frac{\pi}{2}$ e a desigualdade

$$
(1-\operatorname{cotg}(k \alpha) \tan (\alpha))(1-\operatorname{cotg}(l \beta) \tan (\beta))>1-\tan \alpha \tan \beta,
$$

com $k, l \in \mathbb{N}$. Então $\triangle A B C$ possui uma órbita periódica ímpar de $2(k+l)-1$ segmentos.

Demonstração. Seja $\triangle A N C$ o triângulo que tem como base o lado $A C$ e ângulo $\angle A=k \alpha$ e $\angle C=l \beta$. Este triângulo é agudo por hipótese. Neste triângulo, consideramos os pontos $H_{a}, H_{c} \mathrm{e}$ $H_{n}$ base das alturas.

O triângulo $\triangle H_{a} H_{c} H_{n}$ é a órbita periódica de $2(k+l)-1$ segmentos de $\triangle A B C$ se o seguimento $H_{c} H_{a}$ está abaixo do ponto $B$, como na figura 2.5. Vamos calcular os pontos e retas associados a este triângulo e mostrar que esta condição é satisfeita se e somente se a desigualdade 2.1 é satisfeita. Para isso, vamos fixar $A=(0,0)$ e deixar que o comprimento do lado $A C$ seja 1.

Temos: $A=(0,0), B=\left(\frac{\tan (\beta)}{\tan (\alpha)+\tan (\beta)}, \frac{\tan (\alpha) \tan (\beta)}{\tan (\alpha)+\tan (\beta)}\right)$ e $C=(1,0)$. Queremos as coordenadas de $\stackrel{H_{G}}{\stackrel{A}{A}}$ e $H_{a}$.

A reta $\overleftrightarrow{A N}$ tem equação $y=\tan (k \alpha) x$ e a reta $\overleftrightarrow{A N} \perp$ perpendicular a $\overleftrightarrow{A N}$ passando por $C$ é $y=\operatorname{cotg}(k \alpha)(1-x)$. Logo, como $H_{c}$ é o ponto de interseção de $\overleftrightarrow{A N}$ e $\overleftrightarrow{A N} \perp$

$$
H_{c}=\left(x_{c}, y_{c}\right)=\left(\frac{1}{\tan ^{2}(k \alpha)+1}, \frac{\tan (k \alpha)}{\tan ^{2}(k \alpha)+1}\right)=\left(\frac{\operatorname{cotg}^{2}(k \alpha)}{\operatorname{cotg}^{2}(k \alpha)+1}, \frac{\operatorname{cotg}(k \alpha)}{\operatorname{cotg}^{2}(k \alpha)+1}\right) .
$$




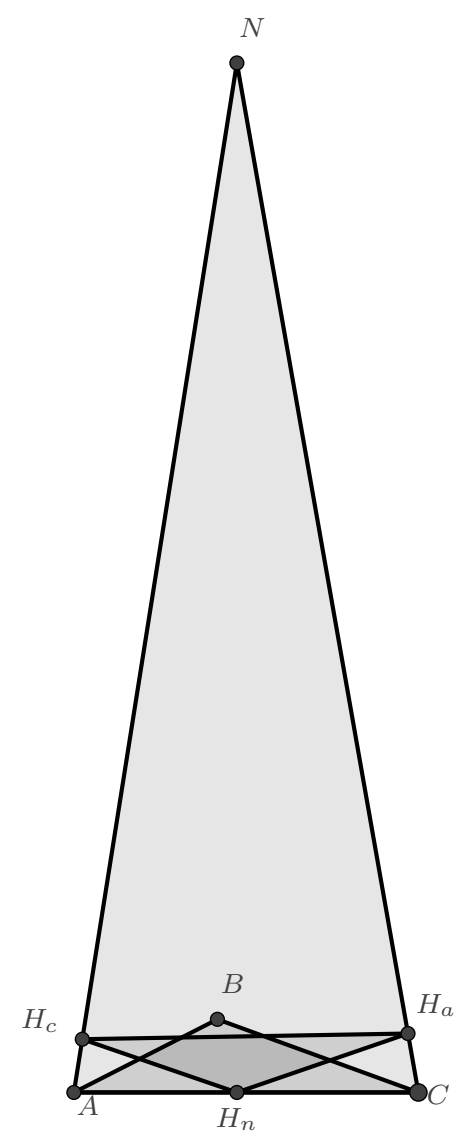

Figura 2.5: $\triangle A N C$

Do mesmo modo, calculamos a reta $\overleftrightarrow{C N}$, cuja equação é $y=\tan (l \beta)(1-x)$. Sua perpendicular passando por $A$ é a reta $\overleftrightarrow{C N^{\perp}}$, de equação $y=\operatorname{cotg}(l \beta) x$. Assim,

$$
H_{a}=\left(x_{a}, y_{a}\right)=\left(\frac{1}{\operatorname{cotg}^{2}(l \beta)+1}, \frac{\operatorname{cotg}(l \beta)}{\operatorname{cotg}^{2}(l \beta)+1}\right) .
$$

A equação da reta $\overleftrightarrow{H_{C} H_{A}}$ é $y=\frac{y_{a}-y_{c}}{x_{a}-x_{c}} x+\frac{x_{a} y_{c}-x_{c} y_{a}}{x_{a}-x_{c}}$. Então queremos mostrar que a desigualdade 2.1 é satisfeita se, e somente se,

$$
\frac{\tan (\alpha) \tan (\beta)}{\tan (\alpha)+\tan (\beta)}>\frac{y_{a}-y_{c}}{x_{a}-x_{c}} \frac{\tan (\beta)}{\tan (\alpha)+\tan (\beta)}+\frac{x_{a} y_{c}-x_{c} y_{a}}{x_{a}-x_{c}} .
$$

Como $x_{a}-x_{c}>0$, isto é equivalente a

$$
\left(x_{a}-x_{c}\right) \frac{\tan (\alpha) \tan (\beta)}{\tan (\alpha)+\tan (\beta)}>\left(y_{a}-y_{c}\right) \frac{\tan (\beta)}{\tan (\alpha)+\tan (\beta)}+\left(x_{a} y_{c}-x_{c} y_{a}\right) .
$$

Seja $z=\left(\operatorname{cotg}^{2}(k \alpha)+1\right)\left(\operatorname{cotg}^{2}(l \beta)+1\right)$. Temos que

$$
\begin{gathered}
x_{a}-x_{c}=\frac{1-\operatorname{cotg}^{2}(k \alpha) \operatorname{cotg}^{2}(l \beta)}{z}, \\
y_{a}-y_{c}=\frac{(\operatorname{cotg}(l \beta)-\operatorname{cotg}(k \alpha))(1-\operatorname{cotg}(k \alpha) \operatorname{cotg}(l \beta))}{z} \\
\text { e } x_{a} y_{c}-x_{c} y_{a}=\frac{\operatorname{cotg}(k \alpha)(1-\operatorname{cotg}(k \alpha) \operatorname{cotg}(l \beta))}{z} .
\end{gathered}
$$


Logo, como $z$ é positivo, a equação 2.2 é equivalente a

$$
\begin{aligned}
& \left(1-\operatorname{cotg}^{2}(k \alpha) \operatorname{cotg}^{2}(l \beta)\right) \frac{\tan (\alpha) \tan (\beta)}{\tan (\alpha)+\tan (\beta)}> \\
& (\operatorname{cotg}(l \beta)-\operatorname{cotg}(k \alpha))(1-\operatorname{cotg}(k \alpha) \operatorname{cotg}(l \beta)) \frac{\tan (\beta)}{\tan (\alpha)+\tan (\beta)}+\operatorname{cotg}(k \alpha)(1-\operatorname{cotg}(k \alpha) \operatorname{cotg}(l \beta)),
\end{aligned}
$$

que, por sua vez, é equivalente a

$\left(1-\operatorname{cotg}^{2}(k \alpha) \operatorname{cotg}^{2}(l \beta)\right)(\tan (\alpha) \tan (\beta))>(1-\operatorname{cotg}(k \alpha) \operatorname{cotg}(l \beta))(\tan (\alpha) \operatorname{cotg}(k \alpha)+\tan (\beta) \operatorname{cotg}(l \beta))$.

Como $\left(1-\operatorname{cotg}^{2}(k \alpha) \operatorname{cotg}^{2}(l \beta)\right)=(1+\operatorname{cotg}(k \alpha) \operatorname{cotg}(l \beta))(1-\operatorname{cotg}(k \alpha) \operatorname{cotg}(l \beta))$, então a desigualdade acima é satisfeita se e somente se

$$
\begin{aligned}
& \tan (\alpha) \tan (\beta)>\tan (\alpha) \operatorname{cotg}(k \alpha)+\tan (\beta) \operatorname{cotg}(l \beta)-\tan (\alpha) \tan (\beta) \operatorname{cotg}(k \alpha) \operatorname{cotg}(l \beta) \\
& \Longleftrightarrow 1+\tan (\alpha) \tan (\beta) \operatorname{cotg}(k \alpha) \operatorname{cotg}(l \beta)-\tan (\alpha) \operatorname{cotg}(k \alpha)-\tan (\beta) \operatorname{cotg}(l \beta)>1-\tan (\alpha) \tan (\beta)
\end{aligned}
$$

que é equivalente a 2.1.

Os pontos $(\alpha, \beta)$ que satisfazem a desigualdade 2.1 definem uma família bi-infinita de abertos no espaço dos parâmetros onde existe a órbita ímpar do teorema 2.1.1.

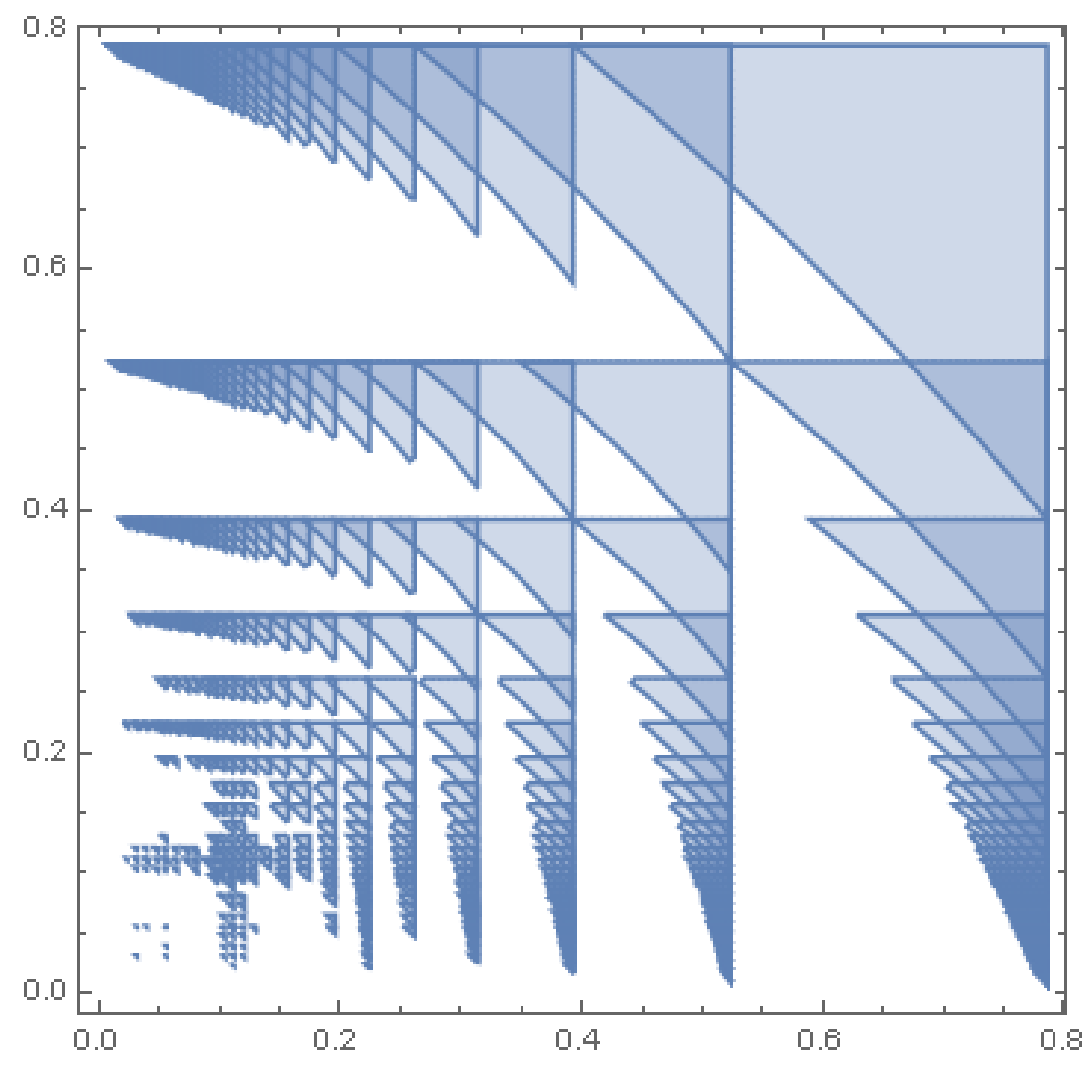

Figura 2.6: Subconjunto do espaço do parâmetros dos pontos que satisfazem as hipóteses do teorema 2.1.1. 


\subsection{Famílias de órbitas ímpares}

Em [HH00], inspirados pelo caso do teorema 2.1.1, os autores encontraram outras famílias de órbitas ímpares.

Definição 2.2.1. Uma família infinita de palavras num alfabeto de três símbolos é dita geradora se para toda palavra desta família existe um triângulo onde ela codifica uma órbita periódica estável. As palavras destas famílias são ditas geradores.

Como toda órbita ímpar é estável, a ideia é encontrar famílias geradoras nas quais todas as palavras são ímpares. Para encontrar geradores, Halbeisen e Hungerbühler partiram de casos degenerados e calcularam as perturbações e condições necessárias para que houvesse uma órbita periódica nestes casos.

Estes casos degenerados surgem de dois tipos de configuração. O primeiro tipo, denotado por $\left\langle n_{k}, \ldots, n_{1}|\mathcal{B}| N_{1}, \ldots, N_{l}\right\rangle$, descreve a seguinte configuração do desdobramento: o lado $\overline{A B}$ é fixado horizontalmente e a partir do lado 1 (à direita), são realizadas as reflexões codificadas $^{2}$ por $(13)^{N_{1} / 2}(23)^{N_{2} / 2} \ldots(13)^{N_{l-1} / 2}(23)^{N_{l} / 2}$ se $l$ for par e $(13)^{N_{1} / 2}(23)^{N_{2} / 2} \ldots(13)^{N_{l} / 2}$ se $l$ for ímpar. A partir do lado 2 , à esquerda, são realizadas reflexões codificadas por $(23)^{n_{1} / 2}(13)^{n_{2} / 2} \ldots(23)^{n_{k-1} / 2}(13)^{n_{k} / 2}$ se $k$ for par e $(23)^{n_{1} / 2}(13)^{n_{2} / 2} \ldots(23)^{n_{k} / 2}$ se $k$ for ímpar. Veja a figura 2.7 .

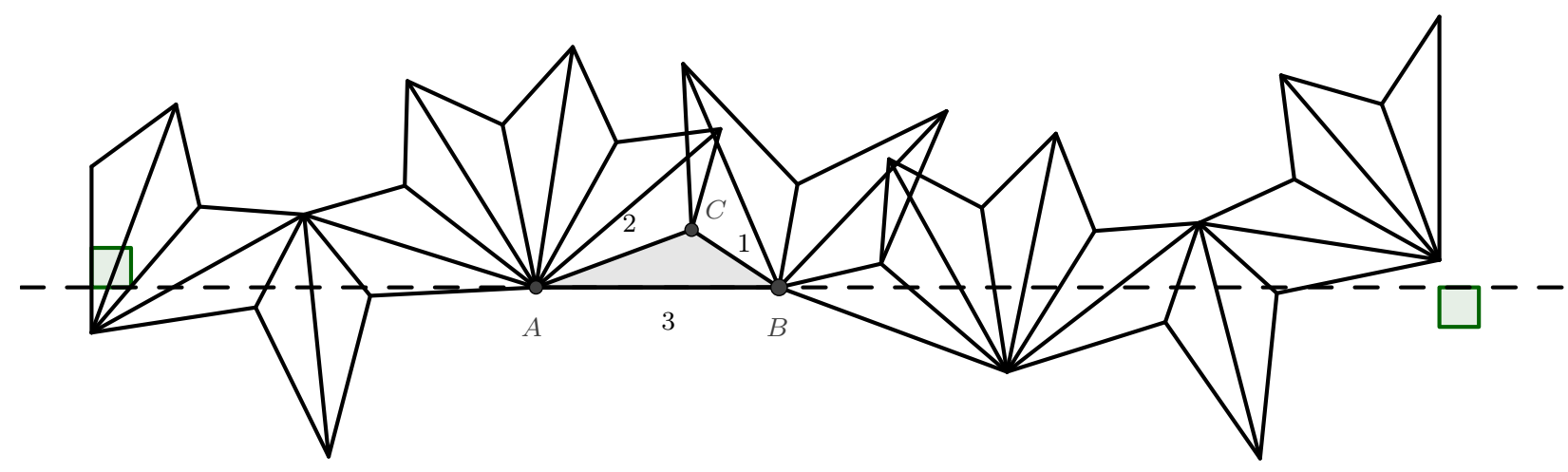

Figura 2.7: Configuração $\langle 2,4,8|\mathcal{B}| 5,6,4,3\rangle$. A órbita degenerada que percorre o lado 3 .

O segundo tipo é $\left\langle n_{k}, \ldots, n_{1}|\mathcal{L}| N_{1}, \ldots, N_{l}\right\rangle$ e descreve a seguinte configuração do desdobramento: o lado $\overline{A B}$ é fixado horizontalmente e a partir do lado 1 (à direita), são realizadas as reflexões codificadas por $(13)^{N_{1} / 2}(23)^{N_{2} / 2} \ldots(13)^{N_{l-1} / 2}(23)^{N_{l} / 2}$ se $l$ for par e $(13)^{N_{1} / 2}(23)^{N_{2} / 2} \ldots(13)^{N_{l} / 2}$ se $l$ for ímpar. A partir do lado 3 (para baixo) são realizadas reflexões codificadas por $(32)^{n_{1} / 2}(13)^{n_{2} / 2} \ldots(32)^{n_{k-1} / 2}(13)^{n_{k} / 2}$ se $k$ for par e $(32)^{n_{1} / 2}(13)^{n_{2} / 2} \ldots(32)^{n_{k} / 2}$ se $k$ for ímpar. Veja a figura 2.8 .

Em ambos os casos, calculamos os valores de $\alpha$ e $\beta$ tais que o segmento tracejado faz ângulos retos com o primeiro e o último lado do desdobramento. Feito isso, perturbamos estes valores para criar ângulos agudos e assim criar uma configuração não degenerada onde existe a órbita periódica. Veja a figura 2.9 .

Teorema 2.2.1. Para cada configuração a seguir, conseguimos uma família geradora de palavras:

(a) A configuração $\left\langle n_{1}|\mathcal{B}| 2 N_{1}, N_{2}\right\rangle$ nos dá a família

$$
\left\{312(32)^{k-1}(31)^{m}(32)^{n}(31)^{m-1}: n<k \leq n \frac{2 m+1}{2 m-1}, k \geq \frac{2 m+n}{2 m-1}\right\} ;
$$

\footnotetext{
${ }^{2}$ Se $N_{i}$ for ímpar $(A B)^{N_{i} / 2}=(A B)^{\left(N_{i}-1\right) / 2} A$. A notação $(A B)^{k}$ representa a repetição da palavra $A B k$ vezes, e usamos por padrão um expoente natural. Neste caso, cometemos o abuso de linguagem de "elevar a meio" uma palavra de tamanho dois para não carregar a notação.
} 


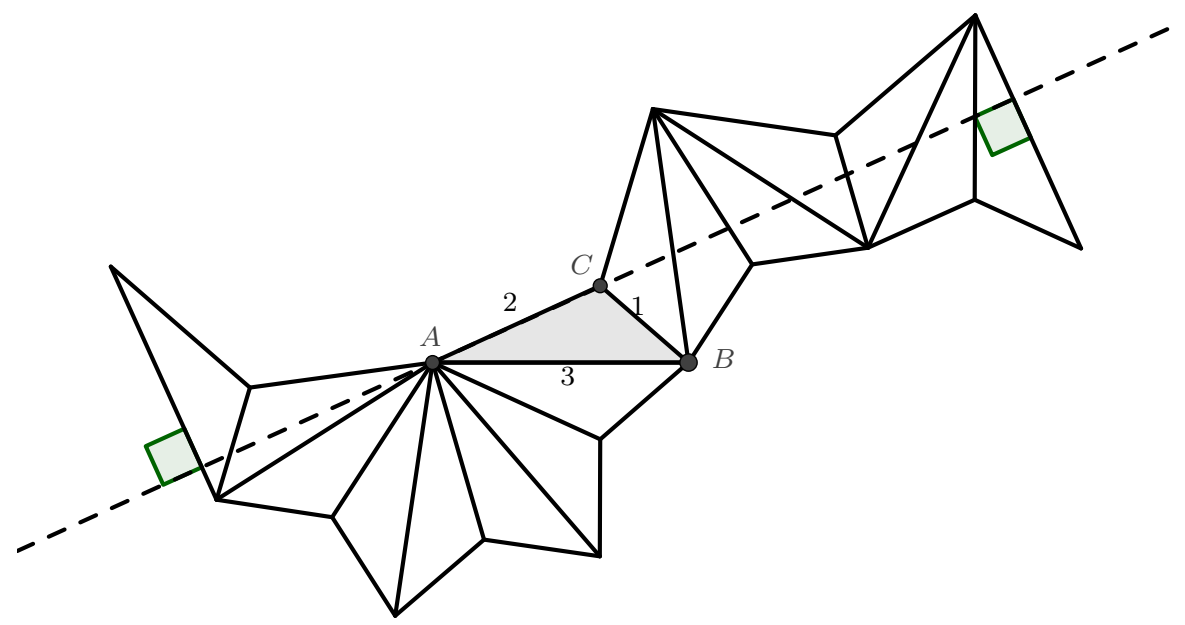

Figura 2.8: Configuração $\langle 1,7|\mathcal{L}| 2,2,2,1\rangle$. A órbita degenerada que percorre o lado 2 .

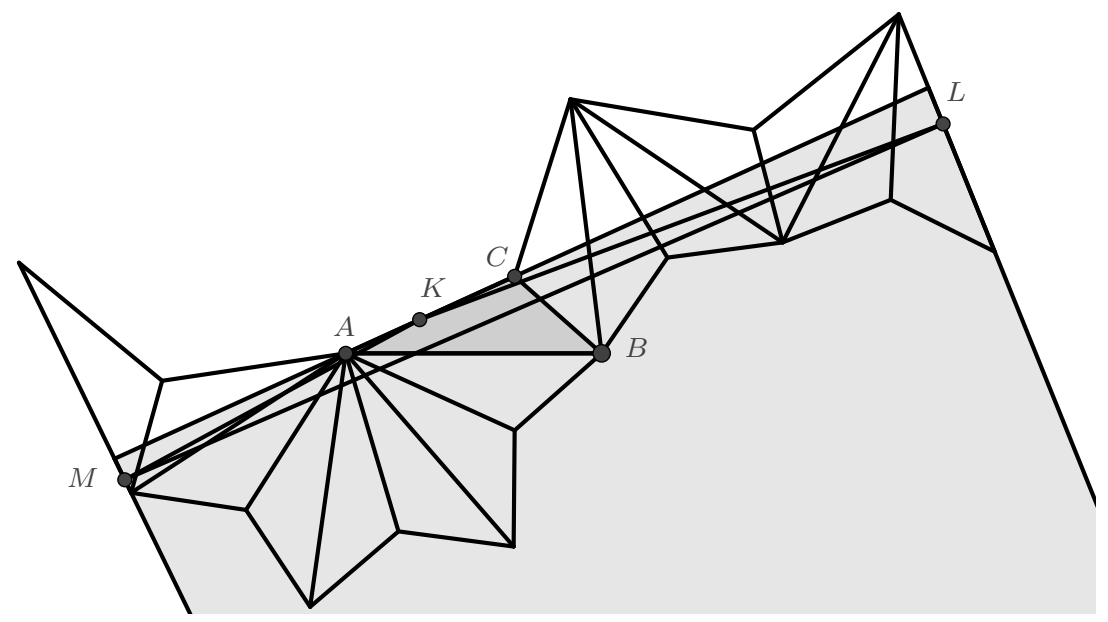

Figura 2.9: Uma pequena deformação de $\alpha$ e $\beta$ no triângulo da figura 2.8 nos da uma órbita periódica. A região escura é uma parte do triângulo agudo onde $K, L$ e $M$ são os pés das alturas.

(b) A configuração $\left\langle n_{1}|\mathcal{B}| 2 N_{1}+1, N_{2}\right\rangle$ nos dá a família

$$
\left\{312(32)^{k-1}(31)^{m} 2(32)^{n-1} 1(31)^{m-2}: 2 \leq n \leq k, 2 \leq m<\frac{3 k-n}{2(k-n)}\right\}
$$

(c) A configuração $\left\langle n_{2}, 2 n_{1}|\mathcal{B}| 2 N_{1}, N_{2}\right\rangle$ nos dá a família

$$
\begin{aligned}
& \left\{312(32)^{l-1}(31)^{k}(32)^{l}(31)^{m}(32)^{n}(31)^{m-1}: n<2 l,\right. \\
& \left.\quad \max \left(\frac{k+n+2 l+k n}{4 l-2}, \frac{k(l+n)}{2 l}, \frac{n k}{2 l-n}\right)<m<\min \left(\frac{k\left(l+n+\frac{1}{2}\right)}{2 l-1}, \frac{n\left(k+\frac{1}{2}\right)+l}{2 l-n}\right)\right\} ;
\end{aligned}
$$

(d) A configuração $\left\langle n_{2}, 2 n_{1}|\mathcal{B}| 2 N_{1}+1, N_{2}\right\rangle$ nos dá a família

$$
\begin{aligned}
& \left\{312(32)^{l-1}(31)^{k}(32)^{l}(31)^{m} 2(32)^{n-1} 1(31)^{m-2}: n<2 l,\right. \\
& \left.\quad \max \left(\frac{3 k+6 l-n-k n}{4 l-2}, \frac{k(3 l-n)}{2 l}, \frac{n k}{2 l-n}\right)<m<\min \left(\frac{k\left(3 l-n+\frac{3}{2}\right)}{2 l-1}, \frac{n\left(k-\frac{1}{2}\right)+3 l}{2 l-n}\right)\right\} ;
\end{aligned}
$$


(e) A configuração $\left\langle n_{2}, 2 n_{1}+1|\mathcal{B}| 2 N_{1}+1, N_{2}\right\rangle$ nos dá a família

$$
\begin{aligned}
& \left\{312(32)^{l-1} 1(31)^{k-1} 2(32)^{l-1}(31)^{m} 2(32)^{n-1} 1(31)^{m-2}:\right. \\
& \left.\quad 3\left(l-\frac{1}{2}\right)<\frac{4 l m-k n}{2 m-k}<3 l, 3\left(m-\frac{1}{2}\right)<\frac{4 l m-k n}{2 n-l}<3 m\right\} ;
\end{aligned}
$$

(f) A configuração $\left\langle n_{1}|\mathcal{L}| 2 N_{1}, N_{2}\right\rangle$ nos dá a família

$$
\begin{aligned}
& \left\{312(32)^{k}(31)^{m}(32)^{n+1}(31)^{m-1}:\right. \\
& 2+n<k(2 k-n), 2 k m(2 k+2)<(k+1)(m+1)(2 k+2+n)\} ;
\end{aligned}
$$

(g) A configuração $\left\langle n_{2}, 2 n_{1}+1|\mathcal{L}| N_{1}\right\rangle$ nos dá a família

$$
\left\{312(32)^{k}(31)^{l}(32)^{k}(31)^{m-1}: \max (2 m, 2 k(l-m))<l+m\right\} ;
$$

(h) A configuração $\left\langle n_{2}, 2 n_{1}|\mathcal{L}| N_{1}\right\rangle$ nos dá a família

$$
\left\{312(32)^{k} 1(31)^{l-1} 2(32)^{k-1}(31)^{m-1}: m<l<3 l, 2 k(l-m)<l+m\right\}
$$

(i) A configuração $\left\langle n_{2}, 2 n_{1}+1|\mathcal{L}| 2 N_{1}, N_{2}\right\rangle$ nos dá a família

$$
\begin{array}{r}
\left\{312(32)^{k}(31)^{l}(32)^{k}(31)^{m}(32)^{n+1}(31)^{m-1}:(m+l)(n+1)<2 k m<l(k+1+n),\right. \\
\left.2 k<\frac{(n+1)(l+m+1)}{m-1}, \max (l(2 k+1+2 n), l n)<2 m(2 k+1)\right\}
\end{array}
$$

(j) A configuração $\left\langle n_{2}, 2 n_{1}|\mathcal{L}| 2 N_{1} N_{2}\right\rangle$ nos dá a família

$$
\left\{\begin{array}{l}
\left\{312(32)^{k} 1(31)^{l-1} 2(32)^{k-1}(31)^{m}(32)^{n+1}(31)^{m-1}: l<6 m, 2 m(k-1)<l(k+n),\right. \\
\quad l(2 k+1+2 n)<2 m(2 k+1), m(3 n+5-2 k)<l(n+1)<m(3 n+5-2 k)+2 k+1+3 n\} ;
\end{array}\right.
$$

Demonstração. Demonstremos o caso (a). Definimos $k:=n_{1}+1 \geq 1, n:=N_{2}+1 \geq 1$ e $m:=N_{1} \geq 1$. Denotamos ângulos que o primeiro e o último lado do desdobramento formam com a horizontal por $\varepsilon_{1}$ e $\varepsilon_{2}$, respectivamente. Veja a figura 2.10 .

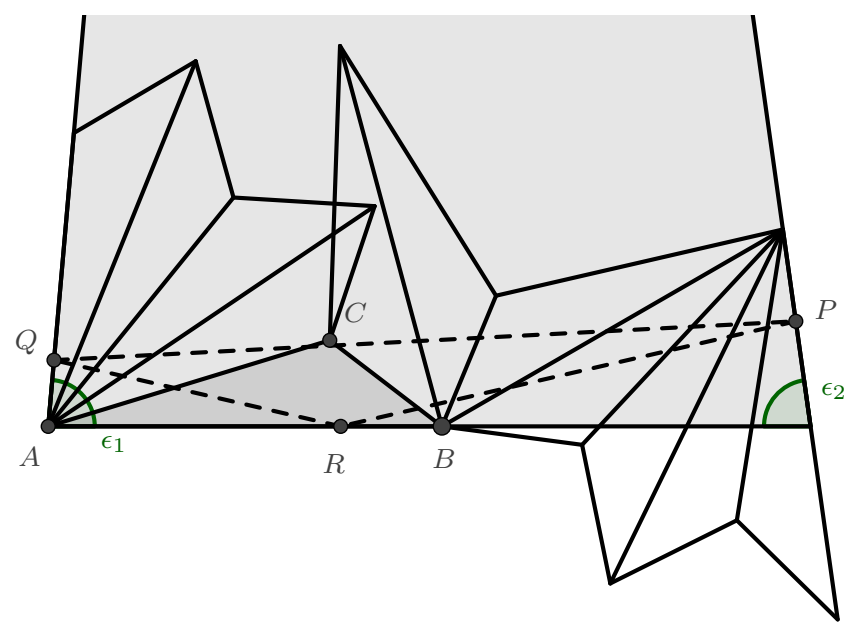

Figura 2.10: $\langle 4|\mathcal{B}| 4,3\rangle$, o triângulo agudo de ângulos $\varepsilon_{1}$ e $\varepsilon_{2}$ com a órbita periódica $P Q R$. 
Note que

$$
\left\{\begin{array}{l}
\varepsilon_{1}=k \alpha \\
\varepsilon_{2}=2 m \beta-n \alpha .
\end{array}\right.
$$

Tomando $\alpha_{0}=\frac{\pi}{2 k}$ e $\beta_{0}=\frac{\pi(k+n)}{4 k m}$, temos $\varepsilon_{1}=\varepsilon_{2}=\frac{\pi}{2}$. Ainda, a transformação $(\alpha, \beta) \mapsto$ $\left(\varepsilon_{1}, \varepsilon_{2}\right)$ tem Jacobiana

$$
\left|\begin{array}{cc}
k & 0 \\
-n & 2 m
\end{array}\right|=2 k m>0
$$

logo, pelo teorema da função inversa, valores de $\varepsilon_{1}$ e $\varepsilon_{2}$ suficientemente próximos de $\frac{\pi}{2}$ são atingidos com pequenas perturbações de $\alpha_{0}$ e $\beta_{0}$.

Vejamos que se as condições $n<k \leq n \frac{2 m+1}{2 m-1}, k \geq \frac{2 m+n}{2 m-1}$ são satisfeitas, então existem $\alpha$ e $\beta$ tais que os segmentos $\overline{P Q}, \overline{Q R}$ e $\overline{R P}$ representam uma órbita periódica no triângulo de ângulos $\alpha$ e $\beta$.

Note que

(i) $n<k \Longrightarrow 2 m \beta_{0}<\pi$;

(ii) $k \leq n \frac{2 m+1}{2 m-1} \Longrightarrow(2 m+1) \beta_{0} \geq \pi$;

(iii) $k \geq \frac{2 m+n}{2 m-1} \Longrightarrow \alpha_{0}+\beta_{0} \leq \frac{\pi}{2}$.

Se temos $k<n \frac{2 m+1}{2 m-1}$ e $k>\frac{2 m+n}{2 m-1}$, então $(2 m+1) \beta_{0}>\pi$ e $\alpha_{0}+\beta_{0}<\frac{\pi}{2}$, o que implica que podemos escolher $(\alpha, \beta)$ tais que $\varepsilon_{1}$ e $\varepsilon_{2}$ são menores que $\frac{\pi}{2}, \alpha+\beta<\frac{\pi}{2}$ e os segmentos do triângulo $P Q R$ estão contidos no desdobramento de forma que $R$ está entre $A$ e $B$.

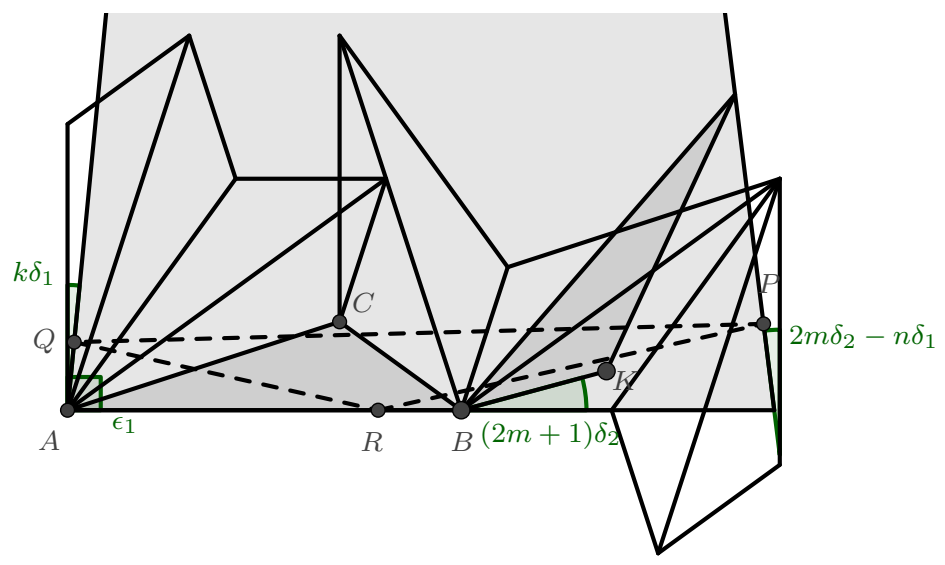

Figura 2.11: $\langle 4|\mathcal{B}| 4,2\rangle$, com $\alpha_{0}$ e $\beta_{0}$ temos $(2 m+1) \beta_{0}=\pi$. Com $\alpha=\alpha_{0}-\delta_{1}$ e $\beta=\beta_{0}-\delta_{2}$, os segmentos do triângulo $P Q R$ formam uma órbita periódica no desdobramento.

Se temos $k=n \frac{2 m+1}{2 m-1}$ em (ii), estamos no caso da figura 2.11. O desdobramento ilustra o caso $(2 m+1) \beta_{0}=\pi \operatorname{com} \varepsilon_{1}=\varepsilon_{2}=\frac{\pi}{2}$ atingidos ao tomar os valores $\alpha_{0}$ e $\beta_{0}$. Podemos tomar $\alpha$ e $\beta$ ligeiramente menores, $\alpha=\alpha_{0}-\delta_{1}$ e $\beta=\beta_{0}-\delta_{2}$, tais que $\alpha+\beta<\frac{\pi}{2}, \varepsilon_{1}<\frac{\pi}{2}, \varepsilon_{2}<\frac{\pi}{2}$ e o triângulo com ângulos $\varepsilon_{1}$ e $\varepsilon_{2}$, por ser agudo, possui a órbita definida pelas bases das alturas, tracejada na figura 2.11. De fato, basta tomar $\left(m-\frac{1}{2}\right) \delta_{2}>n \delta_{1}$, que nos dá

$$
m \delta_{2}-n \delta_{1}>\frac{1}{2} \delta_{2} \Longrightarrow 2 m \delta_{2}-n \delta_{1}>m \delta_{2}+\frac{1}{2} \delta_{2}=\frac{1}{2}(2 m-1) \delta_{2} .
$$


Isso implica que o segmento $\overline{P R}$ está acima de $K$, logo $P Q R$ é uma órbita periódica no triângulo de ângulos $\alpha$ e $\beta$.

Se $k=\frac{2 m+n}{2 m-1}$, então $\alpha_{0}+\beta_{0}=\frac{\pi}{2}$. Veja a figura 2.12 para o desdobramento da configuração $\langle 5|\mathcal{B}| 2,3\rangle$. Se o triângulo $A B C$ tiver ângulos $\alpha_{0}$ e $\beta_{0}$ ele será retângulo e $\varepsilon_{1}=\varepsilon_{2}=\frac{\pi}{2}$. Como no caso anterior, podemos tomar $\alpha$ e $\beta$ ligeiramente menores, $\alpha=\alpha_{0}-\delta_{1}$ e $\beta=\beta_{0}-\delta_{2}$, tais que $\alpha+\beta<\frac{\pi}{2}, \varepsilon_{1}<\frac{\pi}{2}, \varepsilon_{2}<\frac{\pi}{2}$ e o triângulo com ângulos $\varepsilon_{1}$ e $\varepsilon_{2}$, por ser agudo, possui a órbita definida pelas bases das alturas, tracejada na figura 2.12. É possível escolher $\alpha$ e $\beta$ tal que $R$ está entre $A$ e $B$ e $\overline{P Q}$ está abaixo de $\mathrm{C}$.

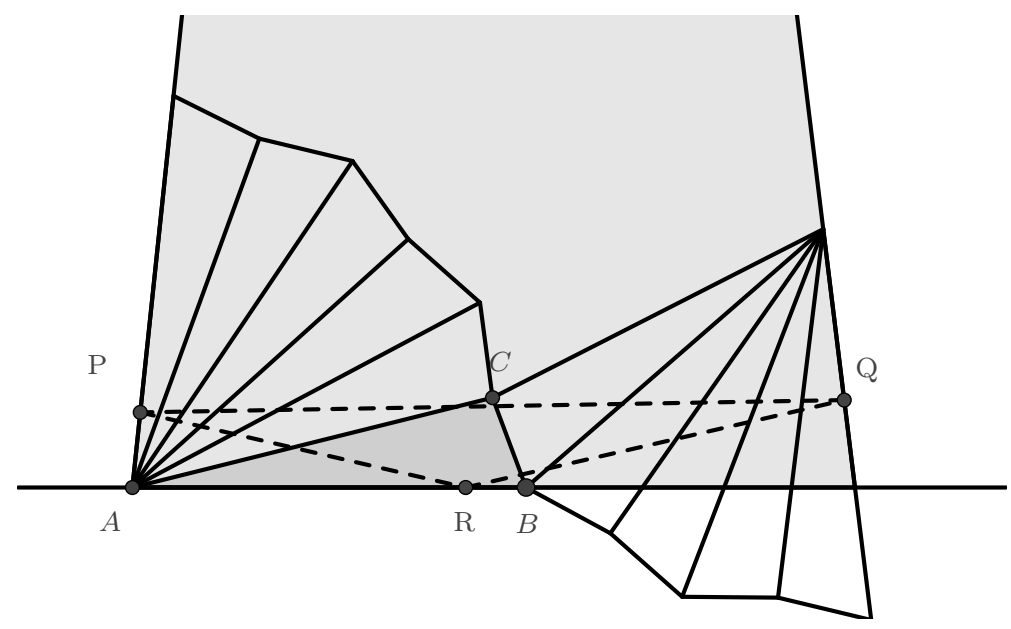

Figura 2.12: $\langle 5|\mathcal{B}| 2,3\rangle$. Os segmentos do triângulo $P Q R$ formam uma órbita periódica no desdobramento.

Logo, as condições (i), (ii) e (iii) implicam que existem $\alpha$ e $\beta$ tais que a configuração $\left\langle n_{1}|\mathcal{B}| 2 N_{1}, N_{2}\right\rangle$ gera uma órbita periódica. Isso nos dá a família do caso (a).

Vejamos agora o caso (f). Definimos $k:=n_{1}+1 \geq 1, m:=N_{1} \geq 1$ e $n:=N_{2} \geq 0$. Denotamos ângulos que o primeiro e o último lado do desdobramento formam com o lado 2 por $\varepsilon_{1}$ e $\varepsilon_{2}$. Veja a figura 2.13 .

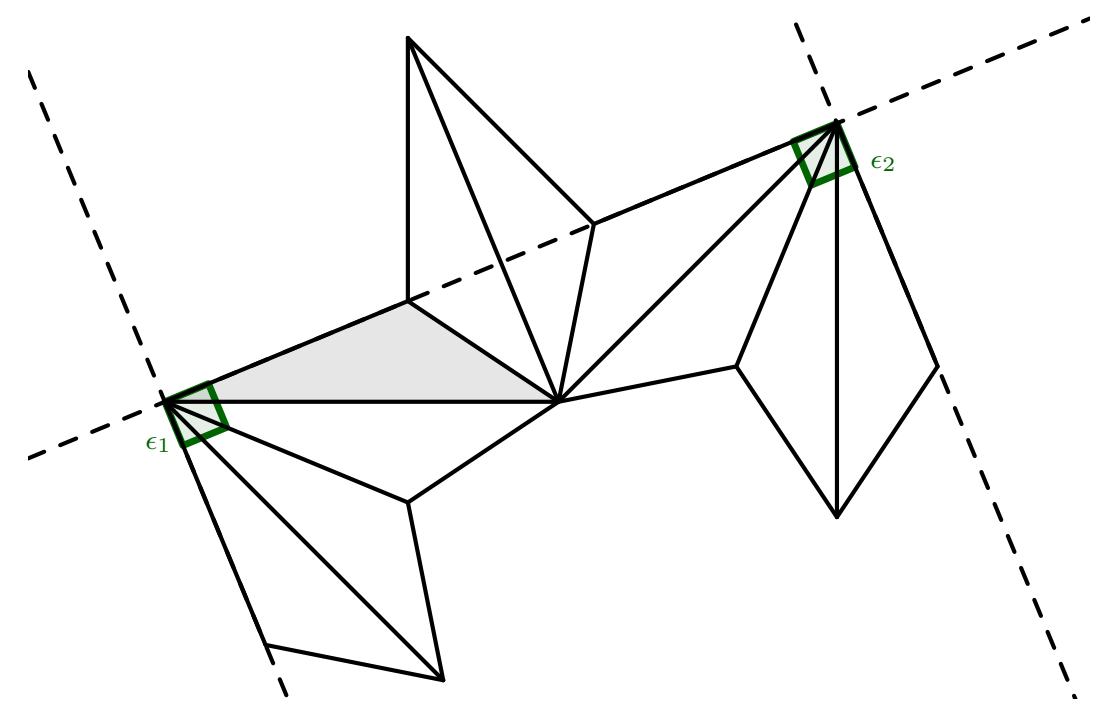

Figura 2.13: $\langle 3|\mathcal{L}| 4,2\rangle$ com $\alpha_{0}$ e $\beta_{0}$ tais que $\varepsilon_{1}=\varepsilon_{2}=\frac{\pi}{2}$.

Note que

$$
\left\{\begin{array}{l}
\varepsilon_{1}=k \alpha \\
\varepsilon_{2}=\pi-2 m \beta+n \alpha
\end{array}\right.
$$


Tomando $\alpha_{0}=\frac{\pi}{2 k}$ e $\beta_{0}=\frac{\pi(k+n)}{4 k m}$, temos $\varepsilon_{1}=\varepsilon_{2}=\frac{\pi}{2}$. Como no caso (a), valores de $\varepsilon_{1}$ e $\varepsilon_{2}$ suficientemente próximos de $\frac{\pi}{2}$ são atingidos com pequenas perturbações de $\alpha_{0}$ e $\beta_{0}$.

Se as condições $2+n<k(2 k-n), 2 k m(2 k+2)<(k+1)(m+1)(2 k+2+n)$ são satisfeitas, então existem $\alpha$ e $\beta$ tais que os segmentos $\overline{P Q}, \overline{Q R}$ e $\overline{R P}$ representam uma órbita periódica no triângulo de ângulos $\alpha$ e $\beta$. Veja a figura 2.14.

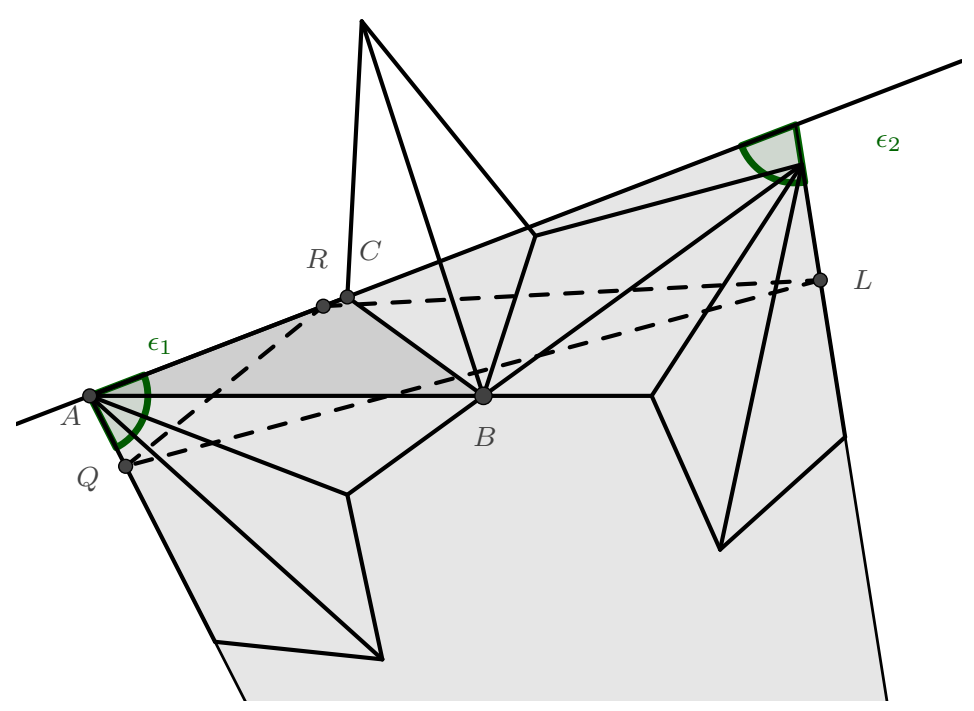

Figura 2.14: $\langle 3|\mathcal{L}| 4,2\rangle$ com $\alpha$ e $\beta$ tais que os segmentos tracejados são uma órbita bilhar periódica.

Para o caso do teorema 2.1.1, a configuração é $\left\langle n_{1}|\mathcal{B}| N_{1}\right\rangle$ ou $\left\langle n_{1}|\mathcal{L}| N_{1}\right\rangle$ e o gerador é $\left\{312(32)^{k-1}(31)^{l-1}: k+l>2\right\}$. Quando $k=l$, temos a família de palavras $Y_{k, 1}$ que aparacerão no teorema 3.1.1.

\subsection{Triângulos obtusos com ângulos menores que $100^{\circ}$}

Nesta seção, citaremos alguns resultados e os principais pontos de suas demonstrações. Para isso, teremos listas de palavras no alfabeto $\{1,2,3\}$ que serão utilizadas apenas nesta seção. No capítulo 3 aparecerão listas de palavras diferentes, no entanto algumas terão o mesmo nome que as aqui listadas.

Em [VGS92], os autores mostram na seção 12 o seguinte teorema.

Teorema 2.3.1. Todas as órbitas bilhares periódicas num triângulo de ângulos $30^{\circ}, 60^{\circ}$ e $90^{\circ}$ são instáveis.

Mais geralmente, veremos na seção 4.1 que todas as órbitas bilhares periódicas em triângulos retângulos são instáveis. Logo, os pontos que representam os triângulos retângulos não estão contidos no interior de nenhum ladrilho de órbita e podemos imaginar que seria difícil cobrir uma vizinhança obtusa dos triângulos retângulos com ladrilhos de órbita. Felizmente, Richard Evan Schwartz [Sch09] mostra que

Teorema 2.3.2. Todo triângulo obtuso cujos ângulos são menores que $100^{\circ}$ possui órbita bilhar periódica estável.

A demonstração deste teorema é feita em várias etapas, parte delas com "matemática tradicional" e outra parte auxiliada por computação ${ }^{3}$. A seguir, descreveremos estes passos. Para essa

\footnotetext{
${ }^{3}$ Em 2006, Richard Evan Schwartz e William Patrick Hooper desenvolveram um programa em Java chamado
} 
demonstração, trabalharemos apenas com uma metade de $\Delta$, com $\beta \geq \alpha$, já que a existência de órbitas periódicas em um triângulo $(\alpha, \beta)$ implica na existência de órbitas bilhares periódicas em um triângulo $(\beta, \alpha)$, pois um é a reflexão do outro e basta trocar os símbolos da palavra que representa a órbita.

Um "ponto problema" na demonstração deste resultado é justamente ${ }^{4}$ o $\left(\frac{\pi}{6}, \frac{\pi}{3}\right)$, pois, em [Sch06], temos os seguintes teoremas.

Teorema 2.3.3. Seja $S_{\epsilon}=\left\{(\alpha, \beta) \in \Delta: \max \left(\left|\alpha-\frac{\pi}{3}\right|,\left|\beta-\frac{\pi}{6}\right|\right)<\epsilon\right\}$. Para todo $\epsilon>0$, existe um triângulo em $S_{\epsilon}$ que não possui órbita bilhar periódica com menos de $\frac{1}{\epsilon}$ segmentos.

Teorema 2.3.4. Todo triângulo em $S_{1 / 400}$ possui órbita bilhar periódica.

Ou seja, precisaríamos de infinitos ladrilhos de órbita para cobrir qualquer vizinhança de $\left(\frac{\pi}{6}, \frac{\pi}{3}\right)$, mas mesmo assim conseguimos fazer isso e mostrar que todo ponto próximo tem órbita bilhar periódica.

Para demonstrar o teorema 2.3.2, basta cobrir $I_{100}:=\left\{(\alpha, \beta): \alpha+\beta>\frac{4 \pi}{9}\right\} \in \Delta-$ que é o conjunto dos triângulos obtusos com todos os ângulos menores que $\frac{5 \pi}{9}=100^{\circ}-$ com ladrilhos de órbita.

Já sabemos dois problemas que serão enfrentados nesta demonstração. Um deles, que já citamos, é uma vizinhança do ponto $\left(\frac{\pi}{6}, \frac{\pi}{3}\right)$, que precisará de infinitos ladrilhos de órbita segundo o teorema 2.3.3. Outro é com respeito a triângulos próximos do segmento $\alpha=0$, pois podemos mostrar de maneira semelhante a da proposição 0.0.1 que todas as órbitas periódicas em pontos suficientemente próximos de um ponto $(0, \beta)$ tem mais de $n$ segmentos. Mais precisamente, em [Sch09] o autor trabalha com a região $P_{3} \in \Delta$ que é o triângulo no espaço dos parâmetros com coordenadas $\left(0, \frac{\pi}{2}\right)$, $\left(0, \frac{3 \pi}{8}\right)$ e $\left(\frac{\pi}{8}, \frac{3 \pi}{8}\right)$. Para cobrir $P_{3}$ serão necessários infinitos ladrilhos de órbita.

Assim, os passos (ilustrados na figura 2.15) para a demonstração de 2.3.2 são

1. Encontrar uma união infinita de ladrilhos de órbita que cubra a região $P_{3}$;

2. Encontrar uma união infinita de ladrilhos de órbita que cubra $P_{6}=S_{1 / 400}$ (teorema 2.3.4);

3. Cobrir uma vizinhança $P_{4}$ do ponto $\left(\frac{\pi}{4}, \frac{\pi}{4}\right)$ com uma união de nove ladrilhos de órbita;

4. Cobrir uma vizinhança $P_{5}$ do ponto $\left(\frac{\pi}{5}, \frac{3 \pi}{10}\right)$ com uma união de dois ladrilhos de órbita;

5. Cobrir $I_{100} \backslash\left(P_{3} \cup S_{1 / 400} \cup P_{4} \cup P_{5}\right)$ com uma união de 215 ladrilhos de órbita.

Os itens 1 e 2 são demonstrados utilizando técnicas analíticas para mostrar que certas famílias infinitas de palavras representam órbitas bilhares periódicas em alguns triângulos da região de interesse. Tais técnicas, apesar de serem "matemática tradicional", foram inspiradas por observações computacionais por parte do autor.

Especificamente, o item 1 usa as famílias de palavras $A_{n}=31(23)^{n+1} 1(23)^{2 n+1} 21(32)^{n+1} 1$ e $B_{n}=31(23)^{n+2} 1(23)^{2 n+2} 21(23)^{n+1} 1$. Veja a seção 3 de [Sch09] para a demonstração.

McBilliards (disponível em: https://sourceforge.net/projects/mcbilliards/) para simular as órbitas bilhares e calcular os ladrilhos de órbita. Esta verão do programa foi descontinuada e Hooper disponibilizou uma nova versão, chamada McBilliards II (disponível em: http://wphooper.com/visual/mcb2/).

${ }^{4}$ Todos os resultados valem, como já dito, para o triângulo $\left(\frac{\pi}{3}, \frac{\pi}{6}\right)$ que é simétrico, porém para simplificar os resultados enunciados vamos citar apenas um dos "lados" simétricos com relação ao segmento $\alpha=\beta$. 


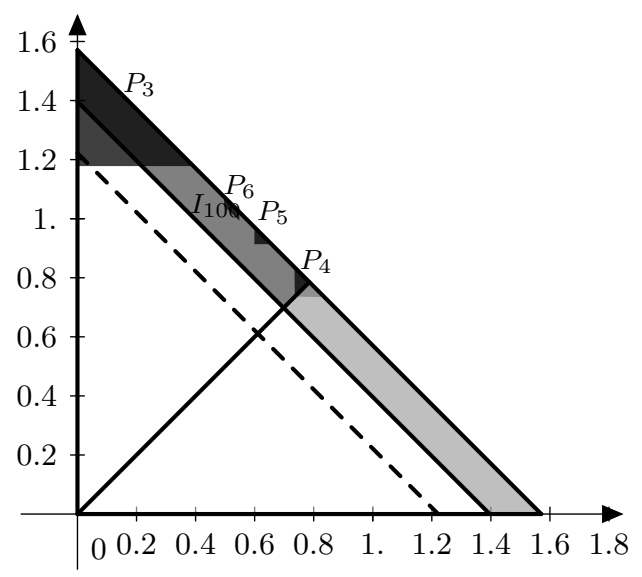

Figura 2.15: Regiões no espaço dos parâmetros abordadas nesta seção. A linha tracejada delimita os triângulos cujo maior ângulo é menor que $110^{\circ}$, que Schwartz afirma que com um pouco mais de trabalho, também é possivel cobrir. Os resultados aqui são para a região $I_{100}$ dos triângulos cujo maior ângulo é menor que $100^{\circ}$.

O item 2 é demonstrado utilizando principalmente as famílias de palavras $\left\{Y_{k}\right\}$ e $\left\{Z_{k}\right\}$. Tais palavras possuem a propriedade de ter desdobramentos simétricos, por isso representamos graficamente apenas metade e desenhamos uma linha tracejada de simetria nas figuras 2.16, 2.17, 2.18 e 2.19, que são os desdobramentos de $Y_{1}, Y_{2}, Y_{3}$ e $Y_{4}$.

As palavras $Y_{k}$ são da forma $Y_{k}=y_{k} 2 y_{k}^{-1} 2$, onde $\omega^{-1}$ é a palavra $\omega$ ao contrário. É desta estrutura que vem a simetria acima citada. As palavras $Z_{k}$ são da forma $Z_{k}=z_{k} 3 z_{k}^{-1} 3$. Temos

$$
\begin{aligned}
y_{2 k+1} & =312323213(2312323213)^{k} 213123(23213)^{2 k}, \\
y_{2 k+2} & =312323213(2312323213)^{k} 123123(2321323123)^{k+1}, \\
z_{k} & =123231323 H_{k} 12323132,
\end{aligned}
$$

com $H_{1}=(213)(213), H_{2}=(213)(123)(123)(213), H_{3}=(213)(123)(213)(213)(123)(213), H_{4}=$ $(213)(123)(213)(123)(123)(213)(123)(213)$ e, mais geralmente, $H_{k}=(213)(123) \ldots(123)(213)$ onde o número de blocos de 3 símbolos é $2 k$. Veja [Sch06] para mais detalhes.

Os itens 3, 4 e 5 são demonstrados em [Sch09] utilizando assistência de computador. Schwartz trabalha com polígonos de vértices racionais diádicos ${ }^{5}$ contidos em cada ladrilho de órbita de interesse. Dada a palavra $W_{k}$, o autor associa um polígono com vértices racionais diádicos $P_{k}$ e cria um método combinatório com finitos passos para verificar se $P_{k} \subset O\left(W_{K}\right)$. Este método é aplicado às 215 palavras do item 5 e às palavras dos itens 3 e 4 .

Este método tem finitos passos pois os ladrilhos de órbita tem finitos "lados" e a lista de palavras para ser analisada é finita. Neste passo, são usadas as funções comparadoras que introduziremos e usaremos no capítulo 3. Veja a seção 6 de [Sch09] para detalhes computacionais.

Para cobrir $P_{4}$ no item 3 , usamos nove ladrilhos de órbita. Como $\left(\frac{\pi}{4}, \frac{\pi}{4}\right)$ está no eixo de simetria $\alpha=\beta$ do espaço dos parâmetros, na verdade precisamos de cinco ladrilhos de órbita: um deles conterá um trecho do segmento $\alpha=\beta$ e os outros quatro podem ser refletidos para gerar os ladrilhos restantes ${ }^{6}$. Veja a figura 2.20.

\footnotetext{
${ }^{5}$ Racionais diádicos são irredutíveis com denominador potência de 2.

${ }^{6}$ Dada uma palavra $\omega$, ao substituir os 1's por 2's e os 2's por 1's, obtemos uma nova palavra $\hat{\omega}$. Os ladrilhos $O(\omega)$ e $O(\hat{\omega})$ são simétricas com relação à reta $\alpha=\beta$.
} 


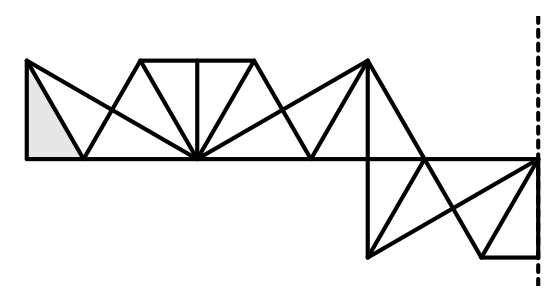

Figura 2.16: $Y_{1}$.

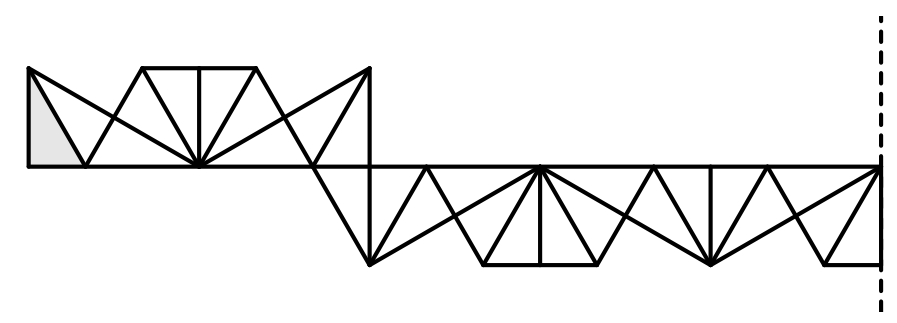

Figura 2.17: $Y_{2}$.

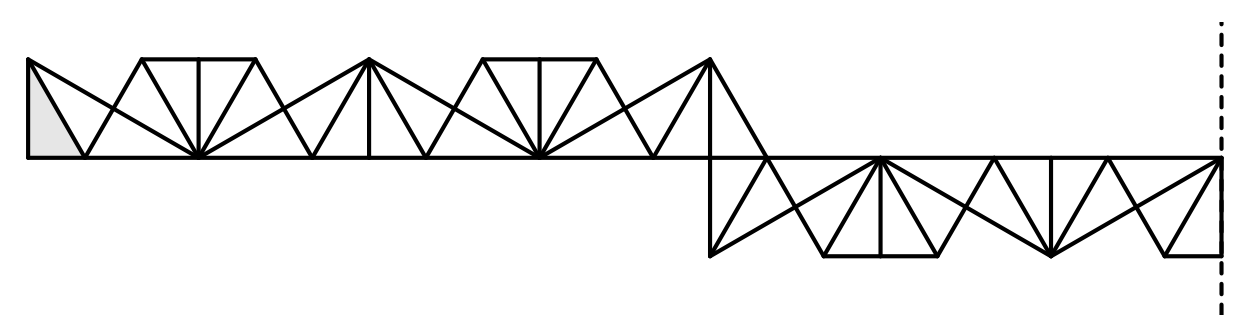

Figura 2.18: $Y_{3}$.

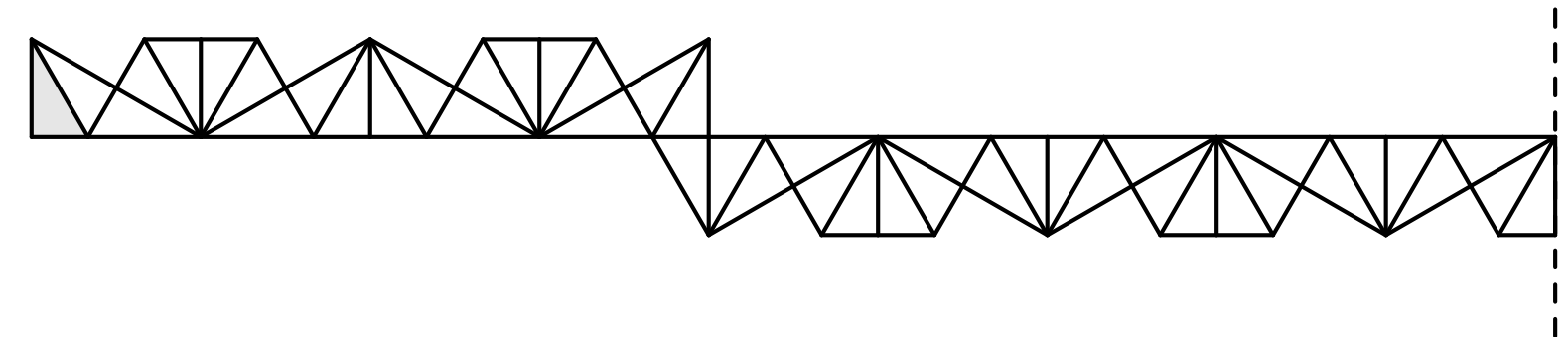

Figura 2.19: $Y_{4}$.

As palavras usadas são

$$
\begin{aligned}
C & =(1232313)^{2} \\
D_{1} & =231323123231323123232132313232132313 \\
D_{2} & =2313231323123231323132312323213231323132321323132313 \\
E_{1} & =12323132312323213231323132321323132313231323 \\
E_{2} & =123231323132312323213231323132313232132313231323132313231323 .
\end{aligned}
$$

Para cobrir $P_{5}$ no item 4, usamos dois ladrilhos de órbita definidos pelas palavras

$$
\begin{aligned}
& F=3123231312313232313213132321 \\
& G=132312323132321321312323132321312312323132321323
\end{aligned}
$$




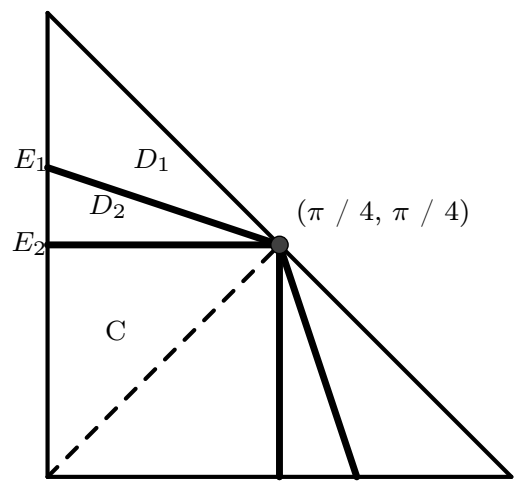

Figura 2.20: Os ladrilhos de órbita das palavras $D_{1}$ e $D_{2}$ cobrem as regiões triangulares abertas indicadas. $O(C)$ cobre o quadrado aberto e $O\left(E_{1}\right)$ e $O\left(E_{2}\right)$ cobrem os segmentos mais escuros indicados. 


\section{Capítulo 3}

\section{Bilhares em triângulos obtusos quase isósceles}

Hooper e Schwartz mostram em [HS09] o seguinte

Teorema 3.0.1. Qualquer perturbação suficientemente pequena de um triângulo isósceles possui órbita bilhar periódica.

A demonstração deste teorema envolve cobrir o segmento aberto $I_{s}=\{(\alpha, \beta) \in \Delta: \alpha=\beta\}$ de triângulos obtusos com famílias infinitas de ladrilhos de órbita. Lembre que um ladrilho de órbita $O(W)$ é o conjunto dos triângulos no espaço dos parâmetros nos quais a palavra $W$ codifica uma órbita periódica.

Exemplo 3.0.1. O triângulo $V_{3}=\left(\frac{\pi}{6}, \frac{\pi}{6}\right)$ possui a órbita 2323132313123232313131, que pelo teorema 2.0.1 é estável (veja a figura 3.1). Logo $V_{3} \in O(2323132313123232313131)$.

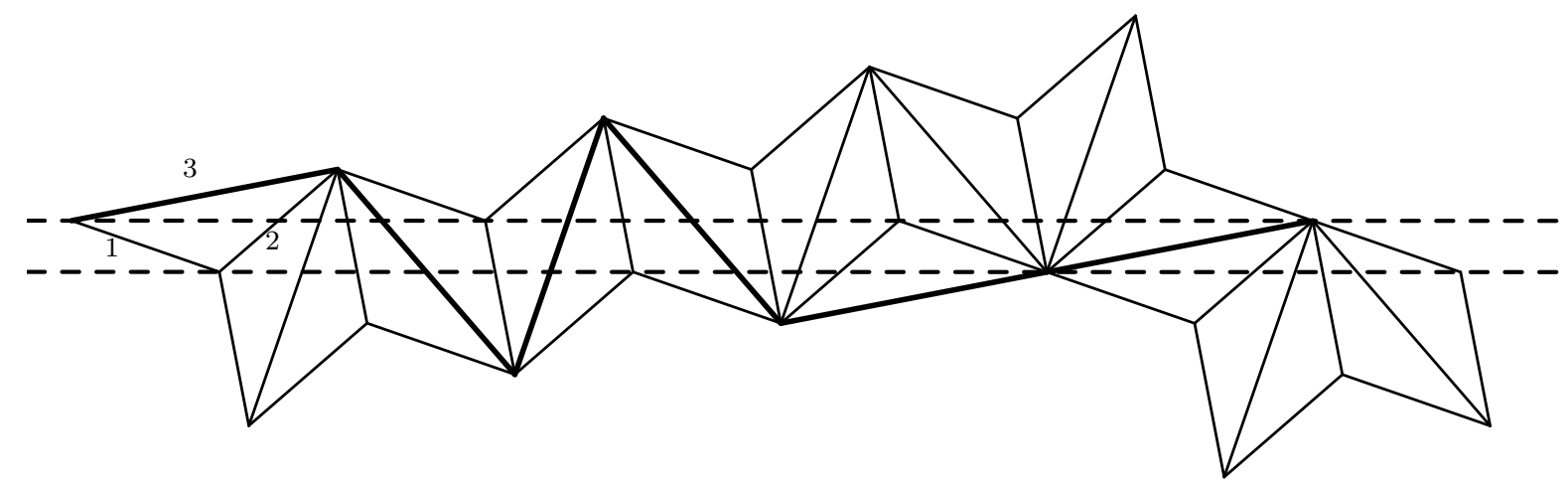

Figura 3.1: Desdobramento da palavra 2323132313123232313131 no triângulo $\left(\frac{\pi}{6}, \frac{\pi}{6}\right)$.

Um triângulo de Veech ${ }^{1}$ é um triângulo isósceles obtuso cujos ângulos agudos medem $\frac{\pi}{2 n}$ para algum $n \in \mathbb{N}$. Como o triângulo é obtuso, $n$ deve ser maior ou igual a 3. Usando a representação dos triângulos no espaço de parâmetros $\Delta$, um triângulo de Veech é $V_{n}=\left(\frac{\pi}{2 n}, \frac{\pi}{2 n}\right), n=3,4,5, \ldots$

O teorema a seguir, que abordaremos na seção 4.2, mostra o motivo da dificuldade que teremos em demonstrar o teorema 3.0.1.

Teorema 3.0.2. O triângulo $V_{2^{k}}=\left(\frac{\pi}{2^{k+1}}, \frac{\pi}{2^{k+1}}\right)$, com $k=3,4,5, \ldots$, não está contido no interior de nenhum ladrilho de órbita.

Se $n \geq 3$ não é uma potência de dois, $V_{n}$ está contido em um ladrilho de órbita.

\footnotetext{
${ }^{1} \mathrm{O}$ motivo deste nome será melhor abordado no capítulo 4 e se refere William A Veech por [Vee89].
} 
A seguir, vamos enunciar e demonstrar três teoremas (3.1.1, 3.2.1 e 3.3.1) que juntos cobrem todos os casos além do exemplo 3.0.1 e do caso $\left(\frac{\pi}{4}, \frac{\pi}{4}\right)$, que está contemplado na seção 2.3. Assim, o teorema 3.0.1 estará provado. Antes de cada teorema, teremos que introduzir alguns conceitos e resultados auxilares, por isso dividiremos cada um deles em uma seção.

Esses resultados, juntamente com as classes de órbitas ímpares em [HH00] e os triângulos com ângulos menores que $100^{\circ}$ cobrem uma grande região do espaço dos parâmetros, porém a questão sobre todo triângulo possuir órbita bilhar periódica continua em aberto.

\subsection{As famílias de palavras $W_{n}$ e $Y_{n, m}$}

Nesta seção, definimos as famílias de palavras

$$
W_{n}=(31)^{n-1}(32)^{n-1} \text { e } Y_{n, m}=1\left(W_{n}\right)^{m} 32
$$

e mostraremos que as palavras $Y_{n, m}$ definem órbitas periódicas em alguns triângulos isósceles. Como $Y_{n, m}$ é ímpar, $Y_{n, m}^{2}$ é estável e isso definirá uma vizinhança desses triângulos onde a órbita se mantém.

Denotaremos por $U\left(W, T_{X}\right)$ ou $U(W, X)$ o desdobramento da órbita codificada pela palavra $W$ no triângulo representado pelo par de ângulos $X=(\alpha, \beta)$. W representará um órbita periódica em $T_{X}$ se o primeiro e o último lado do desdobramento forem paralelos e existir um segmento contido no desdobramento ligando os dois lados em pontos equivalentes, sem passar por nenhum vértice.

Podemos sempre rotacionar a figura de modo que o primeiro e o último lado estejam relacionados por uma translação horizontal, chamada holonomia ${ }^{2}$. Feito isso, dizemos que os vértices superiores são os que percorrem a parte superior do desdobramento e os vértices inferiores percorrem a parte inferior. Denotaremos por $a_{k}$ e $b_{k}, k=1,2,3 \ldots$, os vértices superiores e inferiores, respectivamente.

Se não existe sobreposição dos triângulos no desdobramento, um vértice é superior se existe uma direção com coordenada $y$ positiva tal que o raio partindo do vértice nesta direção nunca intercepta o desdobramento e um vértice é inferior se existe uma direção com coordenada $y$ negativa tal que o raio partindo do vértice nesta direção nunca intercepta o desdobramento.

Na figura 3.1, por exemplo, o vértices superiores $a_{6}$ e $a_{8}$ coincidem e $a_{7}$ está no meio de um triângulo equilátero de lado 3 . O segmento $\overline{a_{6} a_{7}}$ não é uma linha de reflexão do desdobramento.

Definição 3.1.1. O desdobramento de uma palavra da forma $(i j)^{k-1} i$, com $i, j \in\{1,2,3\}$ é um $k$-leque. Em outras palavras, é a união de $2 k$ triângulos refletidos ao redor de um vértice comum.

Como estamos trabalhando com triângulos obtusos, vamos nos restringir a leques do tipo $(13)^{k-1} 1$ ou $(23)^{k-1} 2$. Isso faz com que o primeiro e o último lado dos leques seja um lado 3 , e é como vamos particionar nossos desdobramentos em leques maximais. Não nos interessam leques do tipo $(12)^{k-1} 1$ ou $(21)^{k-1} 2$ para $k \geq 2$, pois o ângulo em torno do qual os triângulos estariam refletidos seria maior que $\frac{\pi}{2}$ e não haveria órbita passando por tal leque.

Chamaremos a poligonal formada por arestas do tipo 3 que são os extremos de cada leque maximal de 3-espinha. Ela liga o primeiro ao último lado. Destacamos a 3-espinha na figura 3.1.

Em um leque, dizemos que os vértices do primeiro e do último triângulo são vértices base, e os demais são vértices ponta. Um vértice base especial é o que faz parte de todos os triângulos do leque, chamaremos este de centro. Veja a figura 3.2. O raio que parte do centro e passa pela bissetriz do ângulo formado pelo primeiro e o último lado é a linha central. Um leque aponta para cima se sua linha central aponta para cima e, analogamente aponta para baixo se sua linha central aponta para baixo.

Todo desdobramento pode ser decomposto em leques maximais. Seja $\delta(W)$ o maior $k$ tal que $U(W,$.$) contém um k$-leque. Na figura $3.1, \delta(W)=3$.

\footnotetext{
${ }^{2}$ Ver capítulo 4.
} 


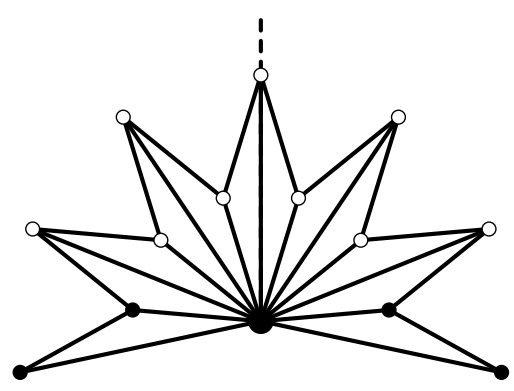

Figura 3.2: Um 6-leque, cuja palavra correspondente é 13131313131. Os vértices brancos são ponta, os pretos são base e o maior dos pretos é o centro do leque.

Lema 2 (Leque $\left.^{3}\right)$. Seja $W$ uma palavra estável e $X=(\alpha, \beta)$ um ponto no espaço dos parâmetros. Suponha que $\max (\alpha, \beta) \leq \frac{\pi}{\delta(W)}$ e que todos os vértices base superiores de $U\left(W, T_{X}\right)$ estão acima de todos os vértices base inferiores de $U\left(W, T_{X}\right)$. Então $X \in O(W)$.

Demonstração. Como $W$ é estável, queremos mostrar que $U\left(W, T_{X}\right)$ possui um segmento ligando o primeiro e o último lado em pontos correspondentes. Rotacionamos o desdobramento de modo que a translação que leva o primeiro lado ao último é horizontal.

Basta mostrar que todo vértice superior está acima de cada vértice inferior. Mas, por hipótese, todos os vértices base superiores estão acima de todos os vértices base inferiores, então resta mostrar que os vértices ponta não vão interferir. Vejamos que o vértice superior mais baixo e o vértice inferior mais alto são vértices base. Faremos o argumento para vértices superiores, e para inferiores é análogo.

Seja $a_{i}$ um vértice ponta superior pertencente a um leque maximal $D$. Queremos mostrar que existe um vértice base superior $a_{j}$ em $D$ tal que $a_{j}$ está abaixo de $a_{i}$. Como $a_{i}$ é superior, então o centro $c_{D}$ de $D$ é um vértice inferior. Além disso os dois vértices base menos externos $i_{1}$ e $i_{2}$ (que são as extremidades do segundo e do penúltimo lado de $D$ ) são também superiores. Logo, como o centro é um vértice base inferior e $i_{1}$ e $i_{2}$ são vértices base superiores, por hipótese, $i_{1}$ e $i_{2}$ estão acima de $c_{D}$. O ângulo entre a linha central e o penúltimo lado é $(k-1) \alpha$ ou $(k-1) \beta$. Pela hipótese $\max (\alpha, \beta) \leq \frac{\pi}{\delta(W)}$, podemos assumir sem perda de generalidade que é $(k-1) \alpha$ e temos que $(k-1) \alpha \leq(k-1) \frac{\pi}{\delta(W)}<\delta(W) \frac{\pi}{\delta(W)}=\pi$. Logo os vetores $\overrightarrow{c_{D} i_{1}}$ e $\overrightarrow{c_{D} i_{2}}$ apontam pra cima e a linha central, que é a bissetriz deles, também aponta pra cima. Logo, $D$ aponta pra cima.

Como o leque é da forma $(13)^{k-1} 1$ ou $(23)^{k-1} 2$, o segundo e o penúltimo lado de $D$ são 1 ou 2 e todos as arestas mais curtas de $D$ tem o mesmo tamanho. Sabemos que $\overrightarrow{c_{D}} \overrightarrow{i_{1}}$ e $\vec{c}_{D} \overrightarrow{i_{2}}$ apontam pra cima e $i_{1} \hat{C_{D}} i_{2}<2 \pi$, então o segmento $\overline{i_{1} i_{2}}$ separa o centro de todos os vértices ponta, portanto os vértices base superiores $i_{1}$ e $i_{2}$ estão abaixo de $a_{i}$.

Teorema 3.1.1. Para todo $n \geq 2$ e $\alpha \in\left(\frac{\pi}{2 n+2}, \frac{\pi}{2 n}\right)$, existe $m \in \mathbb{N}$ tal que $Y_{n, m}$ codifica uma órbita bilhar periódica em $T_{(\alpha, \alpha)}$.

Demonstração. Dividimos esta demonstração em dois casos.

Caso 1: $\alpha \in\left[\frac{\pi}{2 n+1}, \frac{\pi}{2 n}\right)$

Consideramos o desdobramento $U\left(Y_{n, 1}^{2}, T_{(\alpha, \alpha)}\right)$. Queremos mostrar que existe uma órbita bilhar periódica neste desdobramento. Como $Y_{n, 1}^{2}$ é estável, esta órbita será estável.

Primeiro, vejamos que se rotacionarmos o desdobramento de modo que a translação entre o primeiro e o último lado seja horizontal, então o lado $\overline{A B}$ (lado 3) também é horizontal. Veja a

\footnotetext{
${ }^{3} \mathrm{Na}$ referência original, Hooper e Schwartz chamam este de "Dart Lemma", leque de "dart" e os vértices base e ponta de um k-leque de "superior" e "inferior", respectivamente. Optamos por esta adaptação para o português, em primeiro lugar, para evitar confusão com os vértices superiores e inferiores (no original "top" e "bottom") e, em segundo lugar, por transmitir melhor a ideia da configuração do desdobramento.
} 
figura 3.3. Isso segue do fato da palavra $Y_{n, 1}$ ter uma certa simetria e do triângulo ser isósceles. $Y_{n, 1}$ pode ser escrita como $1 W 3 \hat{W} 2$, onde $\hat{W}$ é $W$ ao contrário com 1's e 2's trocados. Então o desdobramento infinito horizontal da palavra ...1W3W $21 W 3 \hat{W} 2 \mid 1 W 3 \hat{W} 21 W 3 \hat{W} 2 \ldots$ em um triângulo isósceles será simétrico com relação a linha vertical indicada. Mas esta linha divide um triângulo isósceles no meio, que é exatamente o triângulo que corresponde ao primeiro triângulo do desdobramento (tracejado na figura 3.3). Logo, como este triângulo isósceles é simétrico com uma linha vertical, seu maior lado é horizontal.

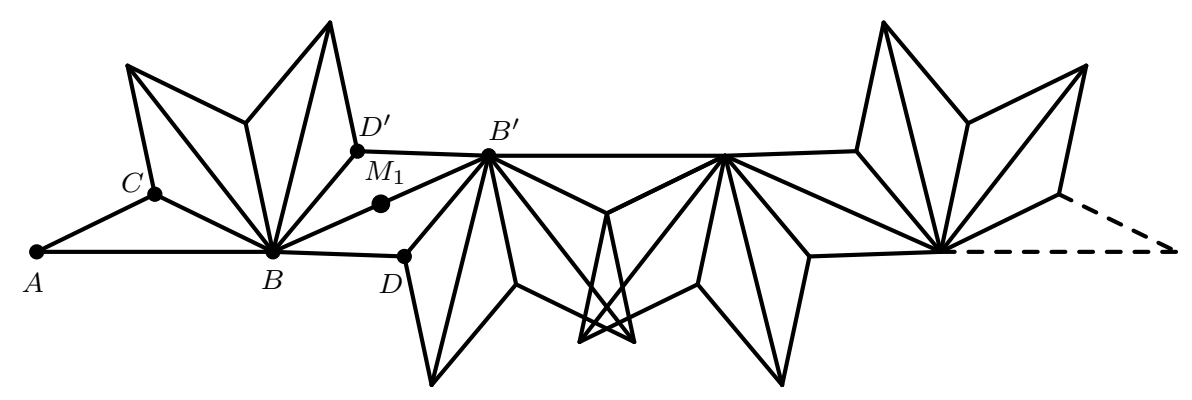

Figura 3.3: Desdobramento do triângulo $T_{(\alpha, \alpha)}$ com relação a palavra $Y_{n, 1}^{2}$, com $n=3$

Fixamos $d(A, B)=1$ e um sistema de coordenadas cartesianas com $M_{1}=(0,0)$, onde $M_{1}$ é o centro do último lado do primeiro leque maximal. Para mostrar que existe um segmento inteiramente contido no desdobramento ligando o lado $\overline{A C}$ (lado 2) ao último lado do desdobramento no ponto correspondente sem passar por nenhum vértice e passando por $M_{1}$, basta mostrar que todos os vértices superiores tem coordenada $y$ positiva e todos os inferiores tem coordenada $y$ negativa.

Note que basta mostrar para os pontos $A, B, C$ e $D$ pelo lema 2 e pela simetria do desdobramento.

Dado um ponto $\mathrm{P}$ no plano cartesiano, denotaremos suas coordenadas como $\left(x_{P}, y_{P}\right)$. Como discutimos acima, o lado 3 é horizontal e $y_{A}=y_{B}$. O ângulo $A \hat{B} B^{\prime}=2 n \alpha<\pi, \operatorname{logo} 0=y_{M_{1}}>$ $y_{A}=y_{B}$. Mais especificamente, $y_{A}=y_{B}=-\frac{\sin (\pi-2 n \alpha)}{2}$.

Ainda, $A \hat{B} D=(2 n+1) \alpha \geq \pi$ implica que $y_{D}<y_{B}<0$.

Agora vejamos que $y_{C}>0$. Temos que:

$$
\tan (\alpha)=\frac{y_{C}-y_{B}}{1 / 2}=2 y_{C}+\sin (\pi-2 n \alpha) \Longrightarrow y_{C}=\frac{\tan (\alpha)-\sin (\pi-2 n \alpha)}{2}
$$

Como $(2 n+1) \alpha \geq \pi$, então $\alpha \geq \pi-2 n \alpha$, e como $2 n \alpha<\pi$, então $0<\pi-2 n \alpha \leq \alpha$. Além disso, temos que $\alpha<\frac{\pi}{2 n}$ e $n \geq 2$, então $\alpha \in\left(0, \frac{\pi}{4}\right)$. Logo, $\sin (\alpha)>\sin (\pi-2 n \alpha)$ e $\tan (\alpha)=\frac{\sin (\alpha)}{\cos (\alpha)}>\sin (\alpha)$. Portanto

$$
\tan (\alpha)-\sin (\pi-2 n \alpha)>\sin (\alpha)-\sin (\alpha)=0 \Longrightarrow y_{C}>0
$$

Caso 2: $\alpha \in\left(\frac{\pi}{2 n+2}, \frac{\pi}{2 n+1}\right)$

Consideremos o desdobramento $U\left(1\left(W_{n}\right)^{\infty}, T_{(\alpha, \alpha)}\right)$, rotacionado de modo que o lado 3 é horizontal. Fixamos os pontos $M_{0}, M_{1}, M_{2}, \ldots$ como pontos médios dos lados 3 onde os $n$-1-leques tem um lado em comum. Mais especificamente, $M_{0}$ é o ponto médio do terceiro lado do desdobramento e $M_{i}$ é o centro do último lado do $i$-ésimo leque maximal, $i \geq 1$. Veja a figura 3.4.

Vamos mostrar que existe $m$ tal que $M_{m}$ está abaixo de todos os vértices superiores e acima de todos os vértices inferiores anteriores a ele. Isso implica que teremos um seguimento horizontal contido no corredor do desdobramento da palavra $1 W 3$ (aqui $W=1\left(W_{n}\right)^{\frac{m}{2}}$ se $m$ é par e $W=$ $1\left(W_{n}\right)^{\frac{m-1}{2}}(31)^{n-1}$ se $m$ é ímpar), que começa no lado 2 e termina em $M_{m}$. Este segmento resultará 


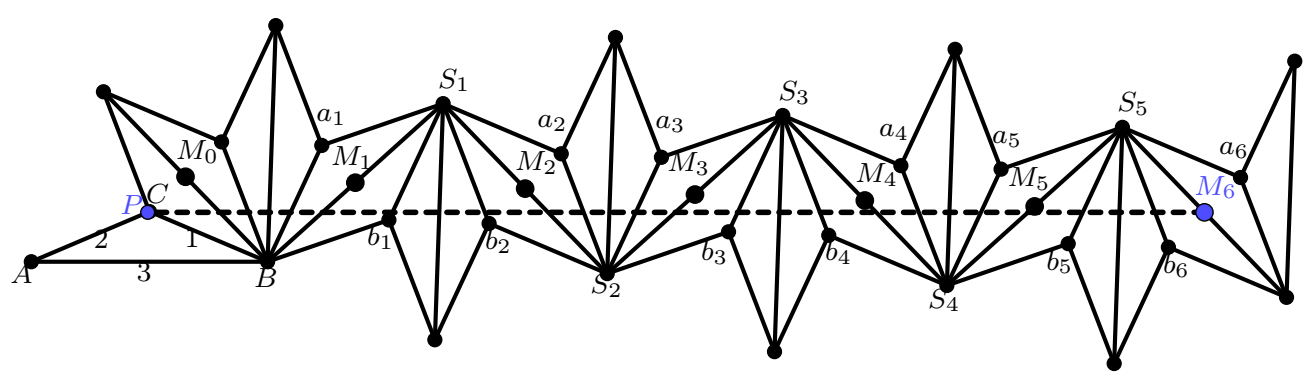

Figura 3.4: Desdobramento do triângulo $T_{(\alpha, \alpha)}$ com relação a palavra $1\left(W_{n}\right)^{\infty}$, com $n=3$. Neste caso, com $\alpha=23^{\circ}, m=6$. O ponto $P$ está abaixo do ponto $C$ e acima do ponto $b_{1}$.

na órbita periódica codificada por $Y_{n, m}=1\left(W_{n}\right)^{m} 32$.

Fixamos a origem em $M_{1}$. Vejamos que $y_{M_{2}}<0$. A inclinação do segmento $\overline{B M_{1}}$ é $\pi-2 n \alpha$. Note que $M_{1} S_{1} M_{2}$ é isósceles e $M_{1} \hat{S}_{1} M_{2}=(2 n-2) \alpha$. Logo, $M_{2} \hat{M}_{1} S_{1}=\frac{\pi-(2 n-2) \alpha}{2}=\frac{\pi}{2}-(n-1) \alpha$. Portanto, a inclinação de $\overline{M_{1} M_{2}}$ com relação à horizontal é

$$
\pi-2 n \alpha-\frac{\pi}{2}+(n-1) \alpha=\frac{\pi}{2}-(n+1) \alpha<0,
$$

pois $\alpha>\frac{\pi}{2 n+2}$ implica $(n+1) \alpha>\frac{\pi}{2}$. Logo $y_{M_{2}}<y_{M_{1}}=0$.

Como $A \hat{B} b_{1}=2 n \alpha+\alpha=(2 n+1) \alpha<\pi$, então $y_{B}<y_{b_{1}}$. Além disso, como $\overline{b_{1} b_{2}}$ é paralelo a $\overline{M_{1} M_{2}}$, então $y_{b_{2}}<y_{b_{1}}$. Mais geralmente, $y_{b_{k+1}}<y_{b_{k}}$ para todo $k \geq 1$. Logo, $b_{1}$ é o vértice inferior com maior coordenada $y$.

Do mesmo modo, como $\overline{a_{1} a_{2}}$ é paralelo a $\overline{M_{1} M_{2}}, y_{a_{2}}<y_{a_{1}}$. Mais geralmente, $y_{a_{k+1}}<y_{a_{k}}$ para todo $k \geq 1$. Note, ainda, que a inclinação de $\overline{a_{1} S_{1}}$ com a horizontal é $\pi-(2 n+1) \alpha>0$, logo $y_{a_{2 k+1}}<y_{S_{2 k+1}}$ para todo $k \geq 0$. Logo, o vértice superior com menor coordenada $y$ até $a_{m}$ é o próprio $a_{m}$ ou $C$.

Portanto, para mostrar que existe $\mathrm{m}$ tal que há um segmento horizontal inteiramente contido no desdobramento ligando $M_{m}$ ao lado $\overline{A C}$, basta mostrar que existe $m$ tal que

$$
y_{C}>y_{M_{m}}>y_{b_{1}} \text { e } y_{M_{m}}<y_{a_{m}} .
$$

Se $m$ é par, $\overline{M_{m} a_{m}}$ tem inclinação $\frac{\pi}{2}-2 \alpha>0$, pois $\overline{S_{m-1} M_{m}}$ tem inclinação $-2 \alpha$ e $\overline{M_{m} a_{m}}$ é a altura do triângulo isósceles. Logo, $y_{a_{m}}>y_{M_{m}}$.

Se $m$ é ímpar, $\overline{M_{m} a_{m}}$ tem inclinação $\pi-2 n \alpha+\frac{\pi}{2}=\frac{3 \pi}{2}-2 n \alpha>\frac{\pi}{2}$, pois $2 n \alpha<\pi$. Logo, $y_{a_{m}}>y_{M_{m}}$.

Vejamos, por fim, que existe $m$ tal que $y_{C}>y_{M_{m}}>y_{b_{1}}$. Primeiro, note que $y_{C}<y_{M_{0}}$ uma vez que a inclinação de $\overline{C M_{0}}=\pi-2 \alpha+\frac{p i}{2}=\frac{\pi}{2}-2 \alpha>0$. Se mostrarmos que

$$
(I)=y_{C}-y_{b_{1}}>y_{M_{k-1}}-y_{M_{k}}=(I I),
$$

então existirá um $m$ tal que $y_{C}>y_{M_{m}}>y_{b_{1}}$.

Para calcular $(I)$ e $(I I)$, vou fixar a origem em $B$, como são distâncias, isso não altera os resultados.

Primeiro, fixado o comprimento unitário para o lado 3, temos que o comprimento dos lados 1 e 2 é $\frac{1}{2 \cos (\alpha)}$. Logo, $y_{C}=\frac{\sin (\alpha)}{2 \cos (\alpha)}$. Como o $\angle A B b_{1}$ mede $(2 n+1) \alpha, y_{b_{1}}=\frac{\sin ((2 n+1) \alpha)}{2 \cos (\alpha)}$. Portanto,

$$
(I)=\frac{\sin (\alpha)-\sin ((2 n+1) \alpha)}{2 \cos (\alpha)} .
$$


Como $\angle A B M_{1}=2 n \alpha$, então $y_{M_{1}}=\frac{\sin (2 n \alpha)}{2}$ e $y_{S_{1}}=\sin (2 n \alpha)$. Uma vez que a inclinação de $\overline{S_{1} M_{2}}$ com a horizontal é $-2 \alpha$, temos que $\sin (2 \alpha)=\frac{y_{S_{1}}-y_{M_{2}}}{1 / 2}$. Logo, $y_{M_{2}}=\sin (2 n \alpha)-\frac{\sin (2 \alpha)}{2}$ o que implica

$$
(I I)=\frac{\sin (2 \alpha)-\sin (2 n \alpha)}{2} .
$$

Agora, aplicamos a $(I)$ e $(I I)$ a seguinte identidade trigonométrica

$$
\sin (\theta)-\sin (\alpha)=2 \sin \left(\frac{\theta-\alpha}{2}\right) \cos \left(\frac{\theta+\alpha}{2}\right) .
$$

Isso nos dá

$$
(I)=-\frac{\sin (n \alpha) \cos ((n+1) \alpha)}{\cos (\alpha)} \text { e }(I I)=-\sin ((n-1) \alpha) \cos ((n+1) \alpha) .
$$

Queremos mostrar que $(I)>(I I)$. Mas

$$
\frac{(I)}{(I I)}=\frac{\sin (n \alpha)}{\sin ((n-1) \alpha) \cos (\alpha)}>\frac{1}{\cos (\alpha)}>1,
$$

uma vez que $0<(n-1) \alpha<n \alpha<\frac{\pi}{2}$ e $0<\alpha<\frac{\pi}{2}$.

O teorema que acabamos de demonstrar garante que todos os pontos de $I_{s}$ no espaço de parâmetros que não são da forma $V_{n}=\left(\frac{\pi}{2 n}, \frac{\pi}{2 n}\right)$ estão contidos num ladrilho de órbita. Veja uma ilustração desses ladrilhos na figura 3.12. A seguir, temos que considerar o caso complicado de cobrir uma vizinhança de $V_{n}$ para $n>3$.

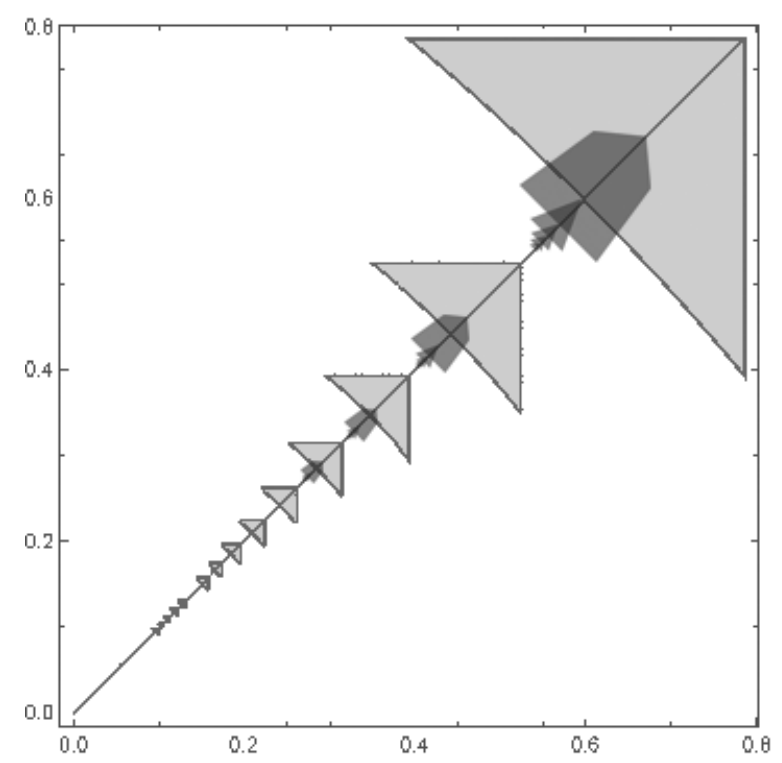

Figura 3.5: Ilustração dos ladrilhos de órbita do teorema 3.1.1. Os mais claros são para o caso 1 (se acumulam no ponto $(0,0)$ ) e os mais escuros são para o caso 2 (se acumulam em pontos da forma $\left(\frac{\pi}{2 n}, \frac{\pi}{2 n}\right)$ para $n=3,4,5, \ldots)$. 


\subsection{As famílias de palavras $A_{n}, B_{n}$ e $C_{n}$}

Em [HS09], os autores usam ladrilhamentos do plano por polígonos para representar órbitas bilhares periódicas em triângulos como caminhos nestes ladrilhamentos. Cada lado desses polígonos tem centro em um ponto pertencente ao reticulado $\mathbb{Z}^{2}$, logo uma órbita é uma sequência de pontos deste reticulado.

Usando estes pontos, é possível calcular funções que nos dão propriedades interessantes da órbita, como a posição de um vértice do desdobramento com relação a outro vértice. Vamos definir as órbitas e estas funções nas próximas seções e elas serão usadas nas demonstrações dos teoremas 3.2.1 e 3.3.1.

\subsection{1 Órbita reticulada hexagonal}

Seja $H_{0}$ um hexágono cujos centros dos lados são os pontos $(0,0),(1,1),(2,1),(2,0),(1,-1)$ e $(0,-1)$ e seus lados opostos são paralelos. Denotamos seus lados por 1, 2 e 3, com lados paralelos tendo a mesma numeração, como na figura 3.6. $\mathcal{H}$ é o ladrilhamento de $\mathbb{R}^{2}$ por $H_{0}$.

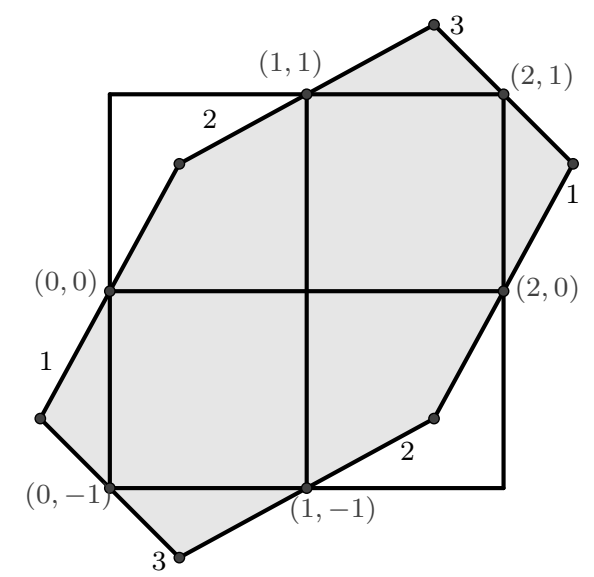

Figura 3.6: É possivel ladrilhar o plano com este hexágono e os prontos médios de suas arestas são $\mathbb{Z}^{2}$.

Como cada vértice deste ladrilhamento está ligado a três arestas, cada uma com um dos números 1, 2 e 3, dada uma palavra $\omega$, podemos partir de um vértice qualquer de $\mathcal{H}$ e percorrer a sequência de arestas codificada por esta palavra. Portanto, fixado o ponto inicial, este caminho é único. Além disso, se a palavra $\omega$ codifica uma órbita bilhar, o caminho percorrido nunca volta pelo mesmo vértice, pois não existem símbolos repetidos consecutivos em $\omega$. Veja a figura 3.7. Chamamos este caminho de órbita reticulada hexagonal e denotamos por $H(\omega)$. Note que mudar o ponto inicial apenas desloca a figura, não mudando sua forma ou orientação, logo não nos preocuparemos em inserir este ponto na notação.

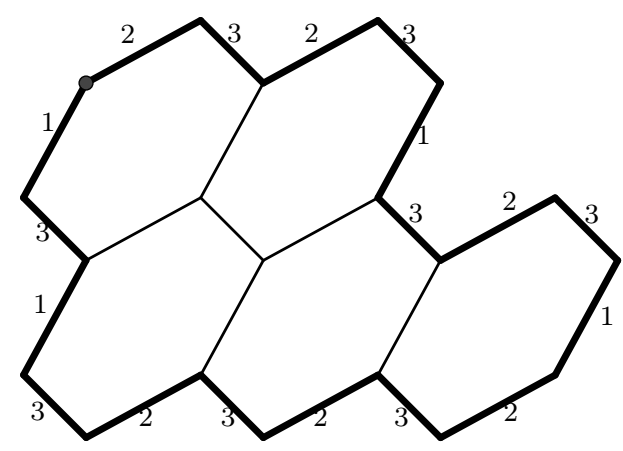

Figura 3.7: Órbita reticulada hexagonal definida pela palavra 232313231232323131. 
Lema 3. A palavra $\omega$ é estável se, e somente se, $H(\omega)$ é um caminho fechado.

Demonstração. Vejamos que $H(\omega)$ ser um caminho fechado é equivalente a cada símbolo de $\omega$ aparecer o mesmo número de vezes em posição par e ímpar, então concluímos o lema pelo teorema 2.0.1.

Sejam $x$ e $y$ os ângulos internos do hexágono $H_{0}$, com $x$ sendo o menor ângulo e $y$ o maior. Seja $N$ par o comprimento de $\omega=\omega_{1} \ldots \omega_{N}$.

Colocamos coordenadas cartesianas no plano, fixando a origem no ponto inicial de $H(\omega)$. Vamos dividir os vértices de $\mathcal{H}$ em dois tipos: o tipo (a) tem um lado 3 a um ângulo de $\alpha_{3}=-\frac{\pi}{4}$, um lado 1 a um ângulo de $\alpha_{1}=x-\frac{\pi}{4}$ e um lado 2 a um ângulo de $\alpha_{2}=x+y-\frac{\pi}{4}$; o tipo (b) tem $\beta_{3}=\frac{3 \pi}{4}$, $\beta_{2}=\frac{3 \pi}{4}-x$ e $\beta_{1}=\frac{3 \pi}{4}+x$. Sair de um vértice (a) por qualquer de suas arestas leva a um vértice (b) e, do mesmo modo, sair de um vértice (b) sempre leva a um vértice (a).

Sejam $p_{1}, p_{2}$ e $p_{3}$ as coordenadas no plano cartesiano da extremidade das arestas 1,2 e 3 , respectivamente, partindo da origem de um vértice de tipo (a). Defino $q_{1}, q_{2}$ e $q_{3}$ como as mesmas coordenadas quando a origem é um vértice do tipo (b). Chamando os comprimentos dos lados $1 \mathrm{e}$ 2 de $l_{1}=l_{2}=l$ e do lado 3 de $l_{3}$, temos:

$$
\begin{aligned}
& p_{3}=l_{3}\left(\frac{\sqrt{2}}{2},-\frac{\sqrt{2}}{2}\right) \\
& p_{1}=l_{1}\left(\cos \left(x-\frac{\pi}{4}\right), \sin \left(x-\frac{\pi}{4}\right)\right) \\
& p_{2}=l_{2}\left(\cos \left(x+y-\frac{\pi}{4}\right), \sin \left(x+y-\frac{\pi}{4}\right)\right)
\end{aligned}
$$

Note que $q_{3}=-p_{3}, q_{2}=-p_{2}$ e $q_{1}=-p_{1}$.

Logo, partindo de um vértice de tipo (a) e percorrendo o caminho $H(\omega)$, chegamos a posição

$$
\sum_{j=1}^{N}(-1)^{j+1} p_{\omega_{j}} .
$$

É imediato que $\omega$ ser estável implica que esta soma é $(0,0)$.

Se esta soma for $(0,0)$, supomos que $\omega$ não é uma palavra estável. Então existiriam $k_{1}, k_{2}$ e $k_{3}$ inteiros não todos nulos tais que $k_{1} p_{1}+k_{2} p_{2}+k_{3} p_{3}=0$, com $k_{1}+k_{2}+k_{3}=0$, o que não é possível com os valores de $p_{1}, p_{2}$ e $p_{3}$.

De fato, o caso em que apenas um $k_{i}$ é diferente de 0 pode ser descartado pela condição $k_{1}+$ $k_{2}+k_{3}=0$. O caso em que dois deles são diferentes de 0 também pois $p_{1}, p_{2}$ e $p_{3}$ são dois a dois linearmente independentes. No caso em que nenhum dos $k_{i}$ 's é diferente de 0 , teríamos $k_{3} p_{3}=k_{1} p_{1}+k_{2} p_{2}$. Mas também não é possível parar na direção $-\frac{\pi}{4}$ só passando por lados 1 e 2 , já que eles tem que ser alternados. Logo, $\omega$ é estável.

Partir de um vértice do tipo (b) apenas muda o sinal da soma, e o lema está provado.

\subsection{2 Órbita reticulada quadrada}

Outra maneira de representar uma órbita bilhar periódica como um caminho nas arestas de um ladrilhamento de $\mathbb{R}^{2}$ é com um quadrado $2 \times 2$. Dada a órbita reticulada hexagonal $H(\omega)$, em cada vértice existe uma aresta 3 adjacente. Para obter a órbita reticulada quadrada, denotada por $\hat{Q}_{3}(\omega)$, basta conectar as arestas 3 ligadas a cada vértice na ordem em que eles aparecem em $H(\omega)$. É possível fazer a mesma definição usando as arestas 1 e 2 , obtendo assim $\hat{Q}_{1}(\omega)$ e $\hat{Q}_{2}(\omega)$, porém vamos fixar agora $\hat{Q}_{3}(\omega)$ que será usada majoritariamente neste trabalho.

Denotaremos por $\mathcal{Q}$ o ladrilhamento do plano por estes quadrados. 


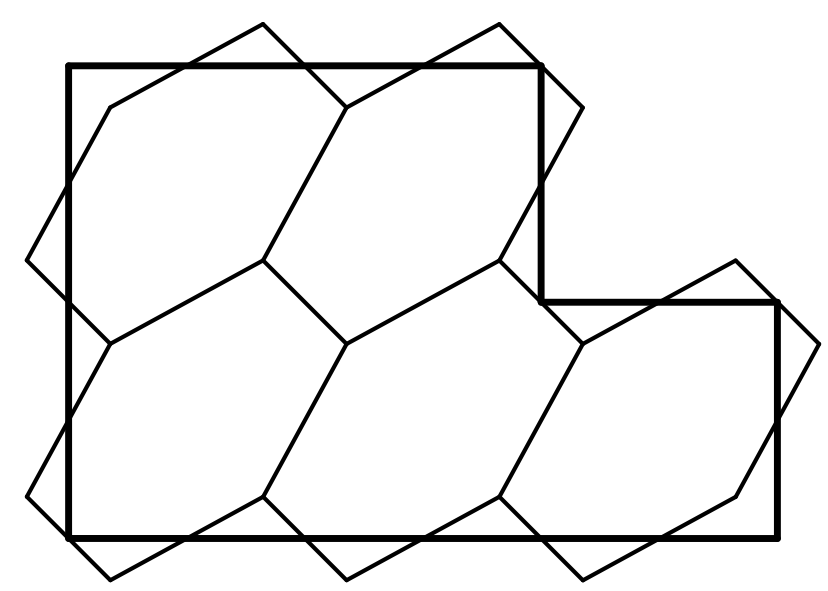

Figura 3.8: Órbita reticulada quadrada definida pela palavra 232313231232323131.

Dado $\hat{Q}_{3}(\omega)$ um caminho fechado, é possível obter de volta $H(\omega)$. $\hat{Q}_{3}(\omega)$ delimita alguns quadrados de $\mathcal{Q}$, e cada um deles tem um hexágono correspondente. $H(\omega)$ é a união de todas as arestas de $\mathcal{H}$ que pertencem um destes hexágonos e interceptam $\hat{Q}_{3}(\omega)$. Em outras palavras, $H(\omega)$ é a união de todas as arestas de $\mathcal{H}$ que interceptam $\hat{Q}_{3}(\omega)$, com exceção de arestas do tipo 3 que estão em vértices "noroeste" ou "sudeste" de $\hat{Q}_{3}(\omega)$.

Dado $\hat{Q}_{3}(\omega)$, também é fácil obter o desdobramento $U(\omega,$.$) de um triângulo qualquer: cada$ aresta de $\hat{Q}_{3}(\omega)$ é um $k$-leque maximal, onde $k$ é o número de quadrados desta aresta (neste caso, a aresta tem comprimento $2 k$ pois cada quadrado do ladrilhamento tem lado 2). Veja a figura 3.8. Além disso, dois leques maximais consecutivos estão no mesmo lado da sua aresta 3 em comum se, e somente se, o vértice de $\hat{Q}_{3}(\omega)$ correspondente a esta aresta não está contido em $H(\omega)$, já que temos o trecho 12 ou 21 em $\omega$.

\subsubsection{Funções comparadoras}

Fixada uma palavra e dado o desdobramento de um triângulo $X=(\alpha, \beta)$ com relação a ela, estamos interessamos em, dados dois pontos $a$ e $b$ neste desdobramento, definir uma função $F[a, b](X)$ que nos permita afirmar se $p$ está acima, abaixo ou na mesma altura que $q$. Note que nesta notação está suprimida a dependência da palavra, pois sempre trabalharemos com uma palavra ou uma família de palavras fixadas.

Isto será útil pois, se rotacionarmos o desdobramento de modo que a translação entre o primeiro e o último lado seja horizontal, a palavra que gerou este desdobramento representa uma órbita bilhar periódica se e somente se todos os vértices superiores estão acima de todos os vértices inferiores, pois neste caso há um segmento ligando o primeiro e o último lado inteiramente contido no desdobramento e não passando por nenhum vértice.

Muitas vezes conseguiremos usar o lema do leque para reduzir o número de vértices a verificar e, assim, se torna fácil checar usando as funções comparadores se existe órbita bilhar periódica. Com tais funções, também é possível algoritmizar este processo de verificação, como é feito com parte dos resultados da seção 2.3 .

Vejamos agora como definiremos as funções comparadoras $F$. Podemos denominar cada aresta de $\mathcal{H}$ pelas coordenadas inteiras de seu ponto médio. Para toda órbita $\Gamma(\omega)$, fixamos a origem da órbita reticulada hexagonal $H(\omega)$ no ponto $P_{0}$. Dada uma aresta $e$ do desdobramento, denotamos por $\hat{e} \in \mathbb{Z}^{2}$ sua representação em coordenadas inteiras. Assim, a aresta $(0,0)$ corresponde a aresta que representa no desdobramento o último dígito de $\omega$. Veja a figura 3.9.

O lema a seguir dá um significado geométrico para estas coordenadas. Nós as usaremos no decorrer deste capítulo para definir uma função que diz se um ponto está acima, abaixo ou na mesma altura de outro no desdobramento. 


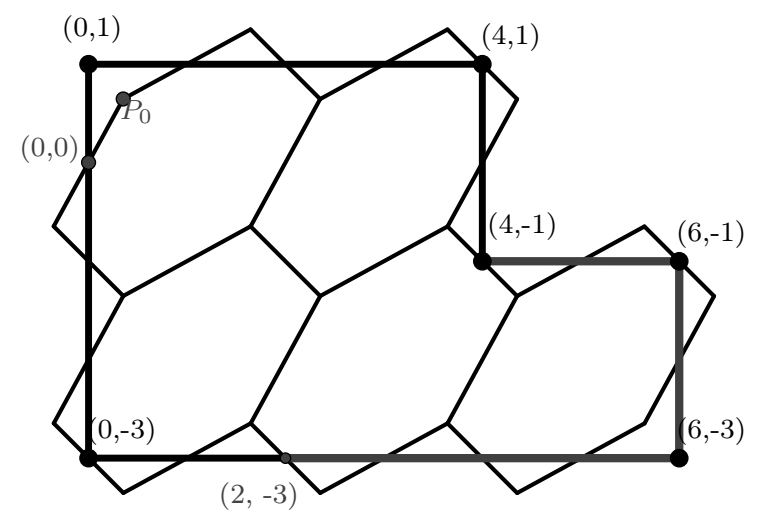

Figura 3.9: As arestas do desdobramento são nomeadas de acordo com sua coordenada na órbita reticulada.

Lema 4. Seja $X=(\alpha, \beta) \in \Delta$ e $e_{1}$ e $e_{2}$ duas arestas do desdobramento $U\left(\omega, T_{X}\right)$. Seja $\theta\left(e_{1}, e_{2}\right) o$ ângulo no sentido anti-horário pelo qual devemos rotacionar $e_{1}$ para que fique paralelo a $e_{2}$. Então $\theta\left(e_{1}, e_{2}\right)=\left\langle X, \hat{e}_{2}-\hat{e}_{1}\right\rangle$.

Demonstração. Faremos o argumento para arestas adjacentes do desdobramento, e ele pode ser estendido para os demais pares de arestas.

Temos seis casos:

(i) $e_{2}=e_{1}+(0,1)$ corresponde à reflexão 13 ou $31 .\left\langle X, \hat{e}_{2}-\hat{e}_{1}\right\rangle=\langle(\alpha, \beta),(0,1)\rangle=\beta$, que é o ângulo entre os lados 1 e 3 ;

(ii) $e_{2}=e_{1}+(0,-1)$, é semelhante ao caso anterior, com $e_{1}$ e $e_{2}$ trocados;

(ii) $e_{2}=e_{1}+(1,0)$ corresponde à reflexão 23 ou $32 .\left\langle X, \hat{e}_{2}-\hat{e}_{1}\right\rangle=\langle(\alpha, \beta),(0,1)\rangle=\alpha$, que é o ângulo entre os lados 2 e 3 ;

(iv) $e_{2}=e_{1}+(-1,0)$, é semelhante ao caso anterior, com $e_{1}$ e $e_{2}$ trocados;

(v) $e_{2}=e_{1}+(1,1)$ corresponde à reflexão 12 ou 21. $\left\langle X, \hat{e}_{2}-\hat{e}_{1}\right\rangle=\langle(\alpha, \beta),(1,1)\rangle=\alpha+\beta$, que é o ângulo complementar ao ângulo entre os lados 1 e 2 ;

(vi) $e_{2}=e_{1}+(-1,-1)$, é semelhante ao caso anterior, com $e_{1}$ e $e_{2}$ trocados.

Vamos definir as funções $F$ com base nas arestas 3, do mesmo modo que fizemos com a órbita reticulada quadrada. Dados dois vértices $a$ e $b$ ligamos por arestas 3 , vamos definir F[a,b](X). Ao lidar com as palavras $B_{n}$ no teorema 3.2.1 trataremos do caso em que os pontos não estão ligados só por arestas do tipo 3 .

Sejam $e_{0}, \ldots, e_{m}$ a lista de arestas na 3-espinha, que é a poligonal formada por arestas do tipo 3 que são os extremos de cada leque maximal e está ressaltada na figura 3.10. Como cada segmento da órbita reticulada quadrada $\hat{Q}_{3}(\omega)$ corresponde a um leque maximal, então os pontos de $\mathbb{Z}^{2}$ que representam $e_{0}, \ldots, e_{m}$ são os vértices de $\hat{Q}_{3}(\omega)$, apontados na figura 3.9.

Dados dois pontos $a$ e $b$ do desdobramento que estão ligados por arestas do tipo 3 e $f_{0}, \ldots, f_{n}$ a lista de arestas ligando $a$ e $b$. Definimos

$$
\begin{gathered}
P[a, b](X):= \pm \sum_{j=0}^{n}(-1)^{j} \exp \left(i\left\langle X, \hat{f}_{j}\right\rangle\right) \\
Q(X):=\sum_{j=0}^{m}(-1)^{j} \exp \left(i\left\langle X, \hat{e}_{j}\right\rangle\right)
\end{gathered}
$$




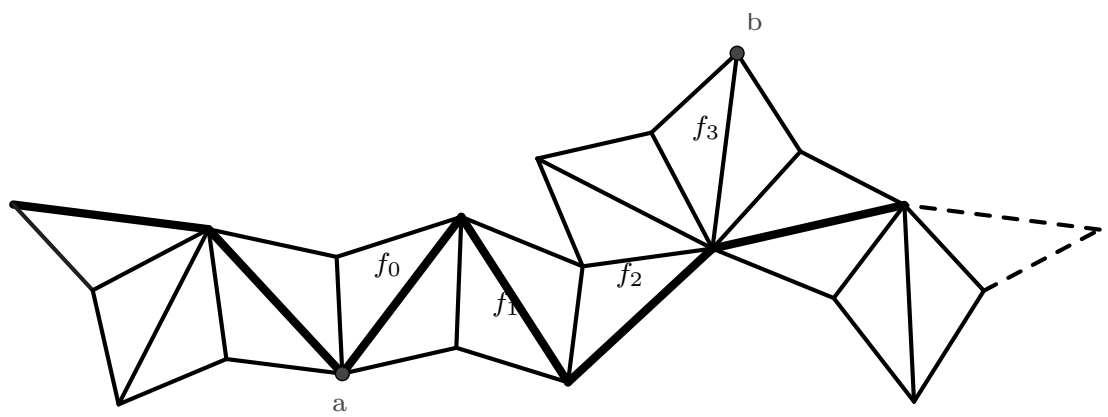

Figura 3.10: Desdobramento da palavra 232313231232323131 com relação ao triângulo $X=\left(35^{\circ}, 40^{\circ}\right)$. Vamos calcular a função comparadora para os pontos a e b.

Onde $\hat{e} \in \mathbb{Z}$ é o ponto que representa a aresta $e$ do desdobramento. Note também que $\hat{Q}$ é a órbita reticulada e $Q$ é a função que corresponde a esta órbita.

O sinal que acompanha $P$ é determinado do seguinte modo: associamos a cada vértice de $\hat{Q}_{3}(\omega)$ um sinal alternado, começando por + . Se o primeiro vértice que representa uma aresta 3 do caminho entre $a$ e $b$ coincidir com um vértice de $\hat{Q}_{3}(\omega)$, mantemos este sinal. Este é o único caso que trataremos por enquanto.

Definição 3.2.1. A função comparadora $F$ para os pontos a e $b$ no desdobramento de uma palavra com relação ao triângulo $X$ é definida por

$$
F[a, b](X):=\Im(P[a, b](X) \overline{Q(X)}),
$$

isto é, a parte imaginária do produto entre $P[a, b](X)$ e o complexo conjugado de $Q(X)$

Proposição 3.2.1. Dado um ponto a num desdobramento, denotamos por $y_{a}$ sua segunda coordenada cartesiana. Temos:

- $F[a, b](X)>0$ se, e somente se, $y_{a}<y_{b}$;

- $F[a, b](X)=0$ se, e somente se, $y_{a}=y_{b}$;

- $F[a, b](X)<0$ se, e somente se, $y_{a}>y_{b}$.

Demonstração. Pelo lema $4,\langle X, \hat{e}\rangle$ é o ângulo no sentido anti-horário pelo qual devemos rotacionar $(0,0)$ para que fique paralelo a $e$, e $(0,0)$ é o último lado do desdobramento.

Logo, se fixamos o primeiro lado do desdobramento horizontalmente, $Q(X)$ é um número complexo cujo vetor que o representa tem mesmo ângulo que a inclinação da translação que leva o primeiro ao último lado do desdobramento. Portanto, $\overline{Q(X)}$ é um número complexo que, se for multiplicado em todos os pontos do desdobramento, rotaciona o desdobramento de modo que a translação entre o primeiro e o último lado é horizontal (além de mudar a proporção do desdobramento de acordo com o comprimento $l>0$ desta translação).

Se, com o primeiro lado horizontal, fixamos o comprimento do lado 3 como 1 , então $Q(X)$ é o vetor que leva o primeiro lado ao último.

Do mesmo modo, $P[a, b](X)$ é o vetor que aponta do vértice $a$ para o vértice $b$ se o primeiro lado for fixado horizontalmente. Logo, $P[a, b](X) \overline{Q(X)}$ tem mesma inclinação que o vetor que aponta do vértice $a$ para o vértice $b$ no desdobramento rotacionado para que a translação entre o primeiro e o último lado seja horizontal. Mais precisamente, $P[a, b](X) \overline{Q(X)}$ é este vetor que aponta de $a$ para $b$ multiplicado por $l$. Portanto, $\Im(P[a, b](X) \overline{Q(X)})=l\left(y_{b}-y_{a}\right)$.

Dada uma palavra $\omega, a_{i}$ seus vértices superiores e $b_{i}$ os inferiores, então o ladrilho de órbita $O(\omega)$ é definido como a região de $\Delta$ tal que $F\left[a_{i}, b_{j}\right](X)<0$ quando $a_{i}$ está à esquerda de $b_{j}$ e $F\left[b_{j}, a_{i}\right](X)>0$ quando $a_{i}$ está à direita de $b_{j}$. A fronteira de $O(W)$ é a união dos $X \in \Delta$ tais que $F\left[a_{i}, b_{j}\right](X)=0$ para todo $i$ e $j$. 
Vejamos um exemplo de calculo de $F[a, b](X) \operatorname{com} \omega=232313231232323131, X=\left(35^{\circ}, 40^{\circ}\right)=$ $\left(\frac{7 \pi}{36}, \frac{2 \pi}{9}\right)$ e $a$ e $b$ indicados na figura 3.10 .

Temos a seguinte lista de vértices de $\hat{Q}_{3}(\omega): \hat{e}_{0}=(0,1), \hat{e}_{1}=(4,1), \hat{e}_{2}=(4,-1), \hat{e}_{3}=(6,-1)$, $\hat{e}_{4}=(6,-3)$ e $\hat{e}_{5}=(0,-3)$. Os vértices que correspondem às arestas 3 ligando $a$ e $b$ são: $\hat{f}_{0}=(4,-1)$, $\hat{f}_{1}=(6,-1), \hat{f}_{2}=(6,-3), \hat{f}_{3}=(2,-3)$. Temos então

$$
\begin{aligned}
P[a, b]\left(\frac{7 \pi}{36}, \frac{2 \pi}{9}\right) & =\sum_{j=0}^{3}(-1)^{j} \exp \left(i\left\langle\left(\frac{7 \pi}{36}, \frac{2 \pi}{9}\right), \hat{f}_{j}\right\rangle\right) \\
& =\exp \left(i \frac{5 \pi}{9}\right)-\exp \left(i \frac{17 \pi}{18}\right)+\exp \left(i \frac{\pi}{2}\right)-\exp \left(-i \frac{5 \pi}{18}\right) \approx 2,58 \exp \left(i \times 86,26^{\circ}\right), \\
Q\left(\frac{7 \pi}{36}, \frac{2 \pi}{9}\right) & =\sum_{j=0}^{5}(-1)^{j} \exp \left(i\left\langle\left(\frac{7 \pi}{36}, \frac{2 \pi}{9}\right), \hat{e}_{j}\right\rangle\right) \\
& =\exp \left(i \frac{2 \pi}{9}\right)-\exp (i \pi)+\exp \left(i \frac{5 \pi}{9}\right)-\exp \left(i \frac{17 \pi}{18}\right)+\exp \left(i \frac{\pi}{2}\right)-\exp \left(-i \frac{2 \pi}{3}\right) \\
\hline\left(\frac{7 \pi}{36}, \frac{2 \pi}{9}\right) & =\exp \left(-i \frac{2 \pi}{9}\right)+1+\exp \left(-i \frac{5 \pi}{9}\right)-\exp \left(-i \frac{17 \pi}{18}\right)-i-\exp \left(i \frac{2 \pi}{3}\right) \\
& \approx 4,53 \exp \left(i \times 47,17^{\circ}\right)
\end{aligned}
$$

e

$$
F[a, b]\left(\frac{7 \pi}{36}, \frac{2 \pi}{9}\right) \approx \Im\left(11,69 \exp \left(i \times 39.09^{\circ}\right)\right)>0 .
$$

Logo $y_{a}<y_{b}$. O significado geométrico de $P$ e $Q$ pode ser visto na figura 3.11.

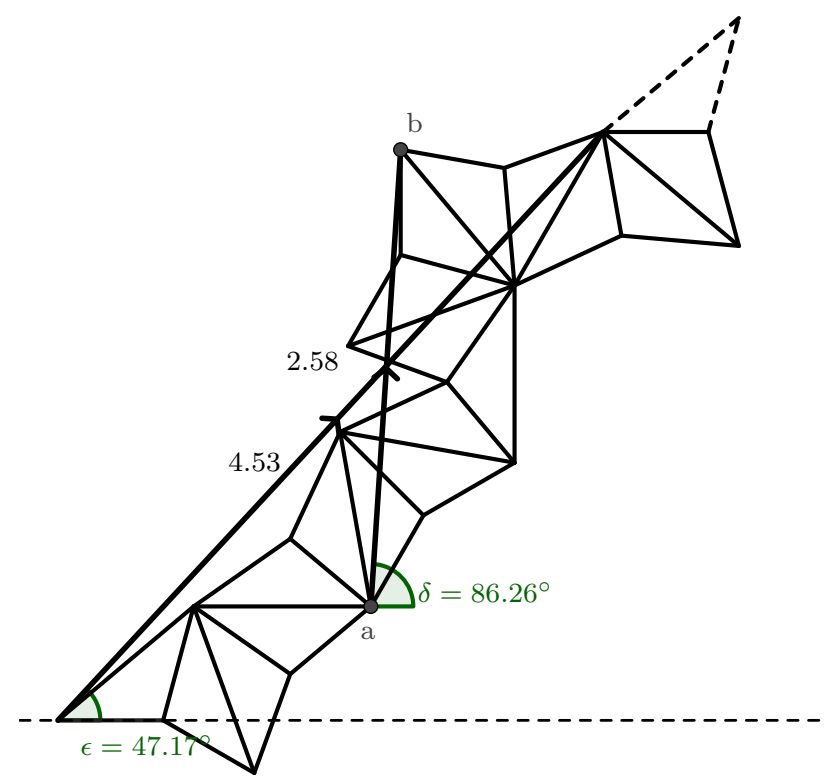

Figura 3.11: Desdobramento da palavra 232313231232323131 com relação ao triângulo $X=\left(35^{\circ}, 40^{\circ}\right)$ com primeiro lado horizontal. Se o lado 3 tem comprimento $1, P[a, b](X)$ é o número complexo cujo vetor que o representa aponta de a para $b$ e $Q(X)$ é o número complexo cujo vetor que o representa leva o primeiro lado ao último. 


\subsubsection{Teorema 3.2 .1}

Seja $B(X, \varepsilon)$ a bola de centro $X$ e raio $\varepsilon$. Defino $B_{ \pm \pm}(X, \varepsilon)$ como a interseção de $B(X, \varepsilon)$ com o quadrante $( \pm, \pm)$ aberto. Aqui, definimos cada quadrante considerando os quadrantes do plano cartesiano caso a origem fosse no ponto $X$.

Teorema 3.2.1. Para todo $n>3$ existem palavras $A_{n}, B_{n}$ e $C_{n}$ e um $\varepsilon_{n}>0$ tais que

(i) $B_{--}\left(V_{n}, \varepsilon_{n}\right) \subset O\left(A_{n}\right)$

(ii) $B_{-+}\left(V_{n}, \varepsilon_{n}\right) \subset O\left(B_{n}\right)$

(ii) $B_{+-}\left(V_{n}, \varepsilon_{n}\right) \subset O\left(C_{n}\right)$

(iv) $B\left(V_{n}, \varepsilon_{n}\right) \backslash \overline{B_{++}\left(V_{n}, \varepsilon_{n}\right)} \subset O\left(A_{n}\right) \cup O\left(B_{n}\right) \cup O\left(C_{n}\right)$

O ultimo item do teorema 3.2.1 cobre os segmentos da fronteira de $B_{--}\left(V_{n}, \varepsilon_{n}\right)$.

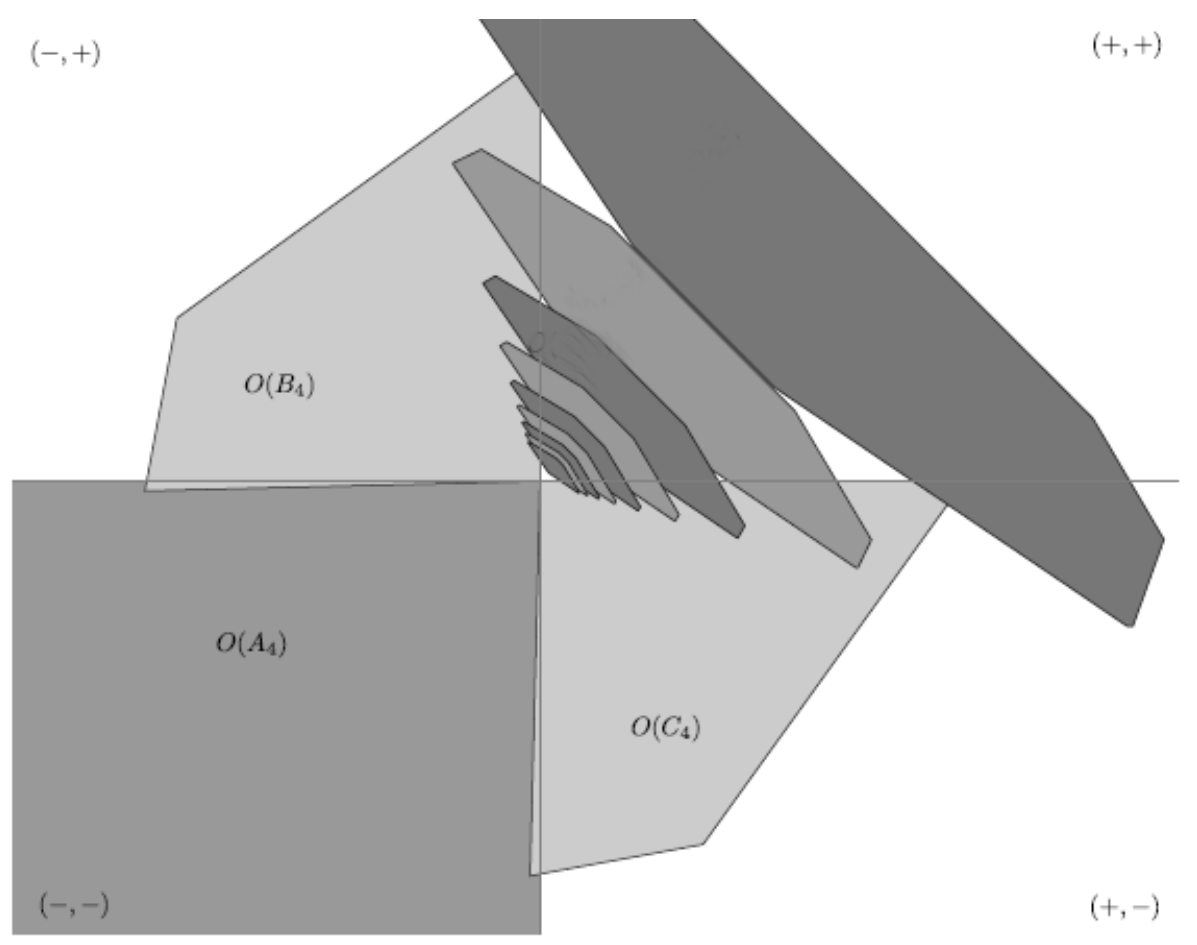

Figura 3.12: Ilustração dos ladrilhos de órbita do teorema 3.2.1 e 3.3.1 na vizinhança do ponto $V_{8}$. Esta figura foi gerada pelos autores de [HS09].

Demonstração. Em cada item a seguir, mostraremos que os "quartos de bola" $B_{ \pm \pm}\left(V_{n}, \varepsilon\right)$ estão no ladrilho de órbita correspondente para algum $\varepsilon$. Tomamos $\varepsilon_{n}$ como o menor deles.

(i) A família de palavras que usaremos neste caso é $A_{n}=\left(1(31)^{n-1}(32)^{n}\right)^{2}$. No primeiro caso do teorema 3.2.1, mostramos que todo triângulo isósceles de ângulos agudos $\alpha \in\left[\frac{\pi}{2 n+1}, \frac{\pi}{2 n}\right)$ pertence a $O\left(A_{n}\right)$. Sabemos que $A_{n}$ é estável, mas não sabemos qual é a vizinhança de $\Delta$ onde esta órbita existe. Vejamos agora que existe $\varepsilon$ tal que todo $T_{X} \operatorname{com} X \in B_{--}\left(V_{n}, \varepsilon_{n}\right)$ (não necessariamente isósceles) pertence a $O\left(A_{n}\right)$.

$U\left(A_{n},.\right)$ é a união de quatro $n$-leques maximais. Como $V_{n}=\left(\frac{\pi}{2 n}, \frac{\pi}{2 n}\right)$, em $U\left(A_{n}, V_{n}\right)$ cada leque tem ângulo $\pi$. Portanto, existe uma linha horizontal $l$ passando pela base de cada leque. Veja a figura 3.13 . 


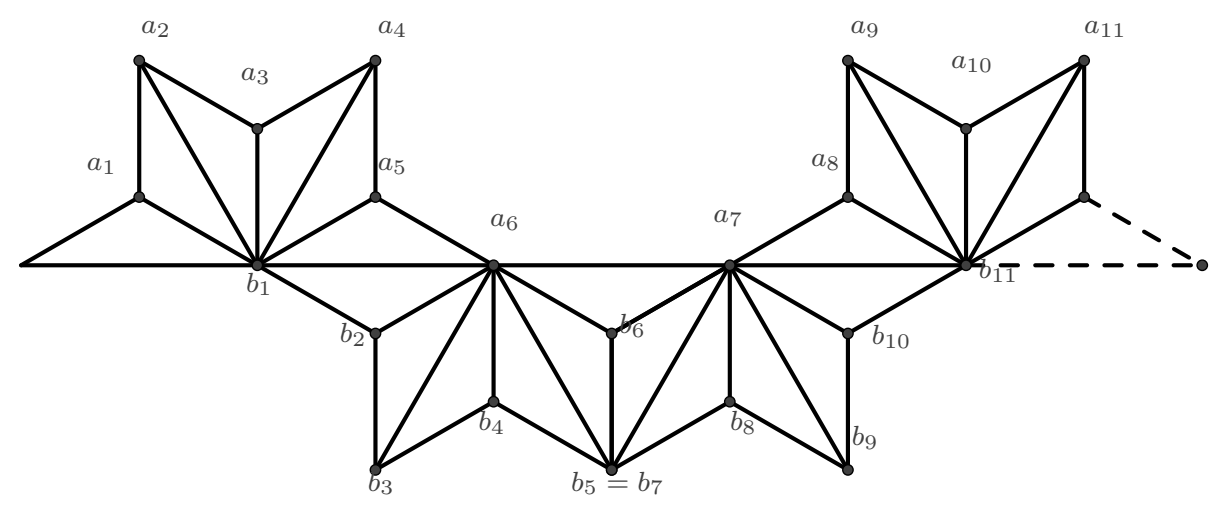

Figura 3.13: Desdobramento da palavra $A_{3}=1313132323213131323232$ no triângulo $V_{3}$.

Sejam $a_{1}, \ldots, a_{4 n-1}$ os vértices superiores e $b_{1}, \ldots, b_{4 n-1}$ os vértices inferiores do desdobramento $U\left(A_{n}, V_{n}\right)$. Então $b_{1}, a_{2 n}, a_{2 n+1}$ e $b_{4 n-1}$ pertencem a $l$.

Queremos mostrar que existe $\varepsilon>0$ tal que para todo $X \in B_{--}\left(V_{n}, \varepsilon\right)$, o desdobramento $U\left(A_{n}, X\right)$ contém uma órbita periódica. Como vamos diminuir os ângulos agudos, para $\varepsilon$ pequeno, os leques que apontam pra cima continuam apontando pra cima e os que apontam pra baixo continuam apontando pra baixo. Além disso, o ângulo entre o primeiro lado e a linha central é menor que $\frac{\pi}{2}$, logo o vértice superior mais baixo e o vértice inferior mais alto continuam entre os vértices $b_{1}, a_{2 n}, a_{2 n+1}$ e $b_{4 n-1}$. Logo, pelo lema 2 , basta mostrar que $a_{2 n}$ e $a_{2 n+1}$ estão acima de $b_{1}$ e $b_{4 n-1}$.

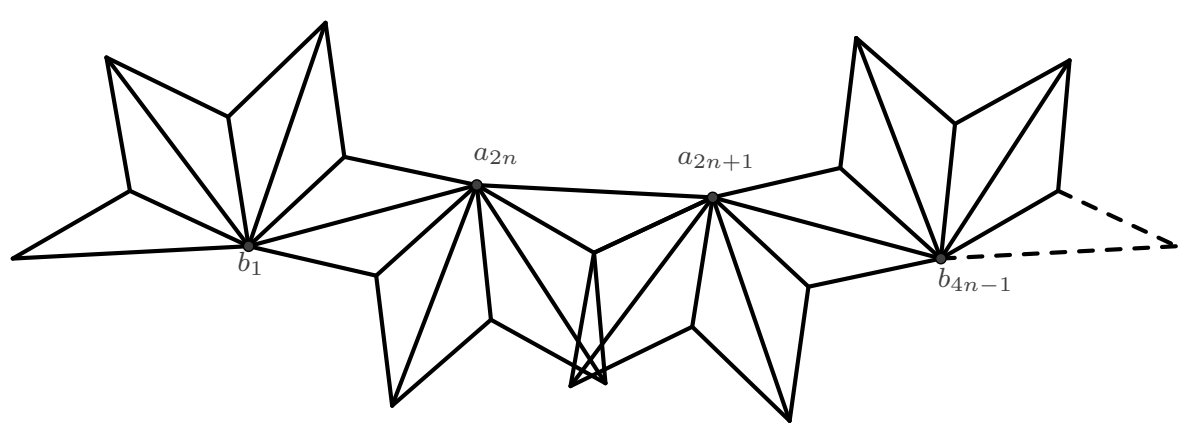

Figura 3.14: Desdobramento da palavra $A_{3}=1313132323213131323232$ em um triângulo $(\alpha, \beta)$ com $\alpha, \beta<$ $\frac{\pi}{6}$.

Poderíamos calcular as posições relativas de $a_{2 n}, a_{2 n+1}, b_{1}$ e $b_{4 n-1}$ com base em seus ângulos como fizemos no teorema 3.1.1. Em vez disso, vamos usar as funções comparadoras.

Primeiramente, note que que a órbita reticulada quadrada de $A_{n}$ é um quadrado de vértices $\hat{e}_{0}=(-1,0), \hat{e}_{1}=(-1,-2 n), \hat{e}_{2}=(2 n-1,-2 n)$ e $\hat{e}_{3}=(2 n-1,0)$. Temos

$$
P\left[b_{1}, a_{2 n}\right](\alpha, \beta)=-\exp (i(-\alpha-2 n \beta))
$$

e

$$
Q(\alpha, \beta)=\exp (-i \alpha)-\exp (i(-\alpha-2 n \beta))+\exp (i((2 n-1) \alpha-2 n \beta))-\exp (i(2 n-1) \alpha),
$$

$\operatorname{logo}, F\left[b_{1}, a_{2 n}\right](\alpha, \beta)=\sin (2 n \beta)+\sin (2 n \alpha)-\sin (2 n(\alpha+\beta))$, que é positivo para $\alpha<\frac{\pi}{2 n}$ e $\beta<\frac{\pi}{2 n}$ próximos de $\frac{\pi}{2 n}$, pois cada parcela é positiva. 
Do mesmo modo, temos que $F\left[a_{2 n+1}, b_{4 n-1}\right](\alpha, \beta)=-\sin (2 n \beta)-\sin (2 n \alpha)+\sin (2 n(\alpha+\beta))<0$.

Por fim, temos

$$
\begin{aligned}
F\left[b_{1}, a_{2 n+1}\right](\alpha, \beta) & =2 \sin (2 n \beta)-\sin (2 n(\alpha+\beta))+\sin (2 n(\alpha-\beta)) \\
& =8 \sin ^{2}(n \alpha) \sin (n \beta) \cos (n \beta)>0 \text { para } \beta \in\left(0, \frac{\pi}{2 n}\right)
\end{aligned}
$$

e

$$
\begin{aligned}
F\left[a_{2 n}, b_{4 n-1}\right](\alpha, \beta) & =\sin (2 n(\alpha-\beta))+\sin (2 n(\alpha+\beta))-2 \sin (2 n \alpha) \\
& =-8 \sin ^{2}(n \beta) \sin (n \alpha) \cos (n \alpha)<0 \text { para } \alpha \in\left(0, \frac{\pi}{2 n}\right) .
\end{aligned}
$$

(ii, iii e iv) Tratemos dos demais itens agora. Mostraremos que pra todo $n \geq 4$ existe $\varepsilon>0$ tal que $B_{-+}\left(V_{n}, \varepsilon\right) \subset O\left(B_{n}\right)$. Trocando os 1's por 2's em $B_{n}$, obtemos $C_{n}$, que terá as mesmas propriedades de $B_{n}$ no ladrilho simétrico com relação a reta $\alpha=\beta$. Além disso vamos ver que o segmento $\overline{B_{--}\left(V_{n}, \varepsilon\right)} \cap \overline{B_{-+}\left(V_{n}, \varepsilon\right)} \backslash\left\{V_{n}\right\}$ está contido em $B_{n}$. Por simetria, o outro segmento da fronteira de $B_{--}\left(V_{n}, \varepsilon\right)$, que é $\overline{B_{--}\left(V_{n}, \varepsilon\right)} \cap \overline{B_{+-}\left(V_{n}, \varepsilon\right)} \backslash\left\{V_{n}\right\}$, estará contido em $C_{n}$.

Vamos definir $B_{n}$ indutivamente. A primeira palavra da família $B_{n}$ é

$$
\begin{array}{r}
B_{4}=23232313131232323231313132323213131313232323121223 \\
13232313131323232321313132323132313132323231313131,
\end{array}
$$

que tem comprimento 100. O desdobramento desta palavra no triângulo $V_{4}$ pode ser visto na figura 3.15. A órbita reticulada quadrada de $B_{4}$ é a figura 3.16 .

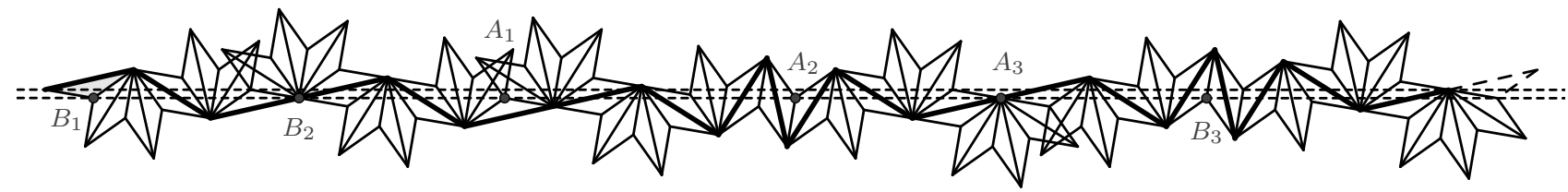

Figura 3.15: Desdobramento da palavra $B_{4}$ no triângulo $V_{4}$, rotacionada de modo que a translação do primeiro para o último lado é horizontal.

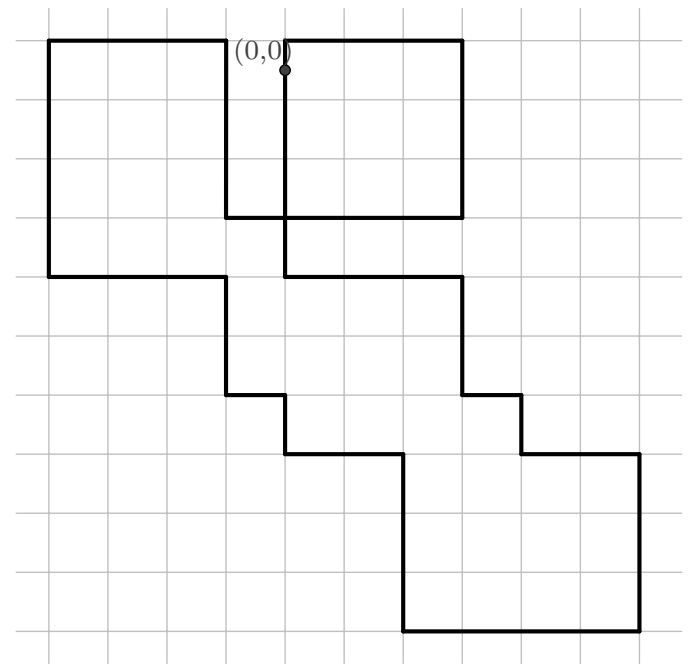

Figura 3.16: $\hat{Q}_{3}\left(B_{4}\right)$.

Dada $B_{n}$, obtemos $B_{n+1}$ aumentando em 2 (que corresponde a um quadrado do ladrilhamento $\mathcal{Q}$ do plano) cada um de seus lados. A figura 3.17 mostra a órbita reticulada quadrada de $B_{5}$. 


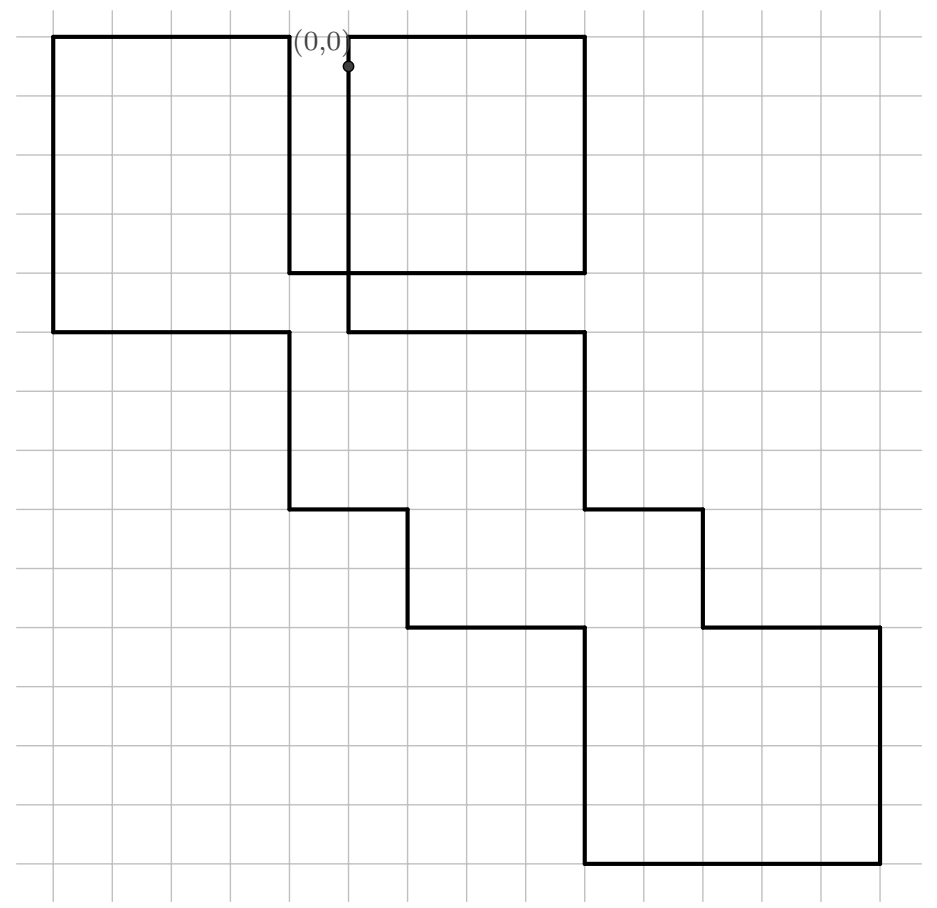

Figura 3.17: $\hat{Q}_{3}\left(B_{5}\right)$.

De maneira geral, temos que os 20 vértices de $\hat{Q}_{3}\left(B_{n}\right)$, na ordem em que são percorridos, são $\hat{e}_{0}=(0,1), \hat{e}_{1}=(2 n-2,1), \hat{e}_{2}=(2 n-2,-2 n+3), \hat{e}_{3}=(-2,-2 n+3), \hat{e}_{4}=(-2,1), \hat{e}_{5}=(-2 n, 1)$, $\hat{e}_{6}=(-2 n,-2 n+1), \hat{e}_{7}=(-2,-2 n+1), \hat{e}_{8}=(-2,-4 n+5), \hat{e}_{9}=(2 n-8,-4 n+5), \hat{e}_{10}=$ $(2 n-8,-6 n+11), \hat{e}_{11}=(4 n-12,-6 n+11), \hat{e}_{12}=(4 n-12,-8 n+13), \hat{e}_{13}=(6 n-12,-8 n+13)$, $\hat{e}_{14}=(6 n-12,-6 n+11), \hat{e}_{15}=(4 n-8,-6 n+11), \hat{e}_{16}=(4 n-8,-4 n+5), \hat{e}_{17}=(2 n-2,-4 n+5)$, $\hat{e}_{18}=(2 n-2,-2 n+1)$ e $\hat{e}_{19}=(0,-2 n+1)$.

Queremos calcular $Q\left(V_{n}\right)$ pois a usaremos para calcular as funções comparadora de alguns pontos chave durante esta demonstração no triângulo $V_{n}$ e, utilizando derivada, mostraremos que $B_{n}$ é uma órbita bilhar periódica em $B_{-+}\left(V_{n}, \varepsilon\right)$.

$$
\begin{aligned}
Q\left(V_{n}\right) & =\sum_{j=0}^{19}(-1)^{j} \exp \left(i \frac{\pi}{2 n}\left(\hat{e}_{j, 1}+\hat{e}_{j, 2}\right)\right) \\
& =\sum_{j=0}^{19}(-1)^{j} \exp \left(i \frac{\pi}{2 n}\right)^{\left(\hat{e}_{j, 1}+\hat{e}_{j, 2}\right)}
\end{aligned}
$$

Seja $w_{n}=\exp \left(i \frac{\pi}{2 n}\right) \mathrm{e}$

$$
L(j)= \begin{cases}\hat{e}_{j, 1}+\hat{e}_{j, 2} \quad \bmod 4 n & \text { se } j \text { é par; } \\ \hat{e}_{j, 1}+\hat{e}_{j, 2}+2 n \quad \bmod 4 n & \text { se } j \text { é ímpar. }\end{cases}
$$

Então, $\operatorname{como} \exp (i \pi)=-1$,

$$
\begin{aligned}
Q\left(V_{n}\right) & =\sum_{j=0}^{19} w_{n}^{L(j)} \\
& =8 w_{n}+4 w_{n}^{3}+6 w_{n}^{-1}+2 w_{n}^{-3} .
\end{aligned}
$$

O maior leque maximal que aparece em $B_{n}$ é um $n$-leque. Como todo ponto $X=(\alpha, \beta)$ sufi- 
cientemente próximo de $V_{n}$ satisfaz $\max (\alpha, \beta) \leq \frac{\pi}{\delta\left(B_{n}\right)}=\frac{\pi}{n}$ com relação à palavra $B_{n}$, pelo lema 2 , temos que mostrar que todo vértice base superior de $U\left(B_{n}, T_{X}\right)$ esta acima de cada vértice base inferior, assim concluímos que existe órbita bilhar periódica.

Para $X=V_{n}$, temos 6 vértices base na mesma altura que são os 3 superiores mais baixos e os 3 inferiores mais altos: $A_{1}, A_{2}, A_{3}, B_{1}, B_{2}$ e $B_{3}$. Veja a figura 3.15. Note que, mesmo ilustrando os vértices no desdobramento $U\left(B_{4}, V_{4}\right)$, pela definição de $B_{n}$, o número de leques não muda, apenas a quantidade de triângulos em cada um deles. Temos sempre 20 leques maximais. Usando a notação de [HS09], podemos denominar os vértices base pelo número $k$ do leque onde ele está, se ele está à esquerda $(\mathrm{L})$ ou à direita $(\mathrm{R})$ do centro e se ele é interior $(\mathrm{I})$ ou exterior $(\mathrm{O})$. Assim, o vértice base do segundo leque que está à esquerda e é mais externo é denotado por $(2, L, O)$. Note que esta notação pode repetir vértices, por exemplo, $(1, R, I)=(2, L, O)$. Assim, temos para todo $n$ que $A_{1}=(5, R, I), A_{2}=(10, R, I), A_{3}=(12, R, O), B_{1}=(1, L, I), B_{2}=(4, L, O)$ e $B_{3}=(16, L, I)$.

De fato, vamos usar $Q\left(V_{n}\right)$ para calcular as funções comparadoras necessárias para afirmar que os vértices de interesse acima listados estão na mesma altura. Mais precisamente, dado um vértice base superior $a$ diferente de $A_{1}, A_{2}$ e $A_{3}$, mostraremos que $a$ está acima de $B_{1}$, mostrando que $F\left[B_{1}, a\right]\left(V_{n}\right)>0$. Analogamente, dado um vértice base inferior $b$ diferente de $B_{1}, B_{2}$ e $B_{3}$, mostraremos que $b$ está abaixo de $B_{1}$, mostrando que $F\left[B_{1}, b\right]\left(V_{n}\right)<0$. Por fim, se $c$ for um vértice base entre $A_{1}, A_{2}, A_{3}, B_{2}$ e $B_{3}$, mostraremos que $F\left[B_{1}, c\right]\left(V_{n}\right)=0$, ou seja, estão todos na mesma altura.

As funções comparadoras que usaremos a seguir são uma adaptação da definição 3.2.1, uma vez que $B_{1}$ não está ligado a uma aresta do tipo 3. Ainda assim, vamos usar a 3-espinha o máximo possível. Dado um vértice $p$ do desdobramento, ligamos $B_{1}$ a $a_{1}=(1, L, O)$ pelo lado 1 do primeiro triângulo, percorremos a 3-espinha até o ponto $p^{\prime}$ mais próximo antes de $p$ usando as arestas $e_{0}, \ldots, e_{s}$, e depois podemos seguir por uma aresta $3(\delta=0)$, ir para cima $(\delta=1)$ ou para baixo $(\delta=-1)$. Veja a figura 3.18 .

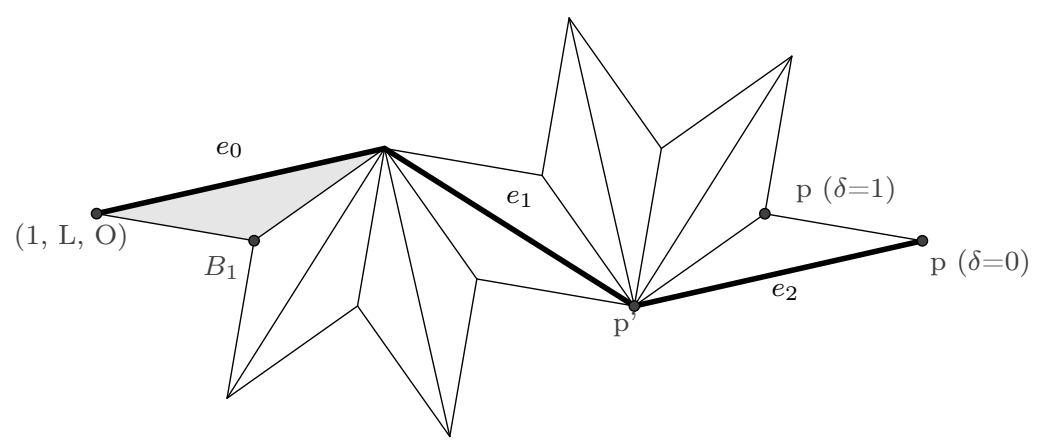

Figura 3.18: Para calcular $F$ de dois pontos que não estão ligados apenas por arestas do tipo 3, uso estas arestas para me aproximar dos pontos e depois adiciono um fator referente ao caminho que falta percorrer.

Ainda considerando que os lados 3 tem comprimento 1 , temos que os lados menores de $V_{n}$ tem comprimento $\lambda=\frac{1}{2 \cos (\pi / 2)}=\frac{1}{w_{n}+w_{n}^{-1}}$. Assim, dado um vértice $p$ do desdobramento que está imediatamente ligado a $e_{s}$ por uma aresta do tipo $\delta$, como descrito acima, temos que

$$
P(s, \delta):=P\left[B_{1}, p\right]\left(V_{n}\right)=-\lambda+\sum_{j=0}^{s} w_{n}^{L(j)}+\lambda^{|\delta|} w_{n}^{L(s+1)+\delta} .
$$

Hooper e Schwartz usam esta expressão para mostrar que $A_{1}, A_{2}, A_{3}, B_{2}$ e $B_{3}$ estão na mesma altura que $B_{1}$, os demais vértices base superiores estão acima e os demais vértices base inferiores estão abaixo. Uma tabela com o cálculo de $P(s, \delta)$ para $s \in\{0, \ldots, 19\}$ e $\delta \in\{-1,01\}$ pode ser consultada na página 32 de [HS09]. Vejamos aqui o cálculo de $P(1,0)$ e $P(1,1)$ que correspondem a $B_{2}=(4, L, O)$ e $(3, L, I)$, respectivamente. 


$$
\begin{gathered}
P(1,0)=-\lambda+w_{n}+w_{n}^{-1}+w_{n}=\frac{2 w_{n}^{2}+2 w_{n}^{4}+1}{w_{n}\left(w_{n}^{2}+1\right)} \\
\Longrightarrow P(1,0) \overline{Q\left(V_{n}\right)}=4 w_{n}^{-4}+12 w_{n}^{-2}+18+12 w_{n}^{2}+4 w_{n}^{4} \\
\Longrightarrow F\left[B_{1}, B_{2}\right]\left(V_{n}\right)=\Im\left(P(1,0) \overline{Q\left(V_{n}\right)}\right)=0 \\
P(1,1)=-\lambda+w_{n}+w_{n}^{-1}+\lambda w_{n}^{2}=\frac{w_{n}^{2}+2 w_{n}^{4}+1}{w_{n}\left(w_{n}^{2}+1\right)} \\
\Longrightarrow P(1,1) \overline{Q\left(V_{n}\right)}=4 w_{n}^{-4}+8 w_{n}^{-2}+14+10 w_{n}^{2}+4 w_{n}^{4} \\
\Longrightarrow F\left[B_{1},(3, L, I)\right]\left(V_{n}\right)=\Im\left(P(1,1) \overline{Q\left(V_{n}\right)}\right)=\Im\left(2 w_{n}^{2}\right)=2 \sin \left(\frac{\pi}{n}\right)>0 .
\end{gathered}
$$

Agora que verificamos que os 6 vértices $A_{1}, A_{2}, A_{3}, B_{1}, B_{2}$ e $B_{3}$ são os que determinam, pelo lema 2, se em um triângulo $X$ próximo de $V_{n}$ a palavra $B_{n}$ representa uma órbita bilhar periódica, vamos mostrar que $A_{1}, A_{2}$ e $A_{3}$ estão acima de $B_{1}, B_{2}$ e $B_{3}$ para $X \in B_{-+}\left(V_{n}, \varepsilon\right)$.

Para isso, vamos mostrar que $H_{i, j}(X):=y_{A_{i}}-y_{B_{i}}>0$ para todo $(i, j) \in\{1,2,3\}^{2}$. Sabemos que $H_{i, j}\left(V_{n}\right)=0$ para todo $i, j$, então vejamos que $\frac{\partial H_{i, j}}{\partial \alpha}\left(V_{n}\right)<0$ e $\frac{\partial H_{i, j}}{\partial \beta}\left(V_{n}\right) \geq 0$, com a igualdade valendo na última inequação se e somente se $(i, j)=(3,2)$. De fato, isto implica que o valor de $H_{i, j}(\alpha, \beta)$ decresce se $\alpha$ cresce e cresce com $\beta$. Logo, como $H_{i j}\left(V_{n}\right)=0, H_{i j}(X)$ será positivo ao diminuir $\alpha$ e aumentar $\beta$ localmente, que é exatamente $X \in B_{-+}\left(V_{n}, \varepsilon\right)$. Além disso, o fato de $\frac{\partial H_{3,2}}{\partial \beta}\left(V_{n}\right)=0$ implica que o segmento $\overline{B_{++}\left(V_{n}, \varepsilon\right)} \cap \overline{B_{-+}\left(V_{n}, \varepsilon\right)} \backslash\left\{V_{n}\right\}$ não está contido em $O\left(B_{n}\right)$, pois nele os vértices base $A_{3}$ e $B_{2}$ estão na mesma altura no desdobramento.

Vamos tratar primeiramente do caso $(i, j)=(3,2)$, mostrando que $H_{3,2}\left(\frac{\pi}{2}, \beta\right)=0$ para todo $\beta$. Para mostrar que $H_{3,2}\left(\frac{\pi}{2}, \beta\right)=0$, basta mostrar que $F\left[B_{2}, A_{3}\right]\left(\frac{\pi}{2}, \beta\right)=0$. Para isso, é necessário calcular $P\left[B_{2}, A_{3}\right]\left(\frac{\pi}{2}, \beta\right)$ e $Q\left(\frac{\pi}{2}, \beta\right)$. A parte da órbita reticular quadrada referente a $\hat{P}$ está tracejada na figura 3.19 .

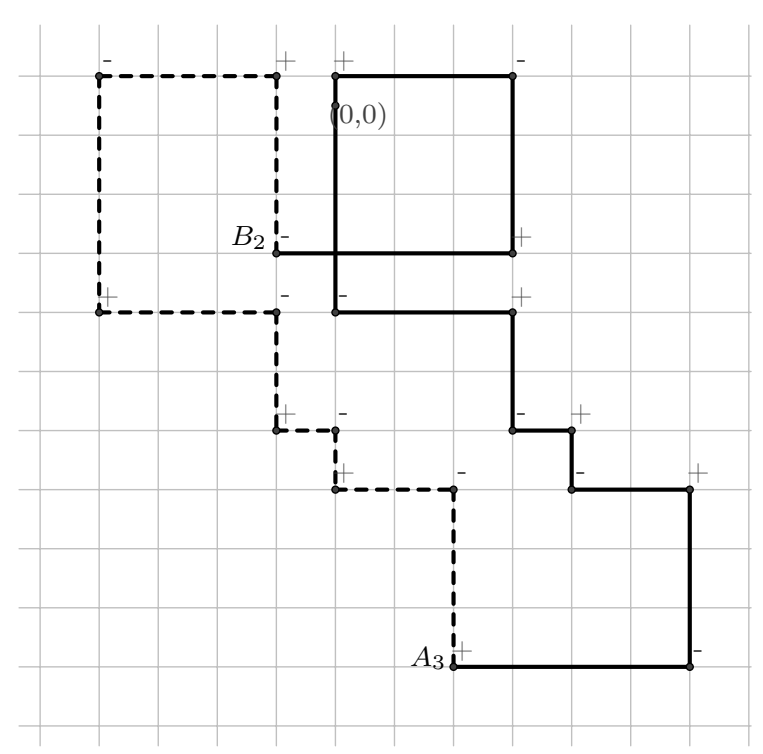

Figura 3.19: $\hat{Q}_{3}\left(B_{4}\right)$. A linha tracejada representa o caminho $\hat{P}$ de $B_{2}$ até $A_{3}$. Os sinais acompanhado cada vértice são referência para o cálculo de $P\left[B_{2}, A_{3}\right](X)$.

Note que os vértices de $\hat{Q}_{3}$ que não estão em $\hat{P}$ são uma translação dos vértices de $\hat{P}$. De fato, a 
translação é dada por $(x, y) \rightarrow(x+2 n, y)$ e o sinal do vértice (usado no calculo de $P$ e $Q)$ é trocado. Então $Q=P\left[B_{2}, A_{3}\right]+P\left[A_{3}, B_{2}\right]$, os dois percorridos no mesmo sentido, então não passam pelos menos vértices. Os vértices usados no calculo de $P\left[A_{3}, B_{2}\right]$ são a translação dos vértices usados no calculo de $P\left[B_{2}, A_{3}\right]$.

Analisemos o que acontece com cada parcela de $P\left[B_{2}, A_{3}\right]$ e $P\left[A_{3}, B_{2}\right]$ em $X=\left(\frac{\pi}{2}, \beta\right)$. Uma parcela da forma $\exp (i(x \alpha+y \beta))$ é sempre levada pela translação para uma da forma $-\exp \left(i\left(\left(x_{2} n\right) \alpha+y \beta\right)\right)$. Quando $\alpha=\frac{\pi}{2},-\exp (i((x+2 n) \alpha+y \beta))=\exp (i(x \alpha+y \beta)), \operatorname{logo}$ $Q\left(\frac{\pi}{2}, \beta\right)=2 P\left[B_{2}, A_{3}\right]\left(\frac{\pi}{2}, \beta\right)$ e temos

$$
F\left[B_{2}, A_{3}\right]\left(\frac{\pi}{2}, \beta\right)=\Im\left(P\left[B_{2}, A_{3}\right]\left(\frac{\pi}{2}, \beta\right) \overline{2 P\left[B_{2}, A_{3}\right]\left(\frac{\pi}{2}, \beta\right)}\right)=0,
$$

pois $P \bar{P}$ é real.

No caso $(i, j) \neq(3,2)$, queremos calcular $\frac{\partial H_{i, j}}{\partial \alpha}\left(V_{n}\right)$ e $\frac{\partial H_{i, j}}{\partial \beta}\left(V_{n}\right)$. Para isso, vamos reescrever $H_{i, j}$ como $F\left[a_{1}, A_{i}\right]-F\left[a_{1}, B_{j}\right]$, lembrando que $a_{1}=(1, L, O)$, e calcular as derivadas destas $F$. Para $A_{3}$ e $B_{2}$ que estão na 3 -espinha, podemos usar o cálculo de $P$ definido na equação 3.1. Temos

$$
\begin{gathered}
P\left[a_{1}, A_{3}\right]\left(V_{n}\right)=\sum_{j=0}^{12}(-1)^{j} \exp \left(i\left\langle X, \hat{e}_{j}\right\rangle\right)=\sum_{j=0}^{12} w_{n}^{L(j)} \\
\text { e } P\left[a_{1}, B_{2}\right]\left(V_{n}\right)=\sum_{j=0}^{2} w_{n}^{L(j)} .
\end{gathered}
$$

Para os demais $P$, precisamos calcular o comprimentos $l_{1}$ e $l_{2}$ dos lados 1 e 2 . Se $\alpha=\beta=\frac{\pi}{2 n}$, temos $\lambda=l_{1}=l_{2}=\frac{1}{w_{n}+w_{n}^{-1}}$. Mais geralmente, temos

$$
l_{1}(X)=\frac{\sin (\alpha)}{\sin (\alpha+\beta)} \text { e } l_{2}(X)=\frac{\sin (\beta)}{\sin (\alpha+\beta)} .
$$

Para calcular as derivadas das $F$ em questão, vamos precisar das derivadas parciais de $l_{1}$ e $l_{2}$ com relação a $\alpha$ e $\beta$. Temos

$$
\begin{gathered}
\lambda_{1}:=\frac{d l_{1}}{d \alpha}\left(V_{n}\right)=\frac{d l_{2}}{d \beta}\left(V_{n}\right)=\left.\frac{\sin (\beta)}{\sin ^{2}(\alpha+\beta)}\right|_{V_{n}}=\frac{2 i w_{n}}{\left(w_{n}-w_{n}^{-1}\right)\left(w_{n}+w_{n}^{-1}\right)^{2}} \\
\lambda_{2}:=\frac{d l_{1}}{d \beta}\left(V_{n}\right)=\frac{d l_{2}}{d \alpha}\left(V_{n}\right)=\left.\frac{-\cos (\alpha+\beta) \sin (\alpha)}{\sin ^{2}(\alpha+\beta)}\right|_{V_{n}}=\frac{-i w_{n}\left(w_{n}^{2}-w_{n}^{-2}\right)}{\left(w_{n}-w_{n}^{-1}\right)\left(w_{n}+w_{n}^{-1}\right)^{2}} .
\end{gathered}
$$

Os demais valores de $P$ são

$$
\begin{gathered}
P\left[a_{1}, A_{1}\right]\left(V_{n}\right)=\sum_{j=0}^{4} w_{n}^{L(j)}+l_{2}\left(V_{n}\right) w_{n}^{L(5)+1}, \\
P\left[a_{1}, A_{2}\right]\left(V_{n}\right)=\sum_{j=0}^{8} w_{n}^{L(j)}+l_{2}\left(V_{n}\right) w_{n}^{L(9)+1}, \\
P\left[a_{1}, B_{1}\right]\left(V_{n}\right)=l_{1}\left(V_{n}\right), \\
P\left[a_{1}, B_{3}\right]\left(V_{n}\right)=\sum_{j=0}^{14} w_{n}^{L(j)}+l_{2}\left(V_{n}\right) w_{n}^{L(15)-1} .
\end{gathered}
$$


Lembrando que $Q\left(V_{n}\right)=\sum_{j=0}^{19} w_{n}^{L(j)}=8 w_{n}+4 w_{n}^{3}+6 w_{n}^{-1}+2 w_{n}^{-3}$, calculamos $\tilde{F}=P \bar{Q}$ e $F=\Im(\tilde{F})$ para as 6 funções $P$ de interesse acima listadas. Depois calculamos $\frac{\partial F}{\partial \alpha}\left(V_{n}\right)$ e $\frac{\partial F}{\partial \beta}\left(V_{n}\right)$ para cada caso. Mas

$$
\frac{\partial F}{\partial \alpha}\left(V_{n}\right)=\frac{\partial \Im(\tilde{F})}{\partial \alpha}\left(V_{n}\right)=\Im\left(\frac{\partial P \bar{Q}}{\partial \alpha}\left(V_{n}\right)\right)=\Im\left(\frac{\partial P}{\partial \alpha} \bar{Q}\left(V_{n}\right)+P \frac{\partial \bar{Q}}{\partial \alpha}\left(V_{n}\right)\right) .
$$

O mesmo vale para derivada parcial com relação a $\beta$. Temos

$$
\frac{\partial \bar{Q}}{\partial \alpha}\left(V_{n}\right)=\sum_{j=0}^{19} \hat{e}_{j, 1} w_{n}^{-L(j)} \text { e } \frac{\partial \bar{Q}}{\partial \beta}\left(V_{n}\right)=\sum_{j=0}^{19} \hat{e}_{j, 2} w_{n}^{-L(j)} .
$$

Nas derivadas parciais de $P$, os termos da 3-espinha são derivados como em $\bar{Q}$ acima e os termos que dependem de $l_{1}$ e $l_{2}$ são derivados do seguinte modo:

$$
\begin{gathered}
\frac{\partial l_{2} w_{n}^{L(k) \pm 1}}{\partial \alpha}\left(V_{n}\right)=\lambda_{2} w_{n}^{L(k) \pm 1}+l_{2}\left(\hat{e}_{k, 1} \pm 1\right) w_{n}^{L(k) \pm 1} \\
\mathrm{e} \frac{\partial l_{2} w_{n}^{L(k) \pm 1}}{\partial \beta}\left(V_{n}\right)=\lambda_{1} w_{n}^{L(k) \pm 1}+l_{2}\left(\hat{e}_{k, 2} \pm 1\right) w_{n}^{L(k) \pm 1}
\end{gathered}
$$

Com isto, os autores usam o software Mathematica para manipulação simbólica das parcelas das somatórias e obtém as desigualdades $\frac{\partial H_{i, j}}{\partial \alpha}\left(V_{n}\right)<0$ e $\frac{\partial H_{i, j}}{\partial \beta}\left(V_{n}\right) \geq 0$.

\subsection{A família de palavras $W_{n, k}$}

Nesta seção definiremos as palavras $W_{n, k}$, para $n \geq 3$ e $k \geq 0$, e mostraremos o seguinte teorema.

Teorema 3.3.1. Para todo $n \geq 3$ existe $\varepsilon=\varepsilon_{n}>0$ tal que

$$
\overline{B_{++}\left(V_{n}, \varepsilon\right)} \backslash\left\{V_{n}\right\} \subset \bigcup_{k=0}^{\infty} O\left(W_{n, k}\right) .
$$

O teorema 3.3.1 será demonstrado usando o resultado a seguir.

Teorema 3.3.2. Seja $S_{n, k}$ a dilatação que leva $V_{n}$ em 0 e expande distâncias por $\zeta_{n} k^{2}$, onde $\zeta_{n}=2(n-1) \cot (\pi / 2 n) \approx 4 n^{2} / \pi$. Fixado $n$, conforme $k \rightarrow \infty$, o fecho de $S_{n, k}\left(O\left(W_{n, k}\right)\right)$ converge na métrica Hausdorff para o quadrilátero $Q_{n}$ de vértices

$$
v_{1}=\left(-\frac{1}{n}, 1-\frac{1}{n}\right), v_{2}=\left(1-\frac{1}{n},-\frac{1}{n}\right), v_{3}=\left(a_{n}, a_{n}\right) \text { e } v_{4}=\left(\mu_{n} a_{n}, \mu_{n} a_{n}\right),
$$

onde $a_{n}=\frac{1}{2}-\frac{1}{2 n}$ e $\mu_{n}=\frac{1}{2}-\frac{\tan ^{2}(\pi / 2 n)}{2}$.

A métrica Hausdorff no enunciado do teorema 3.3.2 é uma maneira de medir distância entre conjuntos. Mais precisamente, dado um espaço métrico $(M, d)$ e dois subconjuntos $X$ e $Y$ não vazios de $M$, definimos a métrica Hausdorff por

$$
\begin{aligned}
d_{h}(X, Y) & =\max \left\{\sup _{x \in X} \inf _{y \in Y} d(x, y), \sup _{y \in Y} \inf _{x \in X} d(x, y)\right\} \\
& =\inf \left\{\varepsilon \geq 0: X \subseteq Y_{\varepsilon} \text { e } Y \subseteq X_{\varepsilon}\right\}
\end{aligned}
$$


onde $X_{\varepsilon}=\bigcup_{x \in X}\{z \in M: d(x, z) \leq \varepsilon\}$. Então dizer que $\overline{S_{n, k}\left(O\left(W_{n, k}\right)\right)} \stackrel{k \rightarrow \infty}{\longrightarrow} Q_{n}$ na métrica Hausdorff é equivalente a dizer que para todo compacto $Q_{n}^{\prime} \subset Q_{n}$ existe $k^{\prime} \in \mathbb{N}$ tal que para todo $k>k^{\prime}$ temos $Q_{n}^{\prime} \subset S_{n, k}\left(O\left(W_{n, k}\right)\right)$.

Vejamos como usamos o teorema 3.3.2 para demonstrar o 3.3.1.

Demonstração de 3.3.1. Fixado $n$, o teorema 3.3 .2 nos diz que $S_{n, k}\left(O\left(W_{n, k}\right)\right)$ converge para $Q_{n}$ quando $k$ tende a infinito. Os pontos que definem o quadrilátero $Q_{n}$ são tais que $v_{1}$ está sempre no segundo quadrante, $v_{2}$ no quarto e $v_{3}$ e $v_{4}$ estão no primeiro. Além disso, este quadrilátero é simétrico com relação à reta $\alpha=\beta$. Passando pela pré-imagem de $S_{n, k}$, temos que $O\left(W_{n, k}\right)$ converge na métrica Hausdorff para $S_{n, k}^{-1}\left(Q_{n}\right)$ para todo $k$.

Logo, existem constantes $0<\varepsilon_{1}<\varepsilon_{2}$ tais que, para $k$ suficientemente grande, $O\left(W_{n, k}\right)$ contém o conjunto $V_{n}+\Lambda_{k}$, onde $\Lambda_{k}$ é o trapézio definido como o fecho convexo de $\left(\varepsilon_{1} / k^{2}, 0\right),\left(\varepsilon_{2} / k^{2}, 0\right)$, $\left(0, \varepsilon_{1} / k^{2}\right)$ e $\left(0, \varepsilon_{2} / k^{2}\right)$.

Note que, ao contrário dos pontos $v_{i}$ que definem $Q_{n}$, os pontos $\tilde{v}_{i}$ convergem pra 0 quando $k$ vai a infinito. Logo, os trapézios acima definidos se agrupam a partir de uma distância de $V_{n}$, isto é, existe $\varepsilon_{n}$ tal que

$$
\overline{B_{++}\left(V_{n}, \varepsilon\right)} \backslash\left\{V_{n}\right\} \subset \bigcup_{k=0}^{\infty} O\left(W_{n, k}\right) .
$$

Vamos tratar agora de definir indutivamente e mostrar algumas propriedades das palavras $W_{n, k}$ que serão usadas nesta seção. Note que estamos tratando de uma família bi-infinita de palavras. Quando $k$ aumenta, aumentamos o número de leques maximais do desdobramento, e quando $n$ aumenta, aumentamos o número de triângulos em cada leque. Esta definição indutiva ficará clara ao vermos sua representação na órbita reticulada quadrada e no desdobramento. Começamos com a primeira palavra da família, que tem comprimento 52

$$
W_{3,0}=23231(3) 132323131232(3) 231313232131(3) 132323213132(3) 2313131 \text {, }
$$

cujo desdobramento no triângulo $V_{3}$ pode ser visto na figura 3.20. Nesta figura, destacamos quatro arestas que ligam os pontos $a_{i}$ e $b_{i}$. Chamamos estas arestas de dobradiças do desdobramento. No desdobramento $U\left(W_{n, k}, V_{n}\right)$, estas dobradiças estão "abertas", mas ao desdobrar a mesma palavra em outro triângulo, é possível ver as "dobras". Veja a figura 3.21. Em todos os desdobramentos $U\left(W_{n, k}, V_{n}\right)$ teremos exatamente 4 dobradiças que ligam vértices superiores a vértices inferiores.

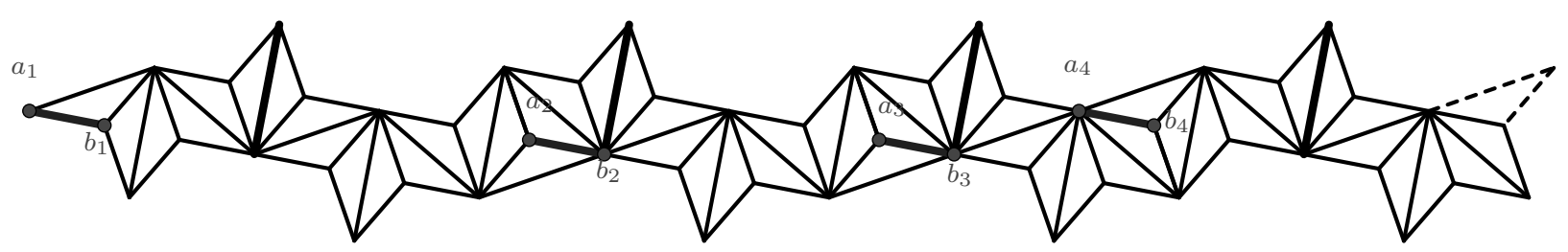

Figura 3.20: $U\left(W_{30}, V_{3}\right)$.

Na figura 3.23 vemos a representação em órbitas reticuladas de $W_{3,0}$. Note que estas órbitas são simétricas com relação à linha tracejada, e as duas "metades" se interceptam, logo vamos representar apenas uma das metades. Quando $n$ cresce uma unidade, aumentamos os lados da órbita reticulada quadrada em um quadrado de lado 2, veja a figura 3.24. Em $\hat{Q}_{3}\left(W_{3, k}\right)$ as grades são $2 \times 3$, e em $\hat{Q}_{3}\left(W_{n, k}\right)$ as grades são $(n-1) \times n$. Quando $k$ cresce, aumentamos o número de grades que a órbita vai "contornar". Em $\hat{Q}_{3}\left(W_{n, 1}\right)$ são 4 grades em cada metade e em $\hat{Q}_{3}\left(W_{n, k}\right)$ são $2(k+1)$ grades em cada metade, veja a figura 3.25 . 


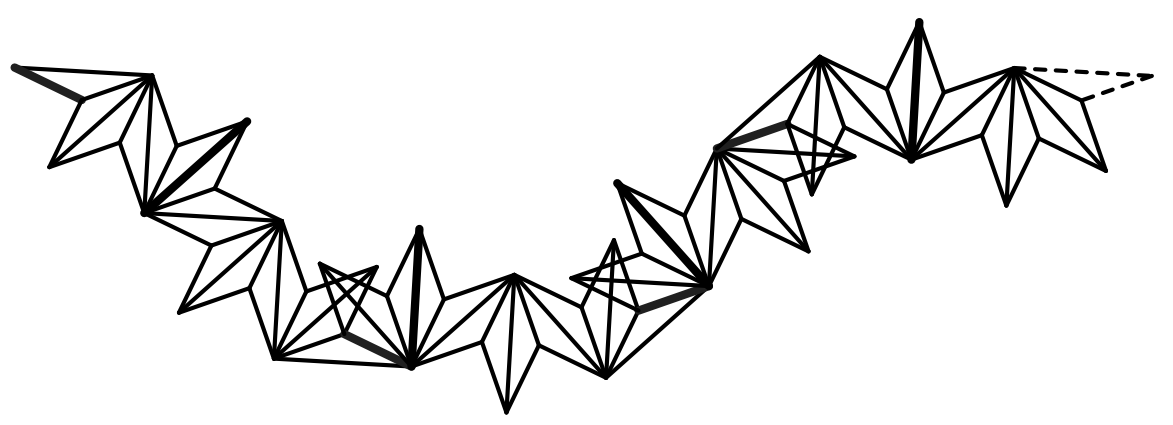

Figura 3.21: $U\left(W_{30}, V_{4}\right)$.

$W_{n, k}$ tem comprimento $24 n+30 k^{2}-68 k-20$. Dado o desdobramento de $W_{n, k}$, o desdobramento de $W_{n+1, k}$ é obtido aumentando 2 triângulos em cada leque maximal. Isso se reflete na órbita reticulada quadrada com o aumento de tamanho das grandes que aparecem, como descrito acima. O desdobramento de $W_{n, k+1}$ é obtido cortando algumas arestas e inserindo um bloco.

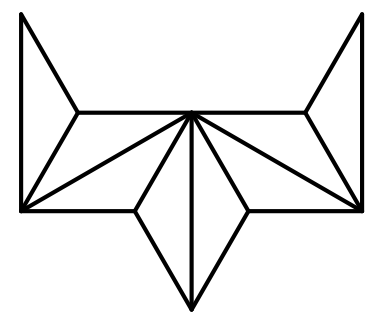

Figura 3.22: Um bloco para $W_{3, k}$.

Um bloco no desdobramento de $W_{n, k}$ é o desdobramento da palavra $(31)^{(n-1) / 2}(32)^{n-1}(31)^{(n-1) / 2}$ se $n$ for ímpar e $(13)^{(n-2) / 2} 1(32)^{n-1}(31)^{(n-2) / 2} 3$ se $n$ for par (no caso par, o bloco inserido tem a mesma forma e orientação pois o triângulo é isósceles, mas pode ter os 1's e 2's trocados). veja a figura 3.22 .

Para obter $W_{3,1}$ a partir de $W_{3,0}$, cortamos ao longo das quatro arestas quase verticais destacadas na figura 3.20 e inserimos um bloco. Destacamos com um círculo os símbolos que correspondem a aresta onde faremos o corte para inserir um bloco. Quando $n$ é ímpar, o símbolo circulado é 3, quando $n$ é par, ele pode ser 1 ou 2 em cada posição. Na palavra $W_{3,0}$, esta operação corresponde a substituir cada (3) com a palavra (3) 13232313 correspondente ao bloco 31323231.

Como já mencionamos, existem 4 arestas em $U\left(W_{n, k}, V_{n}\right)$ que conectam vértices superiores com inferiores, as 4 dobradiças. Existem mais arestas com a mesma inclinação que as dobradiças, e as chamamos de arestas quase horizontais. No total são $24+16 k$ arestas quase horizontais. Também

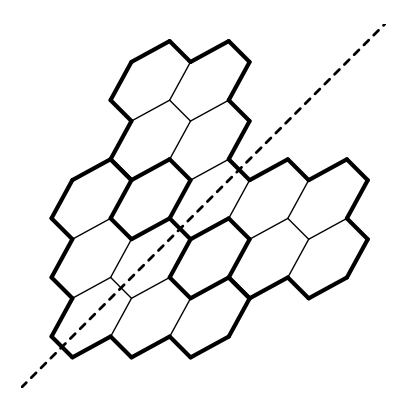

(a) $H\left(W_{3,0}\right)$

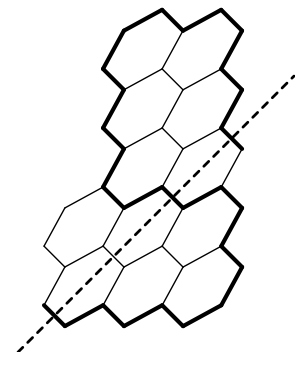

(b) Metade de $H\left(W_{3,0}\right)$ com a (c) Metade de $\hat{Q}_{3}\left(W_{3,0}\right)$ com a linha de simetria.

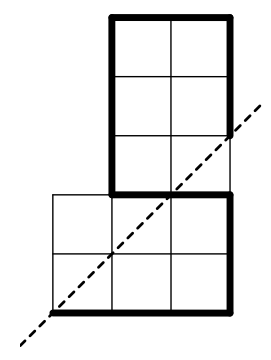

linha de simetria.

Figura 3.23: A órbita reticulada quadrada $\hat{Q}_{3}\left(W_{3,0}\right)$ é baseada em duas grades $2 \times 3$. 


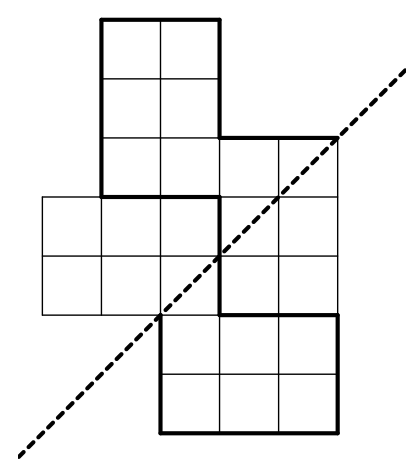

(a) $\hat{Q}_{3}\left(W_{3,1}\right)$

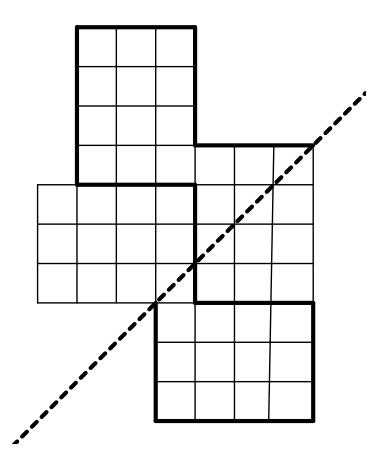

(b) $\hat{Q}_{3}\left(W_{4,1}\right)$

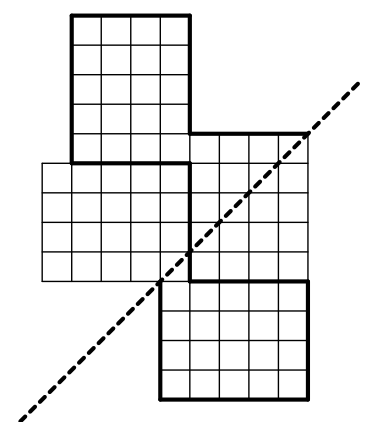

(c) $\hat{Q}_{3}\left(W_{5,1}\right)$

Figura 3.24: Metade da órbita reticulada quadrada $\hat{Q}_{3}\left(W_{n, 1}\right) \operatorname{com} n=3,4,5$.

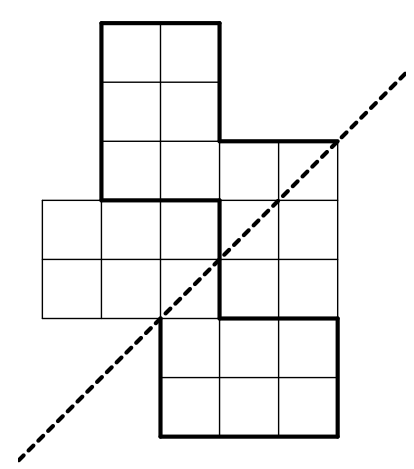

(a) $\hat{Q}_{3}\left(W_{3,1}\right)$

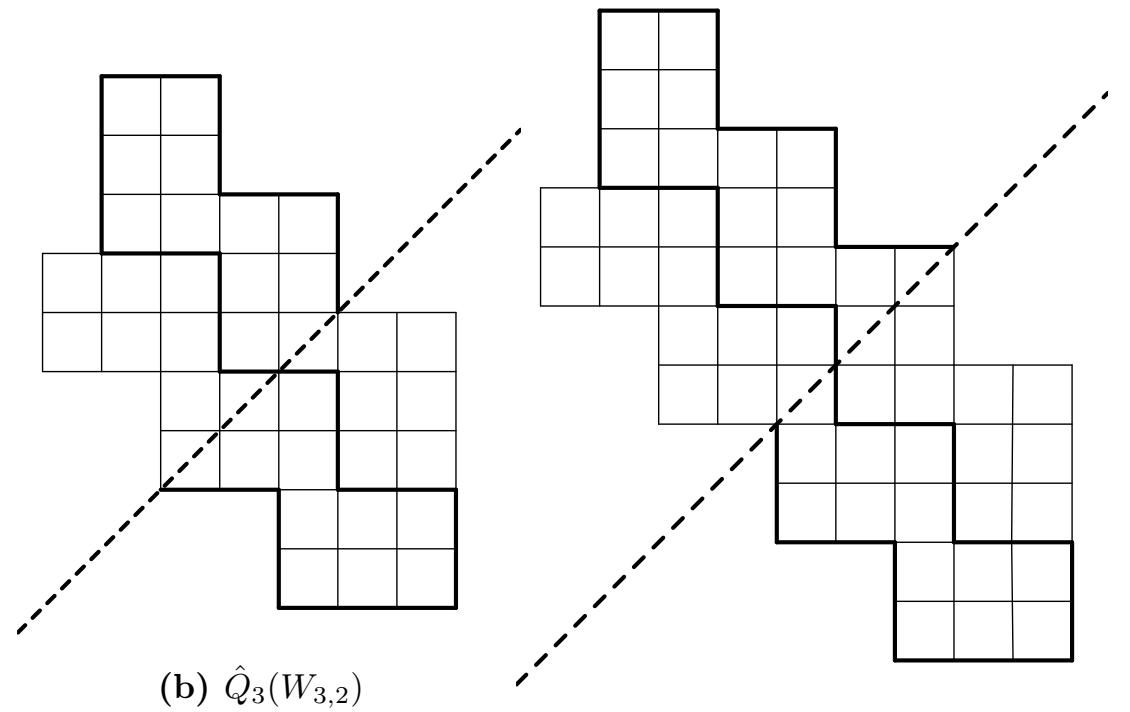

(c) $\hat{Q}_{3}\left(W_{3,3}\right)$

Figura 3.25: Metade da órbita reticulada quadrada $\hat{Q}_{3}\left(W_{3, k}\right) \operatorname{com} k=1,2,3$. 
existem $12+8 k$ arestas quase verticais todas com a mesma inclinação, como as que cortamos para inserir blocos. Cada aresta quase vertical é flanqueada por duas arestas quase horizontais, uma é reflexão da outra.

Isto ocorre pois a junção dos leques em $U\left(W_{n, k}, V_{n}\right)$ forma ângulos retos, como pode ser observado na figura 3.20 e estendido para $U\left(W_{n, k}, V_{n}\right)$. Note que numa vizinhança de $V_{n}$, estas famílias de arestas deixam de ser paralelas, mas fixamos nomenclatura delas para $V_{n}$ e mantemos os nomes para triângulos próximos.

Definição 3.3.1. Seja $A_{n}$ o quadrado no espaço dos parâmetros $\Delta$ com vértices em $V_{n}$ e $V_{n-1} e$ $R_{n, k}^{\prime} \subset A_{n}$ o subconjunto dos pontos $X$ tais que as arestas quase horizontais de $U\left(W_{n, k}, X\right)$ têm inclinação negativa. A região pivô da palavra $W_{n, k}$, denotada por $R_{n, k}$, é a componente conexa de $R_{n, k}^{\prime}$ que contém $V_{n}$.

O lema a seguir é essencial para a demonstração do teorema 3.3.2, pois ele permite determinar o ladrilho de órbita $O\left(W_{n, k}\right)$ usando funções comparadoras para 16 combinações de pontos, independente de quão grandes sejam $n$ e $k$.

Lema 5. Seja $X \in R_{n, k}$ um ponto do espaço $\Delta$ de parâmetros dos triângulos obtusos. Se em $U\left(W_{n, k}, X\right)$ todos os vértices superiores $a_{1}, a_{2}, a_{3}$ e $a_{4}$ ligados às dobradiças estão acima de todos os vértices inferiores $b_{1}, b_{2}, b_{3}$ e $b_{4}$ ligados às dobradiças, então $X \in O\left(W_{n, k}\right)$.

Demonstração. Queremos mostrar que todos os vértices base superiores estão acima de todos os vértices base inferiores de $U\left(W_{n, k}, X\right)$, assim, pelo lema do leque (lema 2), concluímos que $X \in$ $O\left(W_{n, k}\right)$.

Seja $v$ um vértice base superior que não é $a_{1}, a_{2}, a_{3}$ ou $a_{4}$. $v$ satisfaz uma das seguintes condições

1. $v$ é o vértice esquerdo de uma aresta quase horizontal;

2. $v$ é a reflexão por uma aresta quase vertical de um vértice $v^{\prime}$ que satisfaz 1.

De fato, isto vale para $W_{3,0}$ e continua verdade quando aumentamos o número de triângulos ou adicionamos blocos para formar o desdobramento $W_{n, k}$. Como $X \in R_{n, k}$, as arestas quase horizontais têm inclinação negativa. Além disso, como as arestas quase verticais têm inclinação positiva para $V_{n}$ e são flanqueadas simetricamente por duas arestas quase horizontais de inclinação negativa, então para $X \in R_{n, k}$, as arestas quase verticais continuam com inclinação positiva. Logo, $v$ está acima de $v^{\prime}$.

Como o argumento acima é válido para todo vértice base superior que não é $a_{1}, a_{2}, a_{3}$ ou $a_{4}$, vemos que o vértice base superior mais baixo deve estar entre $a_{1}, a_{2}, a_{3}$ e $a_{4}$. De maneira análoga, vemos que o vértice base inferior mais alto deve estar entre $b_{1}, b_{2}, b_{3}$ e $b_{4}$. Como $a_{1}, a_{2}, a_{3}$ e $a_{4}$ estão acima de $b_{1}, b_{2}, b_{3}$ e $b_{4}$, todos os vértices base superiores estão acima de todos os vértices base inferiores.

Fixado $n$, essencialmente, o teorema que queremos mostrar diz qual é a forma do ladrilho de órbita $O\left(W_{n, k}\right)$ quando $k$ vai a infinito. Sabemos agora que a região $O\left(W_{n, k}\right)$ de $\Delta$ é limitada com as curvas de nível em 0 de 16 funções analíticas. Denotamos por $F_{k}$ a função comparadora da palavra $W_{n, k}$ quando $n$ é fixado, as 16 funções de interesse são $F_{k}\left[a_{i}, b_{j}\right](X) \operatorname{com}(i, j) \in\{1,2,3,4\}^{2}$.

Estabelecidas as propriedades das palavras $W_{n, k}$ e o lema 5, falta enunciar dois resultados que serão aplicados para concluir a demonstração de 3.3.2. Para isso, vamos introduzir mais alguns conceitos a seguir.

Assim como na demonstração do teorema 3.2.1, o teorema 3.3.2 foi verificado pelos autores em [HS09] experimentalmente usando recursos computacionais, e depois foi demonstrado rigorosamente. Hooper e Schwartz optaram por mostrar os resultados auxiliares utilizados na maior generalidade possível, assim como adaptar os cálculos para inteiros e manipulação simbólica computacional. Por estes motivos, o leitor pode sentir um certo estranhamento nas seções seguintes, mas a leitura até o fim provará a utilidade de certos conceitos. 


\section{Teorema de redimensionamento quadrático}

Para uma palavra qualquer, lembramos da definição de $Q: \mathbb{R}^{2} \rightarrow \mathbb{C}, Q(X)=$ $\sum_{j=0}^{m}(-1)^{j} \exp \left(i\left\langle X, \hat{e}_{j}\right\rangle\right)$. Como o suporte de $Q$ é compacto, podemos pensar em uma transformada de Fourier $\hat{Q}$ de $Q$ indexada pelos inteiros, que satisfaz

$$
Q(X)=\sum_{V \in \mathbb{Z}^{2}} \hat{Q}(V) \exp (i\langle X, V\rangle)
$$

onde $\hat{Q}(V)$ tem suporte finito. Para mais detalhes, veja a seção 2.5 de [HS09].

Para $n$ fixado, vamos lidar com famílias infinitas de funções comparadoras $\left\{F_{k}\right\}$ para cada par de pontos. Para já lidar com o fato de que no teorema 3.3.2 levarmos o ponto $V_{n}$ em 0 , vamos trabalhar com

$$
F_{k}[a, b](X)=\Im\left(P_{k}[a, b]\left(X+V_{n}\right) \overline{Q_{k}\left(X+V_{n}\right)}\right) .
$$

Seja $G_{k}(X)=F_{k}\left(X k^{-2}\right)$ a $F$ reescalada. Queremos encontrar condições sobre as famílias $\left\{P_{k}\right\}$ e $\left\{Q_{k}\right\}$ de forma que a família $\left\{G_{k}\right\}$ converge para uma função linear. Isto dará a forma de losango para o ladrilho de órbita.

Seja $T: \mathbb{Z}^{2} \rightarrow \mathbb{Z}^{2}$ a translação definida por $T\left(x_{1}, x_{2}\right)=\left(x_{1}+(2 n-2), x_{2}-(2 n-2)\right)$ e $\left\{R_{k}\right\}$ uma das famílias $\left\{P_{k}\right\}$ ou $\left\{Q_{k}\right\}$. Podemos escrever $R_{k}$ em função de sua transformada de Fourier $\hat{R}_{k}: \mathbb{Z}^{2} \rightarrow \mathbb{Z}:$

$$
R_{k}(X)=\sum_{V \in \mathbb{Z}^{2}} \hat{R}_{k}(V) \exp (i\langle X, V\rangle)
$$

Definição 3.3.2. A família $\left\{\hat{R}_{k}\right\}$ tem crescimento linear se existe uma função $\hat{R}^{\#}: \mathbb{Z}^{2} \rightarrow \mathbb{Z}$ tal que

$$
\hat{R}_{k+1}=\hat{R}_{k}+\hat{R}^{\#} \circ T^{k},
$$

com $k=0,1,2, \ldots$ Dizemos que $\hat{R}^{\#}$ é um gerador de crescimento para $\left\{R_{k}\right\}$.

Temos

$$
R^{\#}(X)=\sum_{V \in \mathbb{Z}^{2}} \hat{R}^{\#}(V) \exp (i\langle X, V\rangle)
$$

Intuitivamente falando, uma família tem crescimento linear se a órbita reticulada quadrada de seus elementos cresce no sentido da translação $T$. Veja na figura 3.25 que isto ocorre com $W_{n, k}$ quando $k$ cresce.

Vamos supor que $\left\{\hat{P}_{k}\right\}$ e $\left\{\hat{Q}_{k}\right\}$ tem crescimento linear. Definimos

$$
\begin{gathered}
\delta:=\operatorname{det}\left(\begin{array}{cc}
P_{0}\left(V_{n}\right) & P^{\#}\left(V_{n}\right) \\
Q_{0}\left(V_{n}\right) & Q^{\#}\left(V_{n}\right)
\end{array}\right), \\
\delta_{j}:=\operatorname{det}\left(\begin{array}{cc}
P^{\#}\left(V_{n}\right) & \partial_{j} P^{\#}\left(V_{n}\right) \\
Q^{\#}\left(V_{n}\right) & \partial_{j} Q^{\#}\left(V_{n}\right)
\end{array}\right), \\
\text { e } \Delta_{j}:=(-1)^{j+1}(n-1) \delta+\Im\left(\delta_{j}\right),
\end{gathered}
$$

onde $\partial_{1}$ é a derivada parcial com relação a $\alpha$ e $\partial_{2}$ é a derivada parcial com relação a $\beta$.

Teorema 3.3.3 (Teorema de redimensionamento quadrático). Suponha que as famílias $\left\{\hat{P}_{k}\right\}$ e $\left\{\hat{Q}_{k}\right\}$ tem crescimento linear com relação à translação $T$ e que $P^{\#}\left(V_{n}\right), Q^{\#}\left(V_{n}\right)$ e $\delta$ são reais. Então $\left\{G_{k}\right\}$ converge na topologia $C^{\infty}$ para $G$, que é dada por

$$
G(\alpha, \beta)=F_{0}(0,0)-\Delta_{1} \alpha-\Delta_{2} \beta .
$$


A convergência do teorema acima significa que todas as derivadas parciais de $G_{k}$ convergem para as derivadas correspondentes de $G$.

Para a demonstração do teorema 3.3 .3 veja a seção 6 de [HS09].

O sentido deste teorema, especialmente no contexto em que ele será aplicado, é o seguinte: se uma família infinita de funções comparadoras $\left\{F_{k}\right\}$ cresce sempre na mesma direção de maneira padronizada, então conseguimos calcular o limite destas funções comparadoras calculadas em qualquer ponto $X$ usando a primeira delas no ponto $V_{n}$ e constantes calculadas com base nas primeiras $P, Q$ e seus geradores de crescimento.

\section{Lema pivô}

Seja $T_{n, k}$ a dilatação que leva $V_{n}$ para $(0,0)$ e dilata distâncias a um fator de $k^{2}$. Seja $\zeta_{n}=$ $2(n-1) \cot (\pi / 2 n) \approx 4 n^{2} / \pi$ como no teorema 3.3.2.

Lema 6 (Lema pivô). Para todo $n$, o conjunto $T_{n, k}\left(R_{n, k}\right)$ converge, conforme $k$ vai a infinito, para a faixa $\Sigma_{n}$ definida por $|\alpha-\beta|<\frac{1}{\zeta_{n}}$.

A demonstração deste lema está na seção 7 de [HS09].

Note que $\zeta_{n} T_{n, k}=S_{n, k}$.

\section{Demonstração de 3.3.2}

Queremos estudar a forma dos conjuntos $T_{n, k}\left(O\left(W_{n, k}\right)\right)$ conforme $k$ tende a infinito. Como já mencionamos, $X \in O\left(W_{n, k}\right)$ se $a_{1}, a_{2}, a_{3}$ e $a_{4}$ estão acima de $b_{1}, b_{2}, b_{3}$ e $b_{4}$. Seja $(p, q)$ qualquer par entre estes vértices e $F_{k}[p, q](X)$ a função comparadora entre esses dois pontos para a palavra $W_{n, k}$ com $n$ fixado.

Se $\left\{F_{k}[p, q]\right\}$ satisfaz as hipóteses do teorema 3.3.3, seja $G[p, q]$ o limite reescalado de $\left\{F_{k}[p, q]\right\}$. Seja $\Omega_{n} \in \Delta$ o conjunto dos pontos tal que $G\left[a_{i}, b_{j}\right]<0$. Pelo teorema 3.3.3, $G\left[a_{i}, b_{j}\right]$ é linear e $\Omega_{n}$ é um polígono com no máximo 16 lados. Além disso, $\Omega_{n}$ está contido em $\Sigma_{n}$ pois $\Sigma_{n}=$ $\lim _{k \rightarrow \infty} T_{n, k}\left(R_{n, k}\right)$ e $R_{n, k}$ é o conjunto dos pontos com dobradiça de inclinação negativa, isto é, $\Sigma_{n}$ é definido por $G\left[a_{i}, b_{i}\right]<0$.

Vejamos a seguir que $T_{n, k}\left(O\left(W_{n, k}\right)\right)$ converge na métrica Hausdorff para $\Omega_{n}$ e depois vamos verificar que $\left\{F_{k}[p, q]\right\}$ de fato satisfaz as hipóteses do teorema 3.3.3.

Vamos mostrar que $O\left(W_{n, k}\right)$ converge na métrica Hausdorff para $T_{n, k}^{-1}\left(\Omega_{n}\right)$. Isto é equivalente a, para todo compacto $V \subset T_{n, k}^{-1}\left(\Omega_{n}\right)$, existir $k^{\prime} \in \mathbb{N}$ tal que para todo $k>k^{\prime}$ temos $V \subset O\left(W_{n, k}\right)$. Note que $T_{n, k}^{-1}\left(\Omega_{n}\right)$ está contido em $R_{n, k}$ pois $\Omega_{n} \subset \Sigma_{n}$.

Seja $U$ um aberto qualquer cujo fecho está contido no interior de $\Omega_{n}$. Como $\Omega_{n}$ é limitado, $\bar{U}$ é compacto. Assim, $T_{n, k}^{-1}(U)$ também tem fecho compacto em $R_{n, k}$.

Seja $X \in T_{n, k}^{-1}(U)$. Pela demonstração do lema 5 , o vértice base superior mais baixo de $\left(W_{n, k}, X\right)$ está entre $a_{1}, a_{2}, a_{3}$ e $a_{4}$ e o vértice base inferior mais alto está entre $b_{1}, b_{2}, b_{3}$ e $b_{4}$. Como $\Omega_{n}$ é o conjunto dos pontos tal que $G\left[a_{i}, b_{j}\right]<0$, para $k$ suficientemente grande, $G_{k}\left[a_{i}, b_{j}\right]<0$ em $U$. Logo, $F_{k}\left[a_{i}, b_{j}\right](X)<0$ para $X$ suficientemente grande. Portanto $X$ satisfaz as hipóteses do lema 5 e $X \in O\left(W_{n, k}\right)$. Portanto $V \subset O\left(W_{n, k}\right)$.

Fixado $n$, conforme $k$ cresce, a órbita reticulada quadrada "cresce" na direção da reta $x_{2}=-x_{1}$ (lembre-se da figura 3.25), por isso, as famílias $\left\{Q_{k}\right\}$ e $\left\{F_{k}[p, q]\right\}$ têm crescimento linear com relação com relação a $T$.

O fato das famílias $\left\{F_{k}\left[a_{i}, b_{i}\right]\right\}$ satisfazerem as hipóteses do teorema 3.3.3 é consequência da demonstração do lema 6 e temos, $\operatorname{com} c=\cos (\pi / 2 n)$ e $s=\sin (\pi / 2 n)$,

$$
G\left[a_{1}, b_{1}\right](\alpha, \beta)=G\left[a_{3}, b_{3}\right](\alpha, \beta)=-8 c s-16(n-1) c^{2} \alpha+16(n-1) c^{2} \beta
$$




$$
\text { e } G\left[a_{2}, b_{2}\right](\alpha, \beta)=G\left[a_{4}, b_{4}\right](\alpha, \beta)=-8 c s+16(n-1) c^{2} \alpha-16(n-1) c^{2} \beta .
$$

Esta simetria nas variáveis $\alpha$ e $\beta$ de $G$ é explicada pela simetria dos vértices da órbita reticulada quadrada que representam as dobradiças. Veja a seção 7 de [HS09].

Vamos mostrar que as famílias

(I) $\left\{F_{k}\left[a_{1}, b_{2}\right]\right\}$

(II) $\left\{F_{k}\left[b_{1}, b_{2}\right]\right\}$

(III) $\left\{F_{k}\left[b_{3}, b, 4\right]\right\}$

também satisfazem as hipóteses do teorema de redimensionamento quadrático. Assim, como qualquer função $F_{k}[p, q]$ pode ser escrita como combinação linear destas sete funções, teremos que $\left\{F_{k}[p, q]\right\}$ satisfaz as hipóteses do teorema 3.3.3.

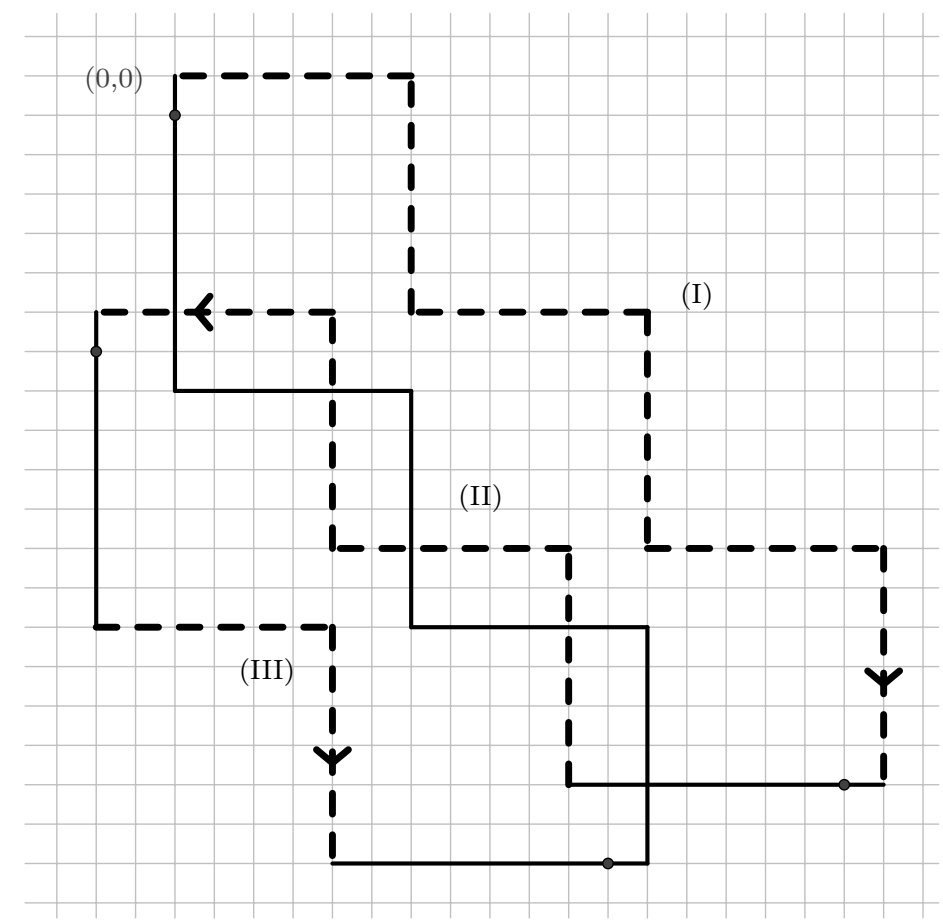

Figura 3.26: Ilustração dos caminhos de P para as famílias (I), (II), (III) na órbita reticulada quadrada $\hat{Q}_{3}\left(W_{4,1}\right)$.

Note que na figura 3.26 não estamos destacando os quadrados de lado 2 da órbita reticulada quadrada, e sim a grade gerada por $\mathbb{Z}^{2}$. Faremos isto para destacar alguns pontos. Por exemplo, os pontos cinza são correspondentes às arestas que são dobradiças.

Vamos primeiro tratar da família $\left\{Q_{k}\right\}$ e, para isso, calcular $Q_{0}$ e $Q^{\#}$.

Os doze vértices da órbita reticular quadrada de $W_{n, 0}$ na ordem em que são percorridos, são $\hat{e}_{0}=(0,1), \hat{e}_{1}=(2 n-2,1), \hat{e}_{2}=(2 n-2,-2 n+3), \hat{e}_{3}=(4 n-4,-2 n+3), \hat{e}_{4}=(4 n-4,-4 n+5)$, $\hat{e}_{5}=(2 n-4,-4 n+5), \hat{e}_{6}=(2 n-4,-2 n+3), \hat{e}_{7}=(-2,-2 n+3), \hat{e}_{8}=(-2,-4 n+3), \hat{e}_{9}=$ $(2 n-2,-4 n+3), \hat{e}_{10}=(2 n-2,-2 n+1)$ e $\hat{e}_{11}=(0,-2 n+1)$.

Os oito vértices do suporte de $\hat{Q}^{\#}$ são $\hat{q}_{0}=(6 n-6,-4 n+5), \hat{q}_{1}=(6 n-6,-6 n+7), \hat{q}_{2}=$ $(4 n-6,-6 n+7), \hat{q}_{3}=(4 n-6,-4 n+5), \hat{q}_{4}=(2 n-4,-4 n+5), \hat{q}_{5}=(2 n-4,-6 n+5)$, $\hat{q}_{6}=(4 n-4,-6 n+5)$ e $\hat{q}_{7}=(4 n-4,-2 n+3)$.

Seja

$$
c=\cos \left(\frac{\pi}{2 n}\right) \text { e } s=\sin \left(\frac{\pi}{2 n}\right) .
$$




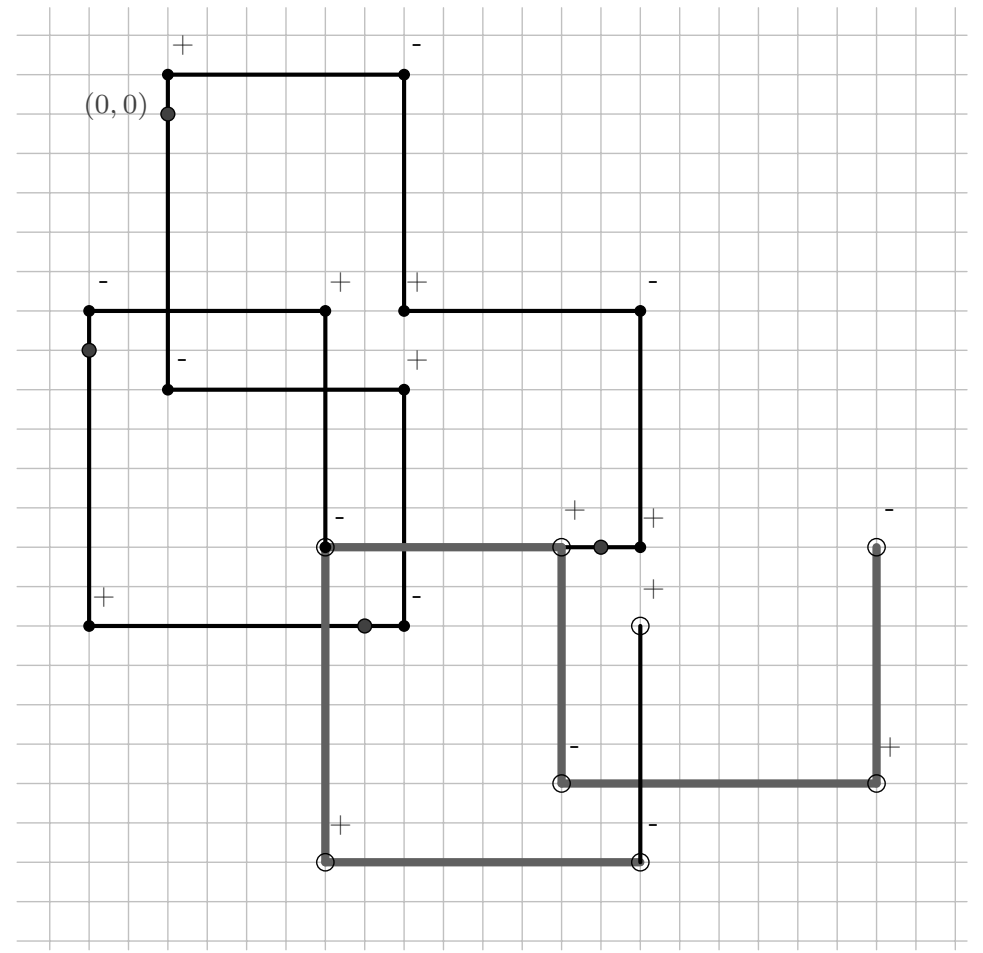

Figura 3.27: O caminho preto é $\hat{Q}_{3}\left(W_{4,0}\right)$ e o cinza é o caminho que liga os pontos do suporte de $\hat{Q}^{\#}$. Estes pontos estão ilustrados pelo círculo sem preenchimento.

Usando a lista de vértices acima, temos que

$$
\begin{gathered}
Q_{0}\left(V_{n}\right)=12 c+4 i s ; \\
Q^{\#}\left(V_{n}\right)=8 c ; \\
\delta_{1} Q^{\#}\left(V_{n}\right)=8 i(4 n-5) c ; \\
\delta_{2} Q^{\#}\left(V_{n}\right)=8(n-1) s-40 i(n-1) c .
\end{gathered}
$$

Agora tratemos da família (I) $\left\{F_{k}\left[a_{1}, b_{2}\right]\right\}$

$\mathrm{O}$ caminho de $P$ passa pelos vértices $\hat{e}_{0}=(0,1), \hat{e}_{1}=(2 n-2,1), \hat{e}_{2}=(2 n-2,-2 n+3)$, $\hat{e}_{3}=(4 n-4,-2 n+3), \hat{e}_{4}=(4 n-4,-4 n+5)$ e o de $P^{\#}$ passa pelos vértices $\hat{q}_{0}=(6 n-6,-4 n+5)$, $\hat{q}_{1}=(6 n-6,-6 n+7)$. Assim, temos que

$$
\begin{gathered}
P_{0}\left[a_{1}, b_{2}\right]\left(V_{n}\right)=5 c+i s ; \\
P^{\#}\left[a_{1}, b_{2}\right]\left(V_{n}\right)=2 c ; \\
\delta_{1} P^{\#}\left[a_{1}, b_{2}\right]\left(V_{n}\right)=12 i(n-1) c ; \\
\delta_{2} P^{\#}\left[a_{1}, b_{2}\right]\left(V_{n}\right)=2(n-1) s-2 i(5 n-6) c .
\end{gathered}
$$

Vamos calcular as constantes do teorema de redimensionamento quadrático.

$$
F_{0}\left[a_{1}, b_{2}\right](0,0)=\Im(5 c+i s)(12 c-4 i s)=-8 c s,
$$


é menor que 0 pois $a_{1}$ está acima de $b_{2}$ em $V_{n}$ para a palavra $W_{n, 0}$.

$$
\begin{gathered}
\delta=\operatorname{det}\left(\begin{array}{cc}
5 c+i s & 2 c \\
12 c+4 i s & 8 c
\end{array}\right)=16 c^{2}, \\
\Im\left(\delta_{1}\right)=\Im\left|\begin{array}{cc}
2 c & 12 i(n-1) c \\
8 c & 8 i(4 n-5) c
\end{array}\right|=-16 c^{2}(2 n-1), \\
\Im\left(\delta_{2}\right)=\Im\left|\begin{array}{cc}
2 c & 2(n-1) s-2 i(5 n-6) c \\
8 c & 8(n-1) s-40 i(n-1) c
\end{array}\right|=-16 c^{2}, \\
\Delta_{1}=\Delta_{2}=-16 n c^{2} .
\end{gathered}
$$

Logo,

$$
G\left[a_{1}, b_{2}\right](\alpha, \beta)=-8 c s+16 n c^{2} \alpha+16 n c^{2} \beta .
$$

Do mesmo modo, calculamos as constantes para $P\left[b_{2}, b_{3}\right]$ e $P\left[b_{3}, a_{4}\right]$ e temos

$$
\begin{gathered}
G\left[b_{2}, b_{3}\right](\alpha, \beta)=-16 c^{2} \alpha+16 c^{2} \beta, \\
G\left[b_{3}, a_{4}\right](\alpha, \beta)=8 c s-16 n c^{2} \alpha-16 n c^{2} \beta .
\end{gathered}
$$

Então temos o valor de $G[p, q]$ para $\left[a_{1}, b_{1}\right],\left[a_{2}, b_{2}\right],\left[a_{3}, b_{3}\right],\left[a_{4}, b_{4}\right],\left[a_{1}, b_{2}\right]$, $\left[b_{2}, b_{3}\right]$ e $\left[b_{3}, a_{4}\right]$. Queremos escrever $G[p, q]$, com $p \in\left\{a_{1}, a_{2}, a_{3}, a_{4}\right\}$ e $q \in\left\{b_{1}, b_{2}, b_{3}, b_{4}\right\}$, como uma combinação linear destas sete $G$ 's já calculadas. Porém, as quatro primeiras foram calculadas usando a diferença entre $p$ e $q$ multiplicada por uma constante que depende do comprimento de um lado do tipo 1 ou 2 , enquanto as três últimas foram calculadas usando esta diferença multiplicada por uma constante que depende do comprimento do lado 3, já que usamos só a 3-espinha. Assim, vejamos as contantes dessa combinação linear.

Definimos

$$
\tilde{G}=4 c^{2} s^{2} G
$$

se $G$ foi calculada usando a 3-espinha e

$$
\tilde{G}=s^{2} G,
$$

se $G$ foi calculada usando lados 1 ou 2. Veja a seção 8.4 de [HS09] para o calculo das constantes.

Com isso, vamos escrever nossas funções como vetores num espaço de base $\{1, \alpha, \beta\}$. Temos

$$
\begin{gathered}
\tilde{G}\left[a_{1}, b_{1}\right](\alpha, \beta)=\tilde{G}\left[a_{3}, b_{3}\right](\alpha, \beta)=\left[\begin{array}{c}
-8 c s^{3} \\
-16(n-1) c^{2} s^{2} \\
16(n-1) c^{2} s^{2}
\end{array}\right], \\
\tilde{G}\left[a_{2}, b_{2}\right](\alpha, \beta)=\tilde{G}\left[a_{4}, b_{4}\right](\alpha, \beta)=\left[\begin{array}{c}
-8 c s^{3} \\
16(n-1) c^{2} s^{2} \\
-16(n-1) c^{2} s^{2}
\end{array}\right], \\
\tilde{G}\left[a_{1}, b_{2}\right](\alpha, \beta)=-\tilde{G}\left[b_{3}, a_{4}\right](\alpha, \beta)=\tilde{G}\left[a_{4}, b_{3}\right](\alpha, \beta)=\left[\begin{array}{c}
-32 c^{3} s^{3} \\
64 n c^{4} s^{2} \\
64 n c^{4} s^{2}
\end{array}\right], \\
\text { e } \tilde{G}\left[b_{2}, b_{3}\right](\alpha, \beta)=\left[\begin{array}{c}
0 \\
-64 c^{4} s^{2} \\
64 c^{4} s^{2}
\end{array}\right] .
\end{gathered}
$$

Com isso, faltam 10 das 16 funções $\tilde{G}[p, q]$ com $p \in\left\{a_{1}, a_{2}, a_{3}, a_{4}\right\}$ e $q \in\left\{b_{1}, b_{2}, b_{3}, b_{4}\right\}$ e as calculamos usando as funções acima 


$$
\begin{aligned}
& \tilde{G}\left[a_{1}, b_{3}\right]=\tilde{G}\left[a_{1}, b_{2}\right]+\tilde{G}\left[b_{2}, b_{3}\right], \\
& \tilde{G}\left[a_{4}, b_{2}\right]=\tilde{G}\left[a_{4}, b_{3}\right]-\tilde{G}\left[b_{2}, b_{3}\right], \\
& \tilde{G}\left[a_{1}, b_{4}\right]=\tilde{G}\left[a_{1}, b_{3}\right]-\tilde{G}\left[a_{4}, b_{3}\right]+\tilde{G}\left[a_{4}, b_{4}\right], \\
& \tilde{G}\left[a_{2}, b_{1}\right]=\tilde{G}\left[a_{2}, b_{2}\right]-\tilde{G}\left[a_{1}, b_{2}\right]+\tilde{G}\left[a_{1}, b_{1}\right], \\
& \tilde{G}\left[a_{2}, b_{3}\right]=\tilde{G}\left[a_{2}, b_{2}\right]+\tilde{G}\left[b_{2}, b_{3}\right], \\
& \tilde{G}\left[a_{2}, b_{4}\right]=\tilde{G}\left[a_{2}, b_{3}\right]-\tilde{G}\left[a_{4}, b_{3}\right]+\tilde{G}\left[a_{4}, b_{4}\right], \\
& \tilde{G}\left[a_{3}, b_{1}\right]=\tilde{G}\left[a_{1}, b_{1}\right]-\tilde{G}\left[a_{1}, b_{3}\right]+\tilde{G}\left[a_{3}, b_{3}\right], \\
& \tilde{G}\left[a_{3}, b_{2}\right]=\tilde{G}\left[a_{3}, b_{3}\right]-\tilde{G}\left[b_{2}, b_{3}\right], \\
& \tilde{G}\left[a_{3}, b_{4}\right]=\tilde{G}\left[a_{3}, b_{3}\right]-\tilde{G}\left[a_{4}, b_{3}\right]+\tilde{G}\left[a_{4}, b_{4}\right], \\
& \tilde{G}\left[a_{4}, b_{1}\right]=\tilde{G}\left[a_{1}, b_{1}\right]-\tilde{G}\left[a_{1}, b_{4}\right]+\tilde{G}\left[a_{4}, b_{4}\right] .
\end{aligned}
$$

Com isso, chegamos que as 16 funções de interesse são combinação linear de apenas quatro delas: $\tilde{G}\left[a_{1}, b_{3}\right], \tilde{G}\left[a_{4}, b_{2}\right], \tilde{G}\left[a_{2}, b_{4}\right]$ e $\tilde{G}\left[a_{3}, b_{1}\right]$. Os 0 destas quatro funções delimitam a fronteira de $\Omega_{n}=\lim _{k \rightarrow \infty} T_{n, k}\left(O\left(W_{n, k}\right)\right)$ que é a região onde todas as $G[p, q]$ são negativas. De fato, lembrese que $T_{n, k}$ é a transformação que leva $V_{n}$ para $(0,0)$ e dilata distâncias a um fator de $k^{2}$ e que definimos $G_{k}(X)=F_{k}\left(X k^{-2}\right)$ com a $F_{k}(X)=\Im\left(P_{k}\left(X+V_{n}\right) \overline{Q_{k}\left(X+V_{n}\right)}\right)$.

Temos

$$
\begin{gathered}
\tilde{G}\left[a_{1}, b_{3}\right]=\left[\begin{array}{c}
-32 c^{3} s^{3} \\
64(n-1) c^{4} s^{2} \\
64(n+1) c^{4} s^{2}
\end{array}\right], \\
\tilde{G}\left[a_{4}, b_{2}\right]=\left[\begin{array}{c}
-32 c^{3} s^{3} \\
64(n+1) c^{4} s^{2} \\
64(n-1) c^{4} s^{2}
\end{array}\right], \\
\tilde{G}\left[a_{2}, b_{4}\right]=\left[\begin{array}{c}
-16 c s^{3}+32 c^{3} s^{3} \\
-64(n+1) c^{4} s^{2}+32 c^{2} s^{2}(n-1) \\
-64(n-1) c^{4} s^{2}-32 c^{2} s^{2}(n-1)
\end{array}\right], \\
\tilde{G}\left[a_{3}, b_{1}\right]=\left[\begin{array}{c}
-16 c s^{3}+32 c^{3} s^{3} \\
-64(n-1) c^{4} s^{2}-32 c^{2} s^{2}(n-1) \\
-64(n+1) c^{4} s^{2}+32 c^{2} s^{2}(n-1)
\end{array}\right] .
\end{gathered}
$$

Note que estas funções tem o segundo e o terceiro coeficientes trocados, isto é, $\tilde{G}\left[a_{4}, b_{2}\right](\alpha, \beta)=$ $\tilde{G}\left[a_{1}, b_{3}\right](\beta, \alpha)$ e $\tilde{G}\left[a_{3}, b_{1}\right](\alpha, \beta)=\tilde{G}\left[a_{4}, b_{2}\right](\beta, \alpha)$. Como queremos a região delimitada pelos pontos onde estas funções são 0 , podemos multiplicá-las por constantes. Se $\sigma=1 /\left(2 c^{2}-1\right)$. Isso nos dá

$$
\begin{gathered}
\tilde{G}\left[a_{1}, b_{3}\right] \propto\left[\begin{array}{c}
1 \\
-2(n-1)(c / s) \\
-2(n+1)(c / s)
\end{array}\right], \\
\tilde{G}\left[a_{2}, b_{4}\right] \propto\left[\begin{array}{c}
-1 \\
2(c / s)(n+1+\sigma) \\
2(n-1)(c / s)(1+2 \sigma)
\end{array}\right] .
\end{gathered}
$$

Para concluir o teorema, basta multiplicar a região $\Omega_{n}$ definida por estas quatro funções por $\zeta_{n}$ (pois $\zeta_{n} T_{n, k}=S_{n, k}$ ) e verificar que esta região é o quadrilátero do enunciado. Mas, para isso, basta dividir o segundo e o terceiro coeficientes por $\zeta_{n}$. 


\section{Capítulo 4}

\section{Condição homológica para estabilidade de órbitas bilhares em triângulos}

Dado um triângulo $T$, o refletimos pelo seu maior lado e colamos os lados correspondentes, como na figura 4.1. Removemos seus vértices, pois não consideramos órbitas que atingem um dos vértices. Esta construção pode ser visualizada como a sobreposição de duas cópias do triângulo costuradas nas bordas, como uma fronha, e inflada para se tornar tridimensional, excluindo os vértices. Chamaremos esta construção de $\mathcal{D}(T)$. Topologicamente, $\mathcal{D}(T)$ é uma esfera com 3 pontos removidos.

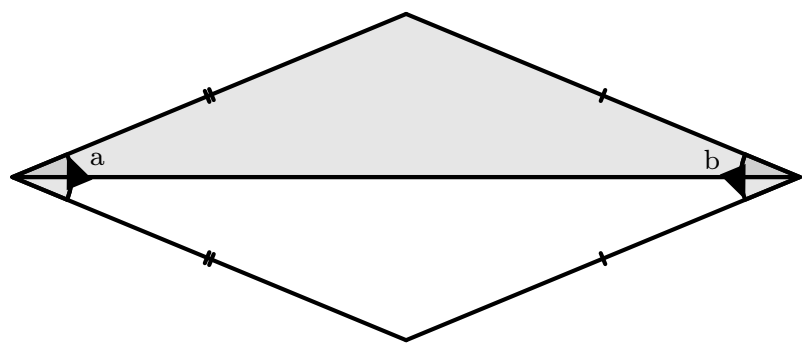

Figura 4.1: $\mathcal{D}(T)$ é a superfície euclidiana formada ao refletir $T$ pelo maior lado, colar os lados pareados e remover os vértices. O resultado é uma esfera com três furos.

A função $f: \mathcal{D}(T) \rightarrow T$ "dobra" $\mathcal{D}(T)$ de volta para formar o triângulo $T$. Se $\gamma$ é uma geodésica fechada ${ }^{1}$ em $\mathcal{D}(T)$, então $f(\gamma)$ é uma órbita bilhar periódica em $T$. Reciprocamente, se $\hat{\gamma}$ é uma órbita bilhar periódica em $T$, existe um levantamento $\gamma$ de $\hat{\gamma}$ tal que $\gamma$ é uma geodésica fechada em $\mathcal{D}(T)$ e $f(\gamma)=\hat{\gamma}$. Veja o diagrama abaixo.

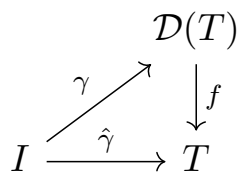

O espaço $\mathcal{D}(T)$ nos permite definir holonomia, que é uma função hol $: \pi_{1}(\mathcal{D}(T)) \rightarrow I_{s o m}\left(\mathbb{R}^{2}\right)$ que a cada elemento $c$ do grupo fundamental de $\mathcal{D}(T)$ associa uma isometria hol $(c)$ do plano que preserva orientação. A noção de holonomia é definida mais geralmente na seção 3.4 de [Thu14] para variedades geométricas. Aqui trataremos dela para o caso particular de $\mathcal{D}(T)$.

Esta função é definida do seguinte modo: existe um isomorfismo canônico $\psi$ entre $\pi_{1}(\mathcal{D}(T))$ e o

\footnotetext{
${ }^{1}$ Lembrando que, se $M$ é uma superfície em $\mathbb{R}^{3}$, uma geodésica de $M$ é uma curva $\alpha$ em $M$ cuja aceleração $\alpha^{\prime \prime}$ é sempre normal a $M$. Uma geodésica fechada é uma geodésica que é uma curva fechada. Intuitivamente, sem aceleração uma geodésica é uma espécie de "linha reta" sobre a superfície.
} 
grupo $G(\widetilde{\mathcal{D}(T)}, \mathcal{D}(T))$ de automorfismos de recobrimento de $\widetilde{\mathcal{D}(T)^{2}}$. Então para cada $c \in \pi_{1}(\mathcal{D}(T))$ associamos o automorfismo $\psi(c): \widetilde{\mathcal{D}(T)} \rightarrow \widetilde{\mathcal{D}(T)}$.

Além disso, dado um aberto $\tilde{U}$ no recobrimento universal $\widetilde{\mathcal{D}(T)}, U=p(\tilde{U})$ é um aberto em $\mathcal{D}(T)$. Como $\mathcal{D}(T)$ é localmente isométrico a $\mathbb{R}^{2}, U$ pode ser imerso isometricamente em $\mathbb{R}^{2}$. Esta imersão define a função desenvolvedora ${ }^{3}$ dev $: \widetilde{\mathcal{D}(T)} \rightarrow \mathbb{R}^{2}$.

Com isso, $h o l(c)$ é definida como a função de $\mathbb{R}^{2}$ em $\mathbb{R}^{2}$ tal que o diagrama a seguir comuta.

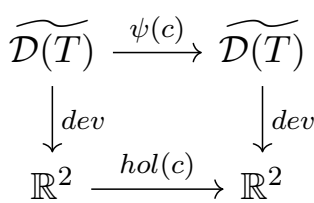

Esta definição de $h o l(c)$ depende da escolha de um ponto base do recobrimento universal e de uma isometria entra uma vizinhança deste ponto e $\mathbb{R}^{2}$, porém o resultado hol $(c)$ dependente destas escolhas será invariante por conjugação em $\operatorname{Isom}_{+}\left(\mathbb{R}^{2}\right)$. As propriedades que consideraremos no grupo fundamental de $\mathcal{D}(T)$ serão invariantes por conjugação, então não é necessário explicitar estas escolhas.

Se $\gamma$ é uma geodésica fechada em $\mathcal{D}(T)$, então seu levantamento $\tilde{\gamma}$ para o recobrimento universal é uma geodésica tal que $\operatorname{dev}(\tilde{\gamma})$ é uma reta. Logo, $h o l(\gamma)$ é uma translação.

O grupo fundamental $\pi_{1}(\mathcal{D}(T))$ é um grupo livre de 2 elementos. Este grupo pode ser "visualizado" como um grupo não abeliano gerado por todas as sequências finitas de símbolos $a, b, a^{-1} \mathrm{e}$ $b^{-1}$. O grupo de homologia $H_{1}(\mathcal{D}(T))^{4}$ é a abelianização de $\pi_{1}(\mathcal{D}(T))$ e, do mesmo modo, pode ser "visualizado" como um grupo abeliano gerado por todas as sequências finitas de $a, b, a^{-1}$ e $b^{-1}$, ou seja, agrupando adequadamente os símbolos, cada elemento do grupo é identificado por um número inteiro para o expoente de $a$ e outro número inteiro para o expoente de $b .^{5}$ Assim, $H_{1}(\mathcal{D}(T))$ é isomorfo a $\mathbb{Z}^{2}$. Veja o apêndice para a abelianização do grupo fundamental para espaços conexos por caminhos.

Com isso, temos a seguinte condição homológica para estabilidade de uma órbita bilhar periódica:

Lema 7. Se uma órbita bilhar periódica $\hat{\gamma}$ em um triângulo $T$ é estável, então $\gamma$ é homóloga a zero em $\mathcal{D}(T)$.

Demonstração. Temos o seguinte diagrama comutativo, no qual as setas para baixo são abelianizações de grupos.

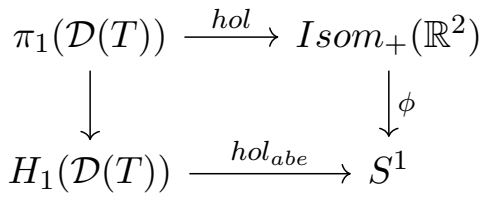

Vejamos que $S^{1}$ é de fato a abelianização de $\operatorname{Isom}_{+}\left(\mathbb{R}^{2}\right)$. Lembramos que a abelianização de um grupo é o quociente deste sobre o subgrupo de seus comutadores $[g, h]=g^{-1} h^{-1} g h$. Dadas $g$ e $h$ duas isometrias que preservam orientação de $\mathbb{R}^{2}$, estas isometrias são da forma $g(x)=M x+a$ e $g(x)=N x+b$ onde $M$ e $N$ são matrizes de rotação $2 \times 2$ e $a, b \in \mathbb{R}^{2}$. Então um comutador de $I_{s o m}\left(\mathbb{R}^{2}\right)$ é da forma $g^{-1} h^{-1} g h=M^{-1} N^{-1} M N x+M^{-1} N^{-1} M b+M^{-1} N^{-1}(a-b)+M^{-1} a$.

\footnotetext{
${ }^{2}$ Ver apêndice.

${ }^{3}$ Do inglês, developing map, definida por William P. Thurston em [Thu14] para variedades geométricas como uma função que, intuitivamente, "desenrola" a variedade usando seu recobrimento universal.

${ }^{4} \mathrm{Em}$ todos os casos neste trabalho o grupo de homologia será sobre o anel $\mathbb{Z}$ dos inteiros, então reduzimos a notação de $H_{1}(X, \mathbb{Z})$ para $H_{1}(X)$.

${ }^{5}$ Esta ideia é um caso da teoria de presentação de um grupo (em inglês, group presentation), muitas vezes chamada em português de "apresentação de um grupo". Todo grupo é isomorfo ao quociente de um grupo livre por um subgrupo normal gerado por relações. Estas relações dizem como os elementos comutam. Veja [MKS66].
} 
Como $M$ e $N$ comutam, temos $g^{-1} h^{-1} g h=x+c$, onde $c=N^{-1} b+M^{-1} N^{-1}(a-b)+M^{-1} a$. Logo, o subgrupo dos comutadores de $I s o m_{+}\left(\mathbb{R}^{2}\right)$ é o grupo de translações. Tomando o quociente $(g \tilde{h}$ se e somente se $g-h$ é uma translação, isto é, $M=N)$, obtemos o grupo de rotações em $R^{2}$, que é isomorfo a $S^{1}$.

Assim, se $\hat{\gamma}$ é uma órbita bilhar periódica, $h o l(\gamma)$ é uma translação e $\phi(h o l(\gamma))=0$.

Se, além disso, $\hat{\gamma}$ for estável, então existe um triângulo $T^{\prime}$ próximo de $T$ tal que os ângulos de $T^{\prime}$ são racionalmente independentes, isto é, se $n_{1} \alpha+n_{2} \beta+n_{3}(\pi-\alpha-\beta)=0 \operatorname{com} n_{1}, n_{2}, n_{3} \in \mathbb{Z}$ então $n_{1}=n_{2}=n_{3}=0$.

O primeiro grupo de homologia de $\mathcal{D}\left(T^{\prime}\right)$ é gerado por dois laços $a$ e $b$ indicados na figura 4.1. $h_{a b}(a)$ e $h o l_{a b e}(b)$ são rotações com ângulos $2 \alpha$ e $2 \beta$, respectivamente. Como $\alpha, \beta$ e $\pi$ são racionalmente independentes, então hol abe $: H_{1}(\mathcal{D}(T)) \rightarrow S^{1}$ é injetora. Como $h o l\left(\gamma^{\prime}\right)$ é uma translação, então $\phi\left(h o l\left(\gamma^{\prime}\right)\right)=0$ e, pela injetividade de $h o l_{a b e}, \gamma^{\prime}$ deve ser homóloga a 0 .

Como $\hat{\gamma}^{\prime}$ e $\hat{\gamma}$ percorrem os mesmos lados dos triângulos $T^{\prime}$ e $T$, então $\gamma$ também é homóloga a 0 .

À luz do teorema 2.0.1, podemos pensar no lema 7 do seguinte modo: como o grupo de homologia de $\mathcal{D}(T)$ é gerado pelos dois laços $a$ e $b$, consideramos $-a$ e $-b$ estes laços percorridos no sentido contrário. Uma órbita periódica $\hat{\gamma}$ é estável se e somente se a palavra que a representa é estável. Mas isto é equivalente ao seu levantamento $\gamma$ pela $f$ percorrer os laços $a$ e $-a$ o mesmo número de vezes e o mesmo para os laços $b$ e $-b$, que, por sua vez, é equivalente a $\gamma$ ser homóloga a 0 . Portanto, a recíproca do lema 7 também é verdadeira.

\section{1 Órbitas bilhares periódicas em triângulos retângulos são instá- veis}

Nesta seção, usaremos o lema 7 para mostrar o seguinte resultado.

Teorema 4.1.1. Toda órbita bilhar periódica em um triângulo retângulo é instável.

Seja $G(T)$ o grupo de rotações de $T$, isto é, o grupo de isometrias lineares do plano gerado por reflexões nos lados do triângulo $T$. A superfície de translação $S(T)$ é a união disjunta de triângulos $g(T)$, com $g \in G(T)$ com as seguintes identificações:

1. Dois triângulos $g_{1}(T)$ e $g_{2}(T)$ são identificados por uma translação se $g_{1} \circ g_{2}^{-1}$ é uma translação, isto é, eles são paralelos e tem a mesma orientação;

2. O $i$-ésimo lado dos triângulos $g_{1}(T)$ e $g_{2}(T)$ é identificado por uma translação se $g_{1} \circ g_{2}^{-1}$ pode ser escrito como a composição de uma reflexão e uma translação, isto é, uma reflexão no lado $i$ de $g_{1}(T)$ gera um triângulo paralelo a $g_{2}(T)$.

A superfície resultante $S(T)$ é a menor superfície de translação que é um espaço de recobrimento para $\mathcal{D}(T)$. A função de recobrimento $p: S(T) \rightarrow \mathcal{D}(T)$ leva cada triângulo de $S(T)$ no triângulo de $\mathcal{D}(T)$ que tem a mesma orientação.

Os casos mais simples de superfícies de translação são para triângulos racionais, e estas superfícies são minuciosamente estudadas em [MT02], especialmente as superfícies de Veech, que são as superfícies de translação para triângulos de Veech. Veremos melhor este caso na seção 4.2. Este é o caso mais simples pois, se $T$ é racional, $G(P)$ é finito e temos finitos triângulos formando $S(T)$. Se $T$ é irracional, $G(P)$ é infinito e $S(T)$, com infinitos triângulos, se torna mais complexa. Para o caso em que $T$ é retângulo, felizmente, podemos obter $S(T)$ de forma simplificada pois as reflexões de $T$ geram losangos, e losangos tem lados opostos paralelos.

Note que basta mostrar o teorema 4.1.1 para triângulos irracionais. De fato, se toda órbita bilhar periódica em um triângulo retângulo irracional é instável, pela densidade desta classe de triângulos retângulos e pelo fato de todo ladrilho de órbita estável ser aberto, não pode existir órbita bilhar periódica estável em nenhum triângulo retângulo. 
No caso irracional e retângulo, $S(T)$ é a união infinita de losangos $R_{k}$ com $k \in \mathbb{Z}$, onde cada losango $R_{k}$ tem dois lados opostos colados no losango $R_{k-1}$ e dois lados opostos colados no losango $R_{k+1}$. Veja a figura 4.2 .

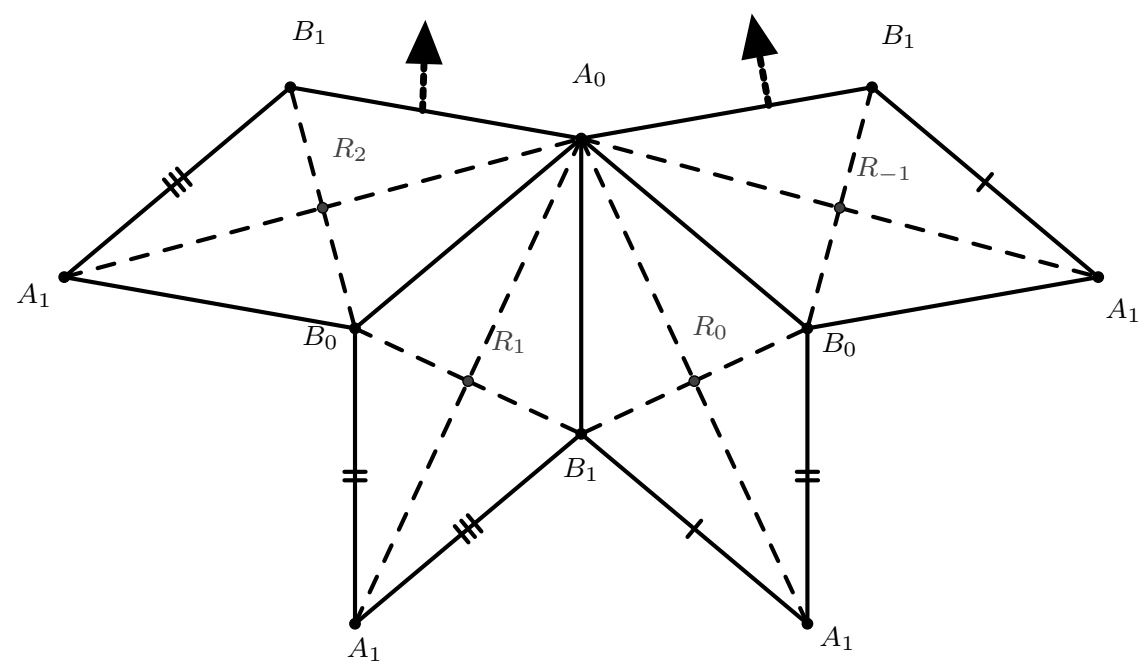

Figura 4.2: Superfície de translação $S(T)$ para um triângulo irracional $T$.

Os losangos que formam $S(T)$ têm "furos" no seu centro e em seus vértices. Estes furos são o levantamento por $p$ dos "furos" de $\mathcal{D}(T) . S(T)$ tem 4 pontos onde ocorrem ramificações de várias arestas, indicados na figura 4.2 como $A_{0}, A_{1}, B_{0}$ e $B_{1}$.

O grupo de automorfismos $G(S(T) \mid \mathcal{D}(T))$ do recobrimento $p: S(T) \rightarrow \mathcal{D}(T)$ é isomórfico a $\mathbb{Z} \times \mathbb{Z}_{2}$. Logo, a única involução ${ }^{6}$ em $G(S(T) \mid \mathcal{D}(T))$ é a função $i: S(T) \rightarrow S(T)$ que rotaciona com um ângulo $\pi$ em torno no centro de cada losango $R_{k}$. Esta função troca os pontos $A_{0}$ e $A_{1}$ e troca os pontos $B_{0}$ e $B_{1}$. Então $G(S(T) \mid \mathcal{D}(T))$ é formado por transformações que "deslocam" os losangos por um número inteiro $n$ ( $R_{k}$ é levado em $R_{k+n}$ isometricamente) compostas ou não com a involução $i$, isto justifica o fato de ser isomórfico a $\mathbb{Z} \times \mathbb{Z}_{2}$.

Demonstração do teorema 4.1.1. Seja $T$ um triângulo retângulo, $\hat{\gamma}$ uma órbita bilhar periódica em $T$ e $\gamma$ a geodésica fechada correspondente em $\mathcal{D}(T)$. Como $h o l(\gamma)$ é uma translação, o levantamento $\tilde{\gamma}$ de $\gamma$ em $S(T)$ é uma geodésica simples fechada em $S(T)$.

As duas curvas fechadas $\tilde{\gamma}$ e $i(\tilde{\gamma})$ cortam a superfície $S(T)$ em duas partes. Uma dessas partes é um cilindro com dois furos cuja fronteira é formada pelas curvas $\tilde{\gamma}$ e $i(\tilde{\gamma})$. De fato, note que tais curvas são disjuntas e $\tilde{\gamma}$ intercepta arestas opostas de cada losango o mesmo número de vezes, e o mesmo vale para $i(\tilde{\gamma})$. Em cada losango $R_{k}$, colorimos os pedaços de $R_{n} \backslash(\tilde{\gamma} \cup i(\tilde{\gamma}))$ de preto e branco do seguinte modo: colorimos a parte que contém $A_{0}$ de branco e em seguida intercalamos preto e branco para cada pedaço. Veja a figura 4.3.

Como $\tilde{\gamma} \cup i(\tilde{\gamma})$ intercepta cara aresta de $R_{k}$ um número par de vezes, os pedaços com os vértices $B_{0}, B_{1}$ e $A_{1}$ também serão brancos. Isso também implica que esta coloração é compatível com os losangos adjacentes.

Então $S(T) \backslash(\tilde{\gamma} \cup i(\tilde{\gamma}))$ é composto de pelo menos duas componentes, uma branca e uma preta. Vizinhanças dos pontos $A_{0}, A_{1}, B_{0}$ e $B_{1}$ são brancas e $S(T)$ não possui outras ramificações de

\footnotetext{
${ }^{6}$ Uma involução $a$ é um elemento de um grupo $G$ tal que $a^{2}=1$, com 1 o elemento neutro de $G$. Em particular, se $G$ é um grupo de funções, uma involução é uma função que é sua própria inversa.
} 


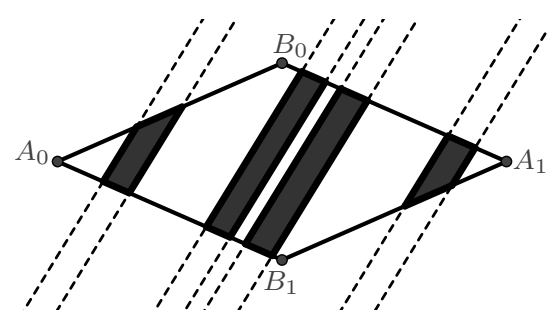

Figura 4.3

arestas. Assim, a parte preta de $S(T) \backslash(\tilde{\gamma} \cup i(\tilde{\gamma}))$ é uma superfície euclidiana orientada delimitada por duas geodésicas, portanto, é um cilindro. Chamaremos $S(T) \backslash(\tilde{\gamma} \cup i(\tilde{\gamma}))$ de $\mathcal{C}$.

Por construção, a involução $i$ preserva $\mathcal{C}$, trocando as componentes da fronteira $\tilde{\gamma}$ e $i(\tilde{\gamma})$. Por isso, $i$ tem exatamente dois pontos fixos em $\mathcal{C}$. Mas como $i$ é um automorfismo de recobrimento não-trivial de $S(T), i$ não tem pontos fixos. Note, no entanto, que os centros de cada losango são pontos fixos de $i$. Portanto, $\mathcal{C}$ tem dois furos que são os centro de dois losangos.

Podemos definir duas curvas $p_{1}$ e $p_{2}$ que contornam cada um dos furos de $\mathcal{C}$ de modo que a união $\tilde{\gamma} \cup i(\tilde{\gamma}) \cup p_{1} \cup p_{2}$ limita uma subsuperfície simplesmente conexa de $S(T)$ e $\llbracket \tilde{\gamma} \rrbracket+\llbracket i(\tilde{\gamma}) \rrbracket+\llbracket p_{1} \rrbracket+\llbracket p_{2} \rrbracket=0$.

Como $i$ é um automorfismo de recobrimento em $S(T)$, a função de recobrimento que leva $S(T)$ em $\mathcal{D}(T)$ leva $\tilde{\gamma}$ e $i(\tilde{\gamma})$ em $\gamma$. Do mesmo modo, esta função leva $p_{1}$ e $p_{2}$ na curva $p$ em $\mathcal{D}(T)$. Esta curva $p$ contorna duas vezes o ponto de $\mathcal{D}(T)$ que é o levantamento do vértice do ângulo $\pi$ (este ponto em $\mathrm{S}(\mathrm{T})$ é o centro de cada losango). Logo, em $H_{1}(\mathcal{D}(T))$

$$
\llbracket \gamma \rrbracket=-\llbracket p \rrbracket \neq 0
$$

e $\gamma$ não é estável.

\subsection{Estabilidade de órbitas bilhares periódicas em triângulos de Veech}

Para demonstrar o teorema 3.0.1, que afirma que é possível cobrir o segmento dos triângulos isósceles no espaço dos parâmetros com ladrilhos de órbitas estáveis, tivemos que dividir em vários casos e provar três teoremas. Todo o capítulo 3 foi dedicado a isso.

O que nós fizemos foi encontrar uma família bi-infinita de palavras $Y_{n, m}$ para cobrir uma vizinhança de todos os pontos que não eram da forma $V_{n}=\left(\frac{\pi}{2 n}, \frac{\pi}{2 n}\right), n=3,4,5, \ldots$ Depois disso, cobrimos os quadrantes $(-,+),(-,-)$ e $(+,-)$ de uma vizinhança de cada ponto $V_{n}$ com os ladrilhos gerados pelas palavras $A_{n}, B_{n}$ e $C_{n}$. E, por fim, precisamos de mais uma família bi-infinita de palavras $W_{n, k}$ para cobrir os quadrantes $(+,+)$ de uma vizinhança de cada $V_{n}$.

Este trabalho todo gira em torno da dificuldade de cobrir uma vizinhança de $V_{n}$ com ladrilhos de órbita. Mais precisamente, veremos a seguir o teorema 3.0.2, isto é, que $V_{2^{k}}=\left(\frac{\pi}{2^{k+1}}, \frac{\pi}{2^{k+1}}\right)$, com $k=3,4,5, \ldots$, não está contido no interior de nenhum ladrilho de órbita e que se $n \geq 3$ não é uma potência de dois, $V_{n}$ está contido em um ladrilho de órbita.

Como para os "pontos problema" $V_{2^{k}}$ teríamos que fazer todo o trabalho de cobrir sua vizinhança com infinitos ladrilhos de órbita, fizemos este trabalho generalizado para todo $V_{n}$, e por isso a demonstração do teorema 3.0.1 é tão longa.

Lembrando, da seção anterior, que a superfície de translação de um polígono é a união disjunta de cópias do polígono obtidas pela ação de seu grupo de simetria com algumas identificações. Quando o polígono é racional, esta superfície é formada por finitas cópias do polígono.

Especificamente no caso dos triângulos de Veech $V_{n}$, começamos com uma cópia de $V_{n}$ no plano com seu maior lado no eixo $x$. Depois refletimos metade deste triângulo ao redor de um dos ângulos agudos e outra metade ao redor do outro. Como os ângulos agudos medem $\pi / 2 n$ e essas metades são triângulos retângulos, cada metade resultará em um polígono regular de $2 n$ lados. Veja a figura 
4.4 que ilustra esta construção e mostra as identificações por translação.

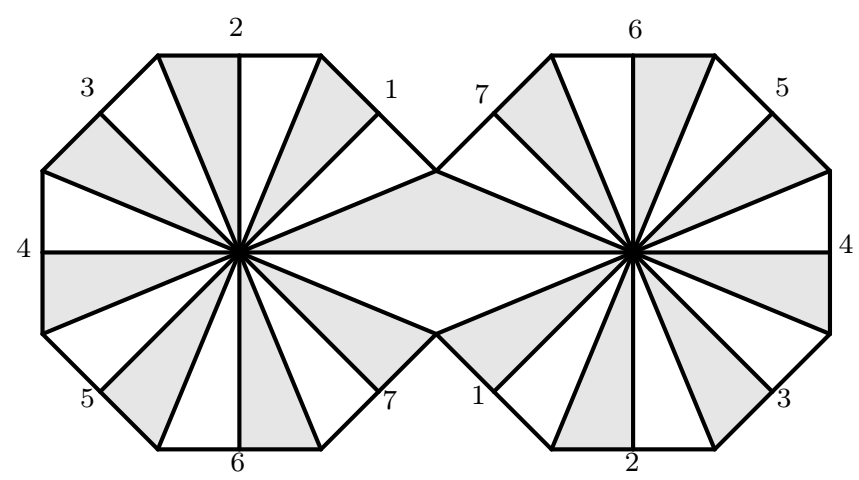

Figura 4.4: Superfície de translação $S\left(V_{4}\right)$. Os triângulos de mesma cor tem a mesma orientação e os números indicam identificação dos lados.

Precisamos de dois polígonos de $2 n$ lados para formar $S\left(V_{n}\right)$ pois em apenas um polígono não podemos identificar os lados opostos devido à orientação dos triângulos.

Assim como na construção de $D(T)$ no início do capítulo, removemos os vértices do triângulo antes de formar a superfície $S\left(V_{n}\right)$, já que não consideramos órbitas que atingem vértices. Com isso, a superfície $S\left(V_{n}\right)$ possui $2 n+2$ furos, dois no centro dos polígonos e $2 n$ em seus vértices.

Uma superfície de translação herda do plano a noção de direção. No plano, essa direção em um ponto é um elemento do fibrado tangente unitário, e o vetor tangente é representado pelo ângulo que ele faz com a horizontal.

Seja $S L(2, \mathbb{R})$ o grupo das matrizes $2 \times 2$ reais com determinante 1 . Definimos uma ação de $S L(2, \mathbb{R})$ no conjunto das superfícies de transalação do seguinte modo: para cada $A \in S L(2, \mathbb{R})$ associamos uma transformação que leva uma superfície de translação em outra. Seja $S$ uma superfície de translação formada pela família $\left\{P_{i}\right\}_{i \in \Lambda}$ de polígonos com suas identificações por translação. Então $A(S)$ é a superfície de translação formada pela família de polígonos $\left\{A\left(P_{i}\right)\right\}_{i \in \Lambda}$. As identificações por translação são preservadas por $A$, que preserva linhas paralelas e a proporção entre elas. Por abuso de linguagem, chamaremos de $A$ a restrição aos polígonos $P_{i}$ da ação de $A$ no plano.

Definição 4.2.1. O grupo de Veech de $\mathrm{S}$, denotado por $\Gamma(S)$, é o subgrupo de $S L(2, \mathbb{R})$ formado pelas $A$ tais que existe uma isometria que preserva direção $\varphi_{A}: A(S) \rightarrow S$.

A função $\varphi_{A} \circ A: S \rightarrow S$ é um automorfismo afim de $S$ e o grupo formado pelas $\varphi_{A} \circ A$ com $A \in \Gamma(S)$ é chamado de grupo de automorfismos afins.

O grupo de Veech é sempre um grupo discreto (no sentido topológico).

Definição 4.2.2. Uma superfície de Veech é uma superfície de translação cujo grupo de Veech é um reticulado em $S L(2, \mathbb{R})$.

Em uma superfície de Veech, as geodésicas fechadas tem comportamento conhecido. Mais precisamente, dizemos que uma direção $\theta \in \mathbb{R} / 2 \pi \mathbb{Z}$ é completamente periódica se toda geodésica biinfinita nesta direção é fechada. Temos o seguinte resultado de Veech.

Teorema 4.2.1 (Dicotomia de Veech). Seja $S$ uma superfície de Veech e $\theta \in \mathbb{R} / 2 \pi \mathbb{Z}$ uma direção. Então $\theta$ é completamente periódica em $S$ ou o fluxo geodésico na direção $\theta$ é unicamente ergódico. Além disso, $\theta$ é completamente periódica se e somente se existe uma matriz parabólica ${ }^{7} A \in \Gamma(S)$ para a qual $\theta$ é um autovetor.

Para mais detalhes em grupos de Veech, veja a seção 5 de [MT02]. Vamos precisar do seguinte resultado, que é uma consequência do trabalho de Veech e pode ser visto na seção 9 de [HS09].

\footnotetext{
${ }^{7} A \in S L(2, \mathbb{R})$ é parabólica se possui apenas um autovalor, e este deve ser 1 ou -1 .
} 
Lema 8. Se $p_{1}$ é uma geodésica fechada em $S\left(V_{n}\right)$, então existe uma geodésica fechada $p_{0}$ nas direçôes 0 ou $\frac{\pi}{2 n}$ e um automorfismo afim $\varphi_{D} \circ D$ que leva $p_{0}$ em $p_{1}$.

Com isso, vamos estabelecer um critério para estabilidade de uma órbita bilhar periódica em $V_{n}$ usando o grupo de automorfismos afins. Faremos isso usando homologia, como na seção anterior.

Lembre-se que $H_{1}\left(\mathcal{D}\left(V_{n}\right)\right) \cong \mathbb{Z}^{2}$ e que escolhemos seus geradores como as curvas $a$ e $b$ que contornam os vértices agudos, como na figura 4.1. Do mesmo modo, $S\left(V_{n}\right)$ tem $2 n+2$ furos e $H_{1}\left(S\left(V_{n}\right)\right) \cong \mathbb{Z}^{2 n+1}$. Vamos escolher seus geradores como $\mathcal{B}=\left\{\beta_{1}, \beta_{-1}, \lambda_{1-n}, \ldots, \lambda_{n+1}\right\}$, ilustrados na figura 4.5 .

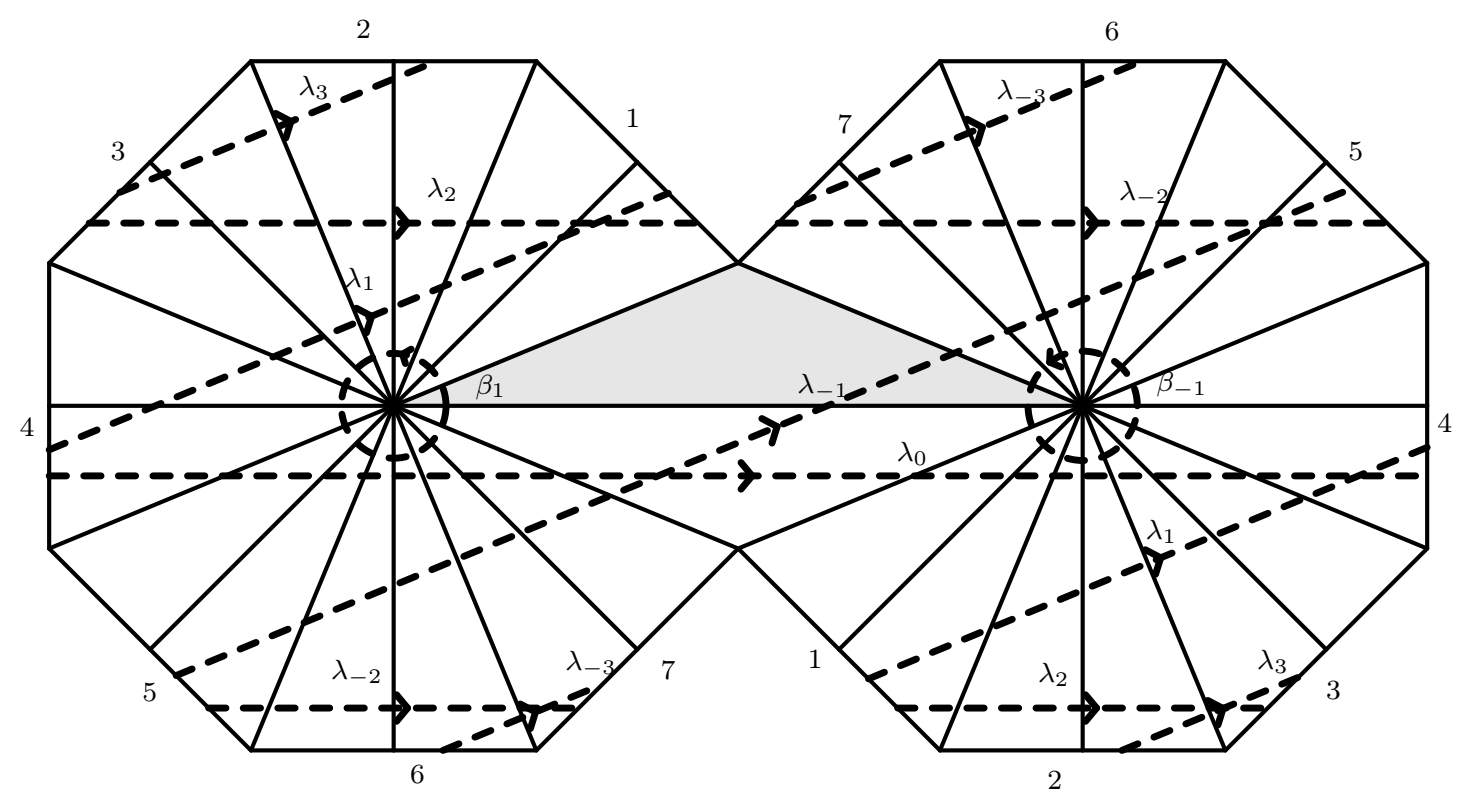

Figura 4.5: Superfície de translação $S\left(V_{4}\right)$ com elementos do grupo de homologia destacados.

As classes de homologia $\lambda_{k}$ foram nomeadas do seguinte modo: se $k$ é par, sua inclinação é 0 e se $k$ é ímpar, sua inclinação é $\frac{\pi}{2 n}$. O índice $k$ cresce de baixo pra cima no polígono da esquerda. Com essa nomenclatura, $\lambda_{2 i}$ intercepta $\lambda_{2 i+1}$ ou $\lambda_{2 i-1}$ em um único ponto e outras curvas não tem interseção.

Seja $H^{1}\left(S\left(V_{n}\right)\right)$ o primeiro grupo de cohomologia de $S\left(V_{n}\right)^{8}$. Denotamos por $\mathcal{B}^{*}=$ $\left\{\beta_{1}^{*}, \beta_{-1}^{*}, \lambda_{1-n}^{*}, \ldots, \lambda_{n+1}^{*}\right\}$ a base de $H^{1}\left(S\left(V_{n}\right)\right)$.

Se $\phi: S\left(V_{n}\right) \rightarrow \mathcal{D}\left(V_{n}\right)$ é a função de recobrimento que leva cada triângulo de $S\left(V_{n}\right)$ no triângulo de $\mathcal{D}\left(V_{n}\right)$ com a mesma orientação, então $\phi$ induz uma função de $H_{1}\left(S\left(V_{n}\right)\right)$ para $H_{1}\left(\mathcal{D}\left(V_{n}\right)\right)$ que satisfaz

1. $\phi\left(\beta_{1}\right)=2 n a ;$

2. $\phi\left(\beta_{-1}\right)=2 n b$;

3. $\phi\left(\lambda_{k}\right)= \begin{cases}(n+k) a-(n+k) b & \text { se } k<0, \\ n a+n b & \text { se } k=0, \\ (n-k) a+(n-k) b & \text { se } k>0 .\end{cases}$

Usamos $\phi$ para escrever os elementos de $H_{1}\left(S\left(V_{n}\right)\right)$ em função de $a$ e $b$. Podemos escrever os elementos do grupo de cohomologia $H^{1}\left(S\left(V_{n}\right)\right)$ usando os coeficientes de $a$ e $b$. Sejam $\phi_{1}^{*}$ e $\phi_{-1}^{*}$ esses elementos. Como eles são elementos do dual de $H_{1}\left(S\left(V_{n}\right)\right), \phi_{1}^{*}, \phi_{-1}^{*}: H_{1}\left(S\left(V_{n}\right)\right) \rightarrow \mathbb{Z}$. Eles satisfazem $\phi(x)=\phi_{1}^{*}(x) a+\phi_{-1}^{*}(x) b$ e temos

\footnotetext{
${ }^{8}$ Note que a diferença de notação é na posição do índice 1. $H_{1}$ é para homologia e $H^{1}$ para cohomologia. Podemos pensar em cohomologia como o dual de homologia.
} 


$$
\begin{gathered}
\phi_{1}^{*}=2 n \beta_{1}^{*}+\lambda_{1-n}^{*}+2 \lambda_{2-n}^{*}+\cdots+n \lambda_{0}^{*}-(n-1) \lambda_{1}^{*}-\cdots-\lambda_{n-1}^{*}, \\
\phi_{-1}^{*}=2 n \beta_{-1}^{*}-\lambda_{1-n}^{*}-2 \lambda_{2-n}^{*}-\cdots-n \lambda_{0}^{*}+(n-1) \lambda_{1}^{*}+\cdots+\lambda_{n-1}^{*} .
\end{gathered}
$$

Se $g$ é um elemento do grupo de automorfismos afins de $S\left(V_{n}\right)$, denotamos por $g$ a função induzida $g: H_{1}\left(S\left(V_{n}\right)\right) \rightarrow H_{1}\left(S\left(V_{n}\right)\right)$ e por $g^{*}$ o pullback para a cohomologia $g^{*}: H^{1}\left(S\left(V_{n}\right)\right) \rightarrow$ $H^{1}\left(S\left(V_{n}\right)\right)$. Isto é, se $\eta^{*} \in H^{1}\left(S\left(V_{n}\right)\right)$, então $g^{*}\left(\eta^{*}\right):=\eta^{*} \circ g^{-1}$.

O grupo de automorfismos afins de $S\left(V_{n}\right)$ é gerado por $\sigma, \tau_{o}$ e $\tau_{e}$. $\sigma$ é a involução que troca os dois polígonos de $S\left(V_{n}\right), \tau_{o}$ é a torção de Dehn à direita ${ }^{9}$ na direção dos cilindros gerados pelas $\lambda_{k} \operatorname{com} k$ ímpar e $\tau_{e}$ é a torção de Dehn à direita na direção dos cilindros gerados pelas $\lambda_{k} \operatorname{com} k$ múltiplo de 2 .

Este fato é consequência do seguinte teorema, também de Veech, cuja demonstração pode ser consultada em [MT02].

Teorema 4.2.2. O grupo $\Gamma\left(S\left(V_{n}\right)\right)$ é gerado por matrizes parabólicas que fixam as direções 0 e $\frac{\pi}{2 n}$. O grupo de automorfismos afins de $S\left(V_{n}\right)$ age como uma torção de Dehn à direita em cada cilindro maximal na direção de seus autovetores.

Com isso, podemos enunciar o lema de estabilidade. As funções induzidas por $\sigma, \tau_{o}$ e $\tau_{e}$ e seus respectivos pullbacks são usadas para demonstrá-lo.

Lema 9 (Estabilidade). Seja $p$ uma órbita bilhar periódica em $V_{n}$, $\tilde{p}$ seu levantamento para $S\left(V_{n}\right)$ e $x$ a classe de homologia de $\tilde{p}$. Pelo lema $8, x=w\left(\lambda_{k}\right)$ para algum automorfismo afim $w$ no grupo $\left\langle\sigma, \tau_{o}, \tau_{e}\right\rangle$ e algum $k \in\{1-n, \ldots, n-1\}$. Então $p$ é estável se, e somente se, $k \neq 0$ e, escrevendo $\left(w^{*}\right)^{-1}\left(\phi_{1}^{*}\right)$ na base $\mathcal{B}^{*}$, o coeficiente de $\lambda_{k}^{*}$ é 0 .

Com este lema, Hooper e Schwartz mostram que não existe órbita estável em $V_{2^{m}}$ com $m=$ $\{2,3,4, \ldots\}$ e, para o caso $V_{n}$ com $n$ natural maior que 3 que não é uma potência de 2 , eles exibem classes de homologia de geodésicas em $S\left(V_{n}\right)$ que projetam para órbitas estáveis.

\footnotetext{
${ }^{9}$ Uma torção de Dehn à direita (esquerda) em uma curva fechada simples $\gamma$ em uma superfície é um homeomorfismo que fixa $\gamma$ e torce qualquer outra curva $\nu$ que intercepta $\gamma$ uma vez à direita (esquerda) de $\gamma$.
} 


\section{Apêndice A}

\section{Topologia algébrica}

A topologia algébrica é a área da matemática que surgiu com a busca por estruturas algébricas associadas a objetos topológicos que são invariantes por "deformação" ou homeomorfismo, permitindo assim caracterizar estes objetos através das estruturas algébricas associadas a elas.

As "lentes" da topologia algébrica que permitem ver estes espaços através das suas estruturas algébricas são formalmente conhecidas como funtores $^{1}$, e estes levem funções contínuas entre espaços topológicos em homomorfismos de grupos, como veremos com os homomorfismos induzidos.

A seguir, definiremos alguns conceitos que serão utilizados no capítulo 4 . O leito interessado pode consultar [Bre93, Hat02, Mun00, Mun84]. Para referências em português, temos [Lim06, Lim12].

Por convenção, $I$ sempre será o intervalo real $[0,1]$ e todas as funções serão contínuas a menos de menção contrária.

\section{A.1 Homotopia}

Intuitivamente, uma homotopia é uma transformação que deforma funções continuamente. Duas funções são homotópicas se existe uma deformação contínua que leva uma na outra. Mais formalmente, definimos:

Definição A.1.1. Dados dois espaços topológicos $X$ e $Y$, uma homotopia de funções de $X$ para $Y$ é uma função $F: X \times I \rightarrow Y$. Outra maneira de pensar numa homotopia é como uma família de funçôes $F_{t}: X \rightarrow Y$ tais que $t \in I$ e $F_{t}$ depende continuamente de $t$.

Duas funções $f: X \rightarrow Y$ e $g: X \rightarrow Y$ são homotópicas, e denotamos por $f \simeq g$, se existe uma homotopia $F: X \times I \rightarrow Y$ tal que $F(x, 0)=f(x)$ e $F(x, 1)=g(x)$

Vamos agora trabalhar com algumas propriedades de homotopia que serão usadas para falar de grupo fundamental, porém as enunciaremos agora com mais generalidade para dar uma luz ao conceito de grupo de homotopia.

A relação $f \simeq g$ de homotopia entre funções é uma relação de equivalência. Lembre que para ser uma relação de equivalência, a relação de homotopia deve satisfazer as seguintes propriedades, para $f, g, h: X \rightarrow Y$ :

Refrexiva $f \simeq f$;

Simétrica $f \simeq g \Longrightarrow g \simeq f$

Transitiva $f \simeq g$ e $g \simeq h \Longrightarrow f \simeq h$.

A propriedade reflexiva é satisfeita tomando a homotopia $F(x, t)=f(x)$ para todo $t \in I$. A propriedade simétrica é satisfeita pois, dada uma homotopia $F: X \times I \rightarrow Y \operatorname{com} F(x, 0)=f(x)$ e

\footnotetext{
${ }^{1}$ Veja a página 162 de [Hat02] para mais detalhes de homologias como funtores. Recomendamos aos leitores curiosos [ML13] para mais detalhes em teoria das categorias.
} 
$F(x, 1)=g(x)$, podemos definir uma homotopia $F^{-1}(x, t)=F(x, 1-t)$ que percorre a variável $t$ "de trás pra frente".

Para a transitividade, seja $F$ a homotopia que leva $f$ em $g$ e $G$ a homotopia que leva $g$ em $h$. Defino a homotopia F.G, que é a concatenação de $F$ e $G$ por

$$
F . G(x, t)= \begin{cases}F(x, 2 t) & \text { se } t \in\left[0, \frac{1}{2}\right] \\ G(x, 2 t-1) & \text { se } t \in\left[\frac{1}{2}, 1\right]\end{cases}
$$

Como esperado, se podemos deformar $f$ em $g$ e deformar $g$ em $h$, se "dobrarmos a velocidade" destas deformações, podemos deformar $f$ em $h$ no mesmo intervalo de tempo unitário. Claro que não precisamos combinar as duas homotopias no ponto $t=\frac{1}{2}$, isso poderia ser feito em qualquer outro ponto com outra "velocidade".

Vejamos agora algumas propriedades que sugerem a possibilidade de definir uma operação de grupos a partir da concatenação de duas homotopias.

Para que possamos concatenar duas homotopias, uma delas deve terminar onde a outra começa. Dada esta ressalva, quando fazemos o quociente de todas as homotopias pela relação de equivalência $\simeq$ e definimos uma operação entre os elementos deste quociente, temos um grupo. Para mais detalhes de como isto é feito para homotopias entre espaços quaisquer, veja o capítulo 3 de [Bre93].

Definição A.1.2. Se $A \subset X$ então uma homotopia $F: X \times I \rightarrow Y$ é relativa a A, e denotamos por rel A se $F(a, t)$ não depende de $t$ para todo $a \in A$.

Uma homotopia rel $X$ é constante. Se pensarmos em homotopias entre homotopias, uma homotopia rel $X \times \partial I=X \times\{0,1\}$ relaciona homotopias que começam e terminam no mesmo ponto. Esta ideia está presente no seguinte lema.

Lema 10 (Reparametrização). Seja $F: X \times I \rightarrow Y$ uma homotopia e $\phi_{1}, \phi_{2}:(I, \partial I) \rightarrow(I, \partial I)$ funções cujos valores em $\partial I$ coincidem. Definimos uma reparametrização de $F$ por $\phi_{i}$ por $G_{i}(x, t)=$ $F\left(x, \phi_{i}(t)\right)$ para $i=1,2$. Então $G_{1} \simeq G_{2}$ rel $X \times \partial I$.

Demonstração. Definimos $H: X \times I \times I \rightarrow Y$ por $H(x, t, s)=F\left(x, s \phi_{2}(t)+(1-s) \phi_{1}(t)\right)$. Logo

$$
\begin{aligned}
& H(x, t, 0)=F\left(x, \phi_{1}(t)\right)=G_{1}(x, t), \\
& H(x, t, 1)=F\left(x, \phi_{2}(t)\right)=G_{2}(x, t),
\end{aligned}
$$

isto é, $H$ é uma homotopia entre $G_{1}$ e $G_{2}$ e

$$
\begin{aligned}
& H(x, 0, s)=F\left(x, s \phi_{2}(0)+(1-s) \phi_{1}(0)\right)=F\left(x, s \phi_{1}(0)\right)=G_{1}(x, 0), \\
& H(x, 1, s)=F\left(x, s \phi_{2}(1)+(1-s) \phi_{1}(1)\right)=F\left(x, s \phi_{2}(1)\right)=G_{2}(x, 1),
\end{aligned}
$$

que não dependem de $s$.

Denotamos por $C$ a homotopia constante. Se $F: X \times I \rightarrow Y$ é uma homotopia, F.C é a concatenação com $C(x, t)=F(x, 1)$ (depois de chegar em $F(x, 1)$, a homotopia $F . C$ continua constante) e $C . F$ é a concatenação com $C(x, t)=F(x, 0)(C . F$ é constante até chegar em $F(x, 0)$ ).

Sejam $F, G, H, J: X \times I \rightarrow Y$ homotopias. Usando o lema de reparametrização, mostramos as seguintes propriedades

1. $F . C \simeq F \operatorname{rel} X \times \partial I$ e $C . F \simeq F$ rel $X \times \partial I$;

2. $F . F^{-1} \simeq C \operatorname{rel} X \times \partial I$;

3. $(F . G) . H \simeq F .(G . H)$ rel $X \times \partial I$;

4. Se $F \simeq G \operatorname{rel} X \times \partial I$ e $H \simeq J \operatorname{rel} X \times \partial I$, então $F . H \simeq G$. $J$ rel $X \times \partial I$. 
As propriedades 1,2 e 3 implicam que a concatenação define uma operação de grupos em homotopias homotópicas rel $X \times \partial I$ e a propriedade 4 implica que este produto está bem definido módulo homotopia (por isso tomamos o quociente).

No próximo capítulo veremos o primeiro e mais simples grupo de homotopia, o grupo fundamental.

\section{A.2 Grupo fundamental}

Neste capítulo, definiremos o grupo fundamental de um espaço topológico, que é um caso especial de grupo de homotopia. Para isso, trataremos de um caso especial de homotopia, a homotopia de caminhos definida a seguir.

Definição A.2.1. Um caminho em $X$ é uma função $f: I \rightarrow X$. Uma homotopia de caminhos em $X$ é uma família $f_{t}: I \rightarrow X, 0 \leq t \leq 1$, tal que

1. $f_{t}(0)=x_{0}$ e $f_{t}(1)=x_{1}$ são independentes de $t$, isto é, para todo $t \in I$ o caminho $f_{t}$ começa em $x_{0}$ e termina em $x_{1}$.

2. $F: I \times I \rightarrow X$ definido por $F(s, t)=f_{t}(s)$ é contínua.

Dizemos que $f_{0}$ e $f_{1}$ são caminhos homotópicos e denotamos por $f_{0} \simeq f_{1}$

Ou seja, uma homotopia de caminhos é uma homotopia de funções de $I$ para $X$. Poderíamos ter usado a notação $F(s, t)$ da seção anterior, porém a notação $f_{t}(s)$ ajuda a distinguir o parâmetro $s$ de cada caminho do parâmetro $t$ da família. Naturalmente, todas as propriedades de homotopias listadas na seção anterior se aplicam para homotopias de caminhos, e o grupo de homotopias definido por homotopias de caminhos são de especial interesse.

$\mathrm{Na}$ seção anterior, fizemos a concatenação (no parâmetro $t$ ) de homotopias. Agora podemos falar da concatenação (no parâmetro $s$ ) de caminhos.

Definição A.2.2. Dados dois caminhos $f, g: I \rightarrow X$ tais que $f(1)=f(0)$, existe uma composição ou produto $f . g$ definida por

$$
f . g(s)= \begin{cases}f(2 s) & \text { se } s \in\left[0, \frac{1}{2}\right] \\ g(2 s-1) & \text { se } s \in\left[\frac{1}{2}, 1\right]\end{cases}
$$

Definição A.2.3. Considere em um espaço topológico $X$ caminhos que começam e terminam no mesmo ponto $f(0)=f(1)=x_{0}$. Chamamos estes caminhos de laços $e x_{0}$ de ponto base. $O$ grupo fundamental de $X$ no ponto base $x_{0}$, denotado por $\pi_{1}\left(X, x_{0}\right)$, é o grupo formado por todas as classes de homotopia $[f]$ de laços $f: I \rightarrow X$ no ponto base $x_{0}$ com o produto $[f][g]:=[f . g]$.

O fato da operação $[f][g]$ ser uma operação de grupos segue das propriedades da seção A.1 aplicadas ao caso específico de homotopias de caminhos. Além disso, esta operação está bem definida pois, se $f_{0} \simeq f_{1}$ e $g_{0} \simeq g_{1}$ pelas homotopias $f_{t}$ e $g_{t}$, respectivamente, então $f_{0} . g_{0} \simeq f_{1} . g_{1}$ (concatenação de caminhos) pela homotopia $f_{t} \cdot g_{t}$ (concatenação de homotopias).

Exemplo A.2.1. Seja $X \subset \mathbb{R}^{n}$ um conjunto conexo e $x_{0} \in X$. Então $\pi_{1}\left(X, x_{0}\right)=0$. De fato, quaisquer laços $f_{0}$ e $f_{1}$ no ponto base $x_{0}$ são homotópicos via $f_{t}(s)=(1-t) f_{0}(s)+t f_{1}(s)$.

Se $X$ é conexo por caminhos, $\pi_{1}\left(X, x_{0}\right)$ e $\pi_{1}\left(X, x_{0}\right)$ são isomorfos para todo $x_{0}, x_{1} \in X$. Neste caso, denotamos $\pi_{1}(X)$ sem o ponto base.

Definição A.2.4. Um espaço topológico $X$ é simplesmente conexo se é conexo por caminhos e $\pi_{1}(X)$ é o grupo trivial.

Isto é, um espaço é simplesmente conexo se existe apenas uma classe de homotopia de caminhos ligando dois de seus pontos. 


\section{Homomorfismo induzido}

Denotamos por $\varphi:\left(X, x_{0}\right) \rightarrow\left(Y, y_{0}\right)$ uma função que leva o ponto base $x_{0}$ no ponto base $y_{0}$. Podemos usar $\varphi$ para definir um homomorfismo entre os grupos fundamentais $\pi_{1}\left(X, x_{0}\right)$ e $\pi_{1}\left(Y, y_{0}\right)$. Mais precisamente

$$
\begin{aligned}
& \varphi_{*}: \pi_{1}\left(X, x_{0}\right) \rightarrow \pi_{1}\left(Y, y_{0}\right) \\
& {[f] \quad \mapsto \quad[\varphi(f)] .}
\end{aligned}
$$

$\varphi_{*}$ tem as seguintes propriedades

1. Se $\left(X, x_{0}\right) \stackrel{\psi}{\rightarrow}\left(Y, y_{0}\right) \stackrel{\varphi}{\rightarrow}\left(Z, z_{0}\right)$ então $(\varphi \circ \psi)_{*}=\varphi_{*} \circ \psi_{*} ;$

2. $\left(I d_{X}\right)_{*}=I d_{\pi_{1}\left(X, x_{0}\right)}$.

Estas duas propriedades são o que fazem do grupo fundamental um funtor. Se $\varphi$ é um homeomorfismo com inversa $\psi$, então $\varphi_{*}$ é um isomorfismo com inversa $\psi_{*}$, pois $\varphi_{*} \circ \psi_{*}=(\varphi \circ \psi)_{*}=$ $\left(I d_{X}\right)_{*}=I d_{\pi_{1}\left(X, x_{0}\right)}$.

\section{A.3 Espaços de recobrimento}

Definição A.3.1. Um recobrimento de um espaço topológico $X$ é composto de um espaço de recobrimento $\tilde{X}$ com uma função de recobrimento $p: \tilde{X} \rightarrow X$ tal que existe uma cobertura aberta $\left\{U_{\alpha}\right\}$ de $X$ tal que, para todo $\alpha, p^{-1}\left(U_{\alpha}\right)$ é uma união disjunta de abertos em $\tilde{X}$, cada um deles homeomórfico (por p) a $U_{\alpha}$.

Definição A.3.2. Dado um recobrimento $p: \tilde{X} \rightarrow X$ de $X$, o levantamento de uma função $f: Y \rightarrow X$ é uma função $\tilde{f}: Y \rightarrow \tilde{X}$ tal que $p \tilde{f}=f$.

Ou seja, um levantamento é uma função $\tilde{f}$ tal que o seguinte diagrama comuta:

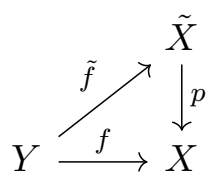

Proposição A.3.1 (Levantamento de homotopias). Dado um recobrimento $p: \tilde{X} \rightarrow X$, uma homotopia $f_{t}: X \rightarrow Y$ e uma função $\tilde{f}_{0}: Y \rightarrow \tilde{X}$ que é um levantamento de $f_{0}$, então existe uma única homotopia $\tilde{f}_{t}: Y \rightarrow \tilde{X}$ de $f_{0}$ que é um levantamento de $f_{1}$.

O teorema a seguir é chamado teorema fundamental de levantamento.

Teorema A.3.1. Dado de recobrimento $p:\left(\tilde{X}, \tilde{x}_{0}\right) \rightarrow\left(X, x_{0}\right)$, uma função $f:\left(Y, y_{0}\right) \rightarrow\left(X, x_{0}\right)$ com $Y$ conexo por caminhos e localmente conexo por caminhos. Então um levantamento $\tilde{f}:\left(Y, y_{0}\right) \rightarrow$ $\left(\tilde{X}, \tilde{x}_{0}\right)$ de $f$ existe se, e somente se, $f_{*}\left(\pi_{1}\left(Y, y_{0}\right)\right) \subset p_{*}\left(\pi_{1}\left(\tilde{X}, \tilde{x}_{0}\right)\right)$.

Uma consequência do teorema fundamental de levantamento é que um recobrimento simplesmente conexo de um espaço $X$ conexo por caminhos e localmente conexo por caminhos é um recobrimento para todo outro recobrimento conexo por caminhos de $X$. Este fato inspira a definição de recobrimento universal.

Definição A.3.3. $O$ recobrimento universal de $X$ é o recobrimento simplesmente conexo de $X$, que é único a menos de isomorfismo.

Por exemplo. o recobrimento universal de $S^{1}$ é $p: \mathbb{R} \rightarrow S^{1} \operatorname{com} p(x)=(\cos (x), \sin (x))$. O recobrimento universal de $S^{2}$ é qualquer automorfismo de $S^{2}$, pois $S^{2}$ é simplesmente conexo. 
Definição A.3.4. Um automorfismo $f: \tilde{X} \rightarrow \tilde{X}$ que respeita a função de recobrimento, isto é, $p \circ f=p$, é chamado de automorfismo de recobrimento ${ }^{2}$. Estes automorfismos formam um grupo, que denotamos por $G(\tilde{X}, X)$.

Um automorfismo de recobrimento leva cada fibra $p^{-1}(x)$ em si mesma.

Proposição A.3.2. Se $\tilde{X}$ é simplesmente conexo e localmente conexo por caminhos, então $G(\tilde{X}, X)$ é isomorfo a $\pi_{1}\left(X, x_{0}\right)$

$\mathrm{O}$ isomorfismo

$$
\begin{array}{ll}
\varphi: \pi_{1}\left(X, x_{0}\right) & \rightarrow G(\tilde{X}, X) \\
\alpha & \mapsto \varphi_{\alpha}: \tilde{X} \rightarrow \tilde{X}
\end{array}
$$

é definido do seguinte modo: escolhemos $\tilde{x}_{0} \in p^{-1}\left(x_{0}\right)$. Dado $\tilde{x} \in \tilde{X}$, ligamos $\tilde{x}$ e $\tilde{x}_{0}$ por um caminho $\tilde{b}$ em $\tilde{X}$. Seja $b=p(\tilde{b}), a \in \alpha$ um ponto do laço $\alpha$ e $x=p(\tilde{x})$. Então $b a b^{-1}$ é um caminho fechado com ponto base $x$.

O levantamento de $b a b^{-1}$ termina num ponto $\tilde{y} \in p^{-1}(x)$. Defino $\varphi_{\alpha}(\tilde{x})=\tilde{y}$.

\section{A.4 Homologia}

Vimos nas seções A.1 e A.2 uma maneira de associar um grupo a um espaço topológico, usando homotopias. No entanto, grupos de homotopia usualmente são difíceis de calcular, embora simples de definir. O caso do grupo fundamental é mais intuitivo, mas grupos gerais de homotopia são difíceis de ser obter para muitos espaços. Por outro lado, os grupos de homologia são difíceis de definir e, após certo trabalho estabelecendo ferramentas e técnicas, bem mais fáceis de calcular. As propriedades de homologia são geralmente usadas, e não sua definição.

Vamos introduzir a ideia de homologia informalmente e depois definir homologia simplicial. A teoria mais geral para espaços topológicos pode ser estendida a partir daí, e, por fim, citaremos uma referência para uma definição de uma teoria de homologia, que é totalmente algébrica.

Uma $n$-célula pode ser pensada como um espaço de dimensão $n$ aberto e limitado. Assim, uma 0-célula é um ponto, uma 1-célula é um arco, uma 2-célula é uma deformação de uma bola aberta, etc. Mais formalmente, se $D^{n}$ o disco de dimensão $n$, uma $n$-célula em um espaço topológico $X$ é um par $\left(E, h_{E}\right)$ tal que $E \subset X, h_{E}: D^{n} \rightarrow X$ é contínua e $h_{E}$ é um homeomorfismo entre o interior de $D_{n}$ e $E$.

Um espaço pode ser construído usando $n$-células, e esta construção é chamada de $C W$-complexo ou complexo simplicial.

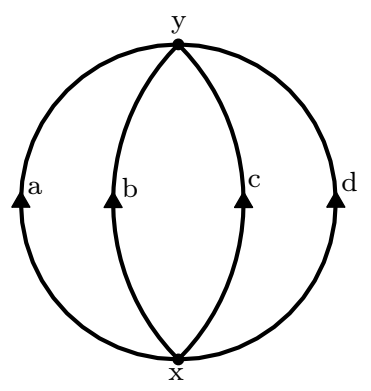

Figura A.1: $X_{1}$.

Os três exemplos a seguir são de [Hat02]. Considere o $C W$ complexo da figura, que é composto por dois pontos $x$ e $y$ ligados por quatro $\operatorname{arcos} a, b, c$ e $d$. Vamos chamá-lo de $X_{1}$. Ao estudar o grupo fundamental, consideramos laços começando e terminando num ponto base. Por exemplo, o laço $a b^{-1}$ começa e termina em $x$. Digamos que para o primeiro grupo de homologia, vamos considerar equivalentes laços que percorrem o mesmo caminho, sem se importar com o ponto base. Assim o laço $a b^{-1}$ e o laço $b^{-1} a$ são iguais. Mais geralmente, o grupo de laços agora é abeliano e os laços são ciclos sem um ponto base fixado.

Mudando para a notação aditiva, que geralmente é usada para grupos abelianos, uma cadeia de arestas é uma combinação linear inteira de $a, b, c$ e $d$. Uma cadeira é um ciclo se ela entra e sai o mesmo número de vezes de um vértice, então $k a+l b+m c+n d$ é um ciclo se $k+l+m+n=0$.

Introduzindo a linguagem de homologia, seja $C_{1}$ o grupo abeliano livre gerado pelas arestas $a, b$, $c$ e $d$, cujos elementos são cadeias de arestas (cadeias de dimensão 1 ), e seja $C_{0}$ o grupo abeliano livre

\footnotetext{
${ }^{2}$ Em inglês, deck transformation.
} 
gerado pelos vértices $x$ e $y$, cujos elementos são combinações lineares de $x$ e $y$ (cadeias de dimensão $0)$. Definimos um homomorfismo $\partial: C_{1} \rightarrow C_{0}$ que manda cada elemento $a, b, c$ e $d$ em $y-x$, que é o vértice em que termina cada aresta menos o vértice em que começa. Os ciclos pertencem ao núcleo de $\partial$, pois $\partial(k a+l b+m c+n d)=(k+l+m+n) y-(k+l+m+n) x . a-b, a-c, c-d \in C_{1}$ formam uma base para $\operatorname{ker}(\partial)$ e este fato pode ser interpretado geometricamente como a existência de três "furos" em $X_{1}$.

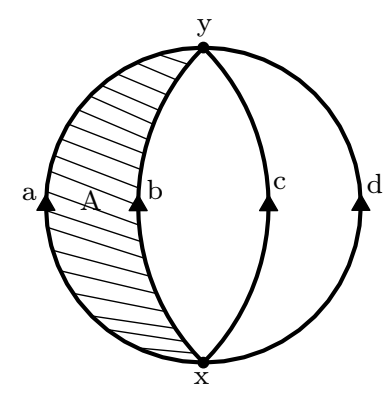

Figura A.2: $X_{2}$.

Vamos adicionar agora a 2-célula $A$ nesta construção entre o ciclo $a-b$, formando o CW-complexo $X_{2}$. Se $A$ for orientada no sentido horário, sua fronteira é o ciclo $a-b$. Este ciclo agora é homotópico a 0 , já que a superfície de $A$ permite que ele seja contraído até um ponto, isto é, o "furo" em torno do qual estava $a-b$ deixa de existir.

Voltando para o formalismo do exemplo anterior, podemos definir agora dois homomorfismos $C_{2} \stackrel{\partial_{2}}{\longrightarrow} C_{1} \stackrel{\partial_{1}}{\longrightarrow} C_{0}$, onde $C_{2}$ é o grupo cíclico infinito gerado por $A$ e $\partial_{2}(A)=a-b$. $\partial_{1}$ é o mesmo homomorfismo do exemplo $X_{1}$. Considere o quociente $\operatorname{ker}\left(\partial_{1}\right) / \operatorname{Im}\left(\partial_{2}\right)$, os ciclos de uma dimensão 1 (gerados por $a-b$, $a-c$ e $c-d$ ) módulo $a-b$. Este quociente é o grupo de homologia $H_{1}\left(X_{2}\right)$, que é um grupo livre abeliano com 2 geradores. No exemplo $X_{1}$ tínhamos $C_{2}=\{0\}$ um grupo trivial pois não havia nenhuma 2-célula, logo $H_{1}\left(X_{1}\right)=$ $\operatorname{ker}\left(\partial_{1}\right) / \operatorname{Im}\left(\partial_{2}\right)=\operatorname{ker}\left(\partial_{1}\right) \cong \mathbb{Z}^{3}$ é um grupo livre abeliano com 3 geradores. Isto é, antes haviam 3 "furos" no espaço e agora há 2 e $H_{1}\left(X_{2}\right) \cong \mathbb{Z}^{2}$.

Se adicionarmos uma nova 2-célula $B$ nesta construção entre

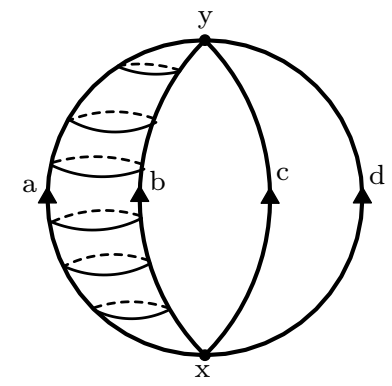

Figura A.3: $X_{3}$. o ciclo $a-b$, temos um novo CW-complexo $X_{3}$. O grupo $C_{2}$ de cadeias de dimensão 2 agora é o grupo das combinações inteiras de $A$ e $B$, e o homomorfismo de fronteira $\partial_{2}: C_{2} \rightarrow C_{1}$ manda tanto $A$ quanto $B$ para $a-b$. O grupo de homologia $H_{1}\left(X_{3}\right)=$ $\operatorname{ker}\left(\partial_{1}\right) / \operatorname{Im}\left(\partial_{2}\right)$ é o mesmo de $X_{2}$. No entanto, agora $\operatorname{ker}\left(\partial_{2}\right)$ não é mais trivial, e sim o grupo cíclico infinito gerado por $A-B$. Logo, pensando que $C_{3}$ é trivial, $H_{2}\left(X_{3}\right)=\operatorname{ker}\left(\partial_{2}\right) \cong \mathbb{Z}$.

O ciclo $A-B$ é um ciclo de dimensão 2 , que topologicamente é uma esfera gerada por $A, B$ e sua fronteira $a-b$. Assim, $H_{1}$ não "detecta" nenhum "furo" em $a-b$, mas $H_{2}$ detecta um "furo" que é o interior de $A-B$.

O n-ésimo grupo de homologia é definido por $H_{n}(X)=$ $\operatorname{ker}\left(\partial_{n}\right) / \operatorname{Im}\left(\partial_{n+1}\right)$. Intuitivamente, os elementos de $H_{1}(X)$ são curvas que contornam "buracos" de dimensão $1 \mathrm{em} X$. Para $n$ grande, esta intuição se perde e fica difícil definir $\partial_{n}$. Para contornar esta dificuldade, dividimos poliedros em simplexos, e daí surge a homologia simplicial, que é aplicável a CW-complexos divididos em simplexos.

Um $n$-simplexo é o fecho convexo de $n+1$ pontos em $R^{n}, n$ linearmente independentes e 0 . Um 2 simplexo é um triângulo plano e um exemplo de 3 -simplexo é o fecho convexo de $\left\{0, e_{1}, e_{2}, e_{3}\right\}$.

A classe de espaços de CW-complexos é restritiva. Para um teoria de homologia para espaços topológicos quaisquer, usamos funções contínuas entre simplexos e o espaço topológico em questão. Esta abordagem dá origem a homologia singular.

As teorias de homologia simplicial e singular satisfazem algumas propriedades algébricas. Estas propriedades inspiraram uma definição axiomática e algébrica teoria de homologia. Esta definição pode ser consultada na página 183 de [Bre93] ou o capítulo 1 de [Lim12].

A seguir, caracterizamos o primeiro grupo de homologia para espaços conexos por caminho.

Proposição A.4.1. Se $X$ é um espaço topológico conexo por caminhos, então a abelianização de $\pi_{1}(X)$ é isomorfa a $H_{1}(X)$.

Para a demonstração da proposição acima, veja a seção 2.A de [Hat02]. 


\section{Referências Bibliográficas}

[Bre93] Glen E Bredon. Topology and geometry, volume 139. Springer-Verlag, 1993. 71, 72, 76

[Gut03] Eugene Gutkin. Billiard dynamics: a survey with the emphasis on open problems. Regular and chaotic dynamics, 8(1):1-13, 2003. 2, 3

[Hat02] Allen Hatcher. Algebraic topology. Disponível em: https://www.math.cornell.edu/ พhatcher/AT/ATpage.html, 2002. 71, 75, 76

[HH00] Lorenz Halbeisen e Norbert Hungerbühler. On periodic billiard trajectories in obtuse triangles. SIAM review, 42(4):657-670, 2000. 3, 9, 22, 34

[Hol93] Fred Holt. Periodic reflecting paths in right triangles. Geometriae Dedicata, 46(1):73-90, 1993. 11

[Hoo07] W Patrick Hooper. Periodic billiard paths in right triangles are unstable. Geometriae Dedicata, 125(1):39-46, 2007. 3

[HS09] W Patrick Hooper e Richard Evan Schwartz. Billiards in nearly isosceles triangles. Journal of Modern Dynamics, 3(2):159-231, 2009. 3, 33, 39, 45, 49, 56, 57, 58, 59, 61, 68

[HS14] W Patrick Hooper e Richard Evan Schwartz. Erratum: Billiards in nearly isosceles triangles. Journal of Modern Dynamics, 8(1):133-137, 2014.

[KH97] Anatole Katok e Boris Hasselblatt. Introduction to the modern theory of dynamical systems, volume 54. Cambridge university press, 1997.

[Lim06] Elon Lages Lima. Grupo fundamental e espaços de recobrimento. Instituto de Matemática Pura e Aplicada. 3a edição., 2006. 71

[Lim12] Elon Lages Lima. Homologia básica. Instituto de Matemática Pura e Aplicada. 2a edição., 2012. 71,76

[Mas86] Howard Masur. Closed trajectories for quadratic differentials with an application to billiards. Duke Math. J, 53(2):307-314, 1986. 7

[MKS66] Wilhelm Magnus, Abraham Karrass e Donald Solitar. Combinatorial Group Theory: Presentations of Groups in Terms of Generator and Relations. Interscience publishers, 1966. 64

[ML13] Saunders Mac Lane. Categories for the working mathematician, volume 5. Springer Science \& Business Media, 2013. 71

[MT02] Howard Masur e Serge Tabachnikov. Rational billiards and flat structures. Handbook of dynamical systems, 1:1015-1089, 2002. 3, 65, 68, 70

[Mun84] James R Munkres. Elements of algebraic topology, volume 2. Addison-Wesley Menlo Park, 1984. 71

[Mun00] James R Munkres. Topology. Prentice Hall, 2000. 71 
[O'n06] Barrett O'neill. Elementary differential geometry. Academic press, 2006.

[Sch06] Richard Evan Schwartz. Obtuse triangular billiards i: near the $(2,3,6)$ triangle. Experimental Mathematics, 15(2):161-182, 2006. 28, 29

[Sch09] Richard Evan Schwartz. Obtuse triangular billiards ii: One hundred degrees worth of periodic trajectories. Experimental Mathematics, 18(2):137-171, 2009. 27, 28, 29

[Spi99] Michael Spivak. A Comprehensive Introduction to Differential Geometry, Vol. 1. Publish or Perish; 3rd edition, 1999.

[Tab95] Serge Tabachnikov. Billiards. Société mathématique de France, 1995. 3

[Tab05] Serge Tabachnikov. Geometry and billiards, volume 30. American Mathematical Soc., 2005. 3

[Thu14] William P Thurston. Three-Dimensional Geometry and Topology, Volume 1, volume 1. Princeton university press, 2014. 63, 64

[Vee89] William A Veech. Teichmüller curves in moduli space, eisenstein series and an application to triangular billiards. Inventiones mathematicae, 97(3):553-583, 1989. 33

[VGS92] Yaroslav Bogdanovich Vorobets, Gregorii Aleksandrovich Gal'perin e Anatolii Mikhailovich Stepin. Periodic billiard trajectories in polygons: generating mechanisms. Russian Mathematical Surveys, 47(3):5-80, 1992. 3, 7, 11, 15, 19, 27 


\section{Índice Remissivo}

3-espinha, 34

$O(\omega)$, veja ladrilho de órbita

$U(\omega, T)$, veja desdobramento

$k$-leque, 34

$n$-célula, 75

$n$-simplexo, 76

órbita bilhar, 1

órbita estável, 14

órbita instável, 14

órbita reticulada hexagonal, 39

órbita reticulada quadrada, 40

órbitas perpendiculares, 7

automorfismo de recobrimento, 75

bloco, 54

CW-complexo, 75

desdobramento, 5,14

direção completamente periódica, 68

dobradiças, 53

espaço dos parâmetros, 13

feixe de órbitas paralelas, 5

fluxo bilhar, 1

função comparadora, 43

grupo de homologia, 76

grupo de rotação, 6

grupo de Veech, 68

grupo diedral, 7

grupo fundamental, 73

holonomia, 63

homotopia, 71

homotopia de caminhos, 73

ladrilho de órbita, 16

levantamento, 74

métrica Hausdorff, 52

palavra estável, 14

palavra finita, 14 palavra infinita, 14

polígono irracional, 6

polígono racional, 6

recobrimento, 74

recobrimento universal, 74

região pivô, 56

superfície de translação, 65

superfície de Veech, 68

superfícies de Veech, 65

transformação bilhar, 1

triângulo de Fagnano, 8

triângulo de Veech, 33

vértices base, 34

vértices inferiores, 34

vértices ponta, 34

vértices superiores, 34 